\title{
ANALISIS DE LA ESTRUCTURA DE ESPECTROS DE ABSORCION Y DISPERSION PRODUCIDOS EN PRESENCIA DE EFECTOS ENSANCHADORES DE ORIGEN ALEATORIO
}

TESIS QUE PRESENTA

\author{
HOMERO JIMENEZ DOMINGUEZ
}

PARA OBTENER EL GRADO DE

DOCTOR EN CIENCIAS

\author{
UNIVERSIDAD AUTONOMA METROPOLITANA \\ DIVISION DE CIENCIAS BASICAS E INGENIERIA
}

OCTUBRE DE 1997 
Esta tesis fue dirigida por el Dr. Salvador Cruz Jiménez a quien agradezco sus indicaciones y agudos comentarios que enriquecieron el trabajo, asi como su paciencia y el empeño puestos en mi graduación.

Doy las gracias a los Drs. Manuel Corona Galindo, Luis González Tovany, Julio Herrera Velázquez y Antonio Muñoz Flores quienes participaron en la revisión de este trabajo. Además reconozco la gran ayuda que me brindaron los Drs. Luis González Tovany y Mayo Villagrán Muñiz por haberme proporcionado varias referencias que fueron relevantes para la elaboración de esta tesis. Agradezco al Dr. Enrique Camps Carvajal por haberme permitido el acceso a su registro de líneas espectrales, provenientes de un plasma de helio producido por microondas en un dispositivo construido por él en el ININ. Al Ing. Victor Hernández Magadán debo el arreglo del formato final del texto.

Agradezco al Instituto Nacional de Investigaciones Nucleares el haberme otorgado facilidades para la realización de esta tesis.

Dedico este trabajo a mi madre, a mi esposa y a mi hijo. 


\section{INDICE}

\section{Pág.}

RESUMEN

CAPITULO I

1. Convolución 11

2. El perfil de Voigt 13

3. La función de dispersión del plasma (FDP) 14

CAPITULO ॥ 17

EL PERFIL DE VOIGT: SU RELACION CON LA FUNCION 17

DE ERROR COMPLEJA Y CON LA FUNCION DE DISPERSION DEL PLASMA

1. Introducción

2. El perfil de Voigt como la convolución de la función lorentziana con la distribución gaussiana

3. Relación del perfil de Voigt con la función de error 25 compleja

4. Relación del perfil de Voigt con la función de dispersión del plasma [21]

5. Un método para estimar temperatura y densidad

38 electrónicas en un plasma 
2. Funciones causales

3. Relaciones de dispersión y relaciones de Kramers-Krönig

4. El oscilador armónico

a). Oscilaciones libres 53

b). Oscilaciones armónicamente forzadas $\quad 54$

c). La solución generalizada $\quad 55$

d). Analiticidad y Causalidad 57

5. Dos maneras alternativas y sencillas para obtener 60 las relaciones de Kramer-Krönig

5.1 Uso de la función escalón $\quad 60$

5.2 Uso de la función signum $\quad 64$

6. Primer ejemplo: Forma aproximada de la FDP 68 propuesta por B. D. Fried et al. [30]

7. Segundo ejemplo: Forma aproximada de la FDP 70 propuesta por Martín y Gonzalez [31]

8. Tercer ejemplo: Forma aproximada de la FDP propuesta por Robinson and Newman [32]

9. Cuarto ejemplo: Forma aproximada de la FDP propuesta en este trabajo

1. Introducción

2. Conexión entre los perfiles $H_{A P R}(v, a)$, $D_{A P R}(u, a)$ y la función de dispersión del plasma

3. Generalización de los perfiles espectroscópicos 80 $H_{A P R}(u, a)$ y $D_{A P R}(u, a)$ para incluir un parámetro de asimetría 
4. Ejemplo de aplicación a un caso experimental para $\mathbf{8 6}$ probar el modelo asimétrico de absorción propuesto

5. Descripción del problema 87

5.1 Los parámetros de la descarga $\quad 87$

5.2 El instrumento de observación 88

5.3 Análisis del espectro registrado 89

5.4 Cálculo de las temperaturas 91

5.5 Análisis de los resultados 92

CAPITULO V 97

UN NUEVO METODO SIMPLE PARA ANALIZAR 97

VOIGTIANAS DE PEQUEÑO CONTENIDO GAUSSIANO CON AYUDA DE DATOS DISPA

1. Introducción 97

2. Fundamentos del método 99

3. Aplicación del método 103

3.1 Aplicación a una línea hipotética 103

3.2 Criterio para elegir $v_{\max }$ de los datos DISPA 105

3.3 Aplicación del método a una línea 107 experimental real

4. Resultados y discusión

CAPITULO VI

USO DE LA FUNCION DE DISPERSION DEL PLASMA

PARA PROPONER UN MODELO DE LINEA ASIMETRICO $Y$ PARA DESCRIBIR LA SUSCEPTIBILIDAD MAGNETICA COMPLEJA

1. Introducción

2. Relación entre susceptibilidad magnética compleja 117 y la función de dispersión del plasma 
3. Una generalización sencilla de la función de dispersión del plasma para incluir un parámetro de asimetría

4. Aplicación del modelo a una línea experimental 


\section{RESUMEN}

En este trabajo se muestra la utilidad de la Función de Dispersión del Plasma (FDP) en el campo de las espectroscopías (óptica, UV, Mössbauer, RMN, RPE, etc). En particular se proponen nuevos modelos de formas de lineas espectrales.

En virtud de que la FDP ha sido usada solamente en el ámbito de la física de plasmas para realizar cálculos numéricos en propagación de ondas, el parentesco del perfil de absorción de Voigt con la FDP no ha sido explicitamente destacado en la literatura. Es a partir de este parentesco que se sugiere la idea de aplicar la FDP en espectroscopia, ya que además aquí se demuestra que su parte real corresponde a los perfiles de dispersión espectroscópicos.

Antes de hacer aplicaciones a la espectroscopia en este trabajo, se propone un nuevo método para estimar temperatura y densidad electrónicas en un plasma por medio de la deconvolución de la parte imaginaria de la FDP, considerando que la amplitud de la onda que se propaga en el plasma decae con el parámetro de amortiguamiento de Landau.

Posteriormente, reconociendo que la FDP es una relación de dispersión y que sus partes real e imaginaria están vinculadas por medio de la transformada de Hilbert, se obtiene un nuevo par absorción-dispersión aproximado para su aplicación a líneas espectrales de absorción y dispersión, en el caso de pequeña contribución gaussiana. A continuación este par absorción-dispersión se generaliza incluyendo en él un factor de asimetría que permite analizar lineas espectrales asimétricas de absorción y 
dispersión que se presentan con mucha frecuencia en espectroscopia. Este modelo se aplica aquí para estimar la temperatura de la componente neutra de un plasma de helio producido por resonancia ciclotrónica de electrones. El valor de la temperatura que se obtiene con este modelo de linea, es congruente con los reportados en la literatura para este tipo de plasmas.

A continuación se desarrolla un nuevo método para deconvolucionar líneas espectrales simétricas caracterizado por no emplear técnicas numéricas de ajuste. El método se basa en el uso adecuado del valor máximo del diagrama DISPA (DISPersión-Absorción) teórico y los datos DISPA para una linea espectral experimental. Como ejemplo de aplicación del método se realiza la deconvolución de una línea RPE experimental de una solución de potasio en amonio tomada de la literatura. Esta línea fue elegida por su bajo contenido gaussiano y por tener un grado de asimetría muy pequeño.

Finalmente la función de dispersión del plasma exacta se somete a un proceso de asimetrización que produce expresiones elegantes para las partes real e imaginaria de la función de dispersión del plasma asimetrizada. Asimismo, se demuestra que la susceptibilidad magnética compleja puede escribirse en términos de la función de dispersión del plasma tanto en su forma común como en su forma asimetrizada, generalizando asi la solución de las ecuaciones de Bloch. La FDP asimetrizada constituye un nuevo modelo de perfil de línea. Por otra parte, se encuentra una importante relación de carácter general, entre los parámetros relevantes de la línea. De esta relación se puede deducir que el grado de asimetría inducido sobre el perfil por factores no especificados, no puede ser mayor a una cantidad proporcional a su ensanchamiento gaussiano so pena de obstruir toda información espectral. Es 
importante hacer notar que este concepto era conocido intuitivamente en espectroscopia y aqui se confirma formalmente. Como aplicación de este nuevo modelo de línea se realiza nuevamente la deconvolución de la linea RPE de potasio-amonio produciendo resultados congruentes con los obtenidos antes en esta tesis. 


\section{INTRODUCCION GENERAL}

Posiblemente sea apropiado decir que la espectroscopía es la ventana indiscreta a través de la cual el hombre observa las intimidades del universo. Cada parte de éste, independientemente de sus dimensiones, se encuentra en constante interacción con las demás por medio de diferentes mecanismos. El resultado de esa interacción es la emisión de una señal que puede ser detectada por algún observador. Lo importante de esa señal es que porta información acerca de los mecanismos involucrados en la interacción y de las condiciones bajo las cuales se lleva a cabo ésta, asi como de las características de los sistemas interactuantes. La espectroscopía surge como una actividad vía la cual se extrae la información que porta la señal, estudiando la distribución de la intensidad de esta señal mediante el análisis de un observable que la caracteriza como por ejemplo la longitud de onda, la frecuencia, la energía o el número de onda. En efecto, la espectroscopía ha sido el medio por el cual los científicos han adquirido la mayor parte del conocimiento que se tiene acerca de la materia y de su interacción con la energia. En su libro, "Principles of Modern Physics" [1], R. B. Leighton dice que "de todas las herramientas que se han aplicado al estudio de la estructura detallada de la materia, puede decirse que la espectroscopia ha sido más aplicada a más problemas de más maneras, y ha producido más información fundamental que ninguna otra". Originalmente la espectroscopía estaba limitada al análisis de señales de luz visible, pero nuevas maneras de generar y detectar otras formas de energía han ampliado considerablemente su campo de aplicación al estudiar señales en todo el espectro electromagnético. Incluso, el término "espectroscopia" ha sido aplicado en otros campos en los que la radiación electromagnética no está involucrada; ejemplo de ésto es la espectroscopía de masas. Es más por razones históricas que por definición, el hecho de que a esta técnica se le llame ocasionalmente espectroscopía. En efecto, las placas sensibilizadas que obtenia Aston durante sus experimentos para determinar masas de isótopos en 1919, se parecian mucho a las 
placas de un espectro óptico de los obtenidos en absorción atómica. Sin embargo, en una espectroscopía "verdadera" la intensidad de una señal tiene, en general, una distribución contínua aún en el caso de lineas espectrales, ya que éstas tienen siempre una anchura natural diferente de cero debido al principio de incertidumbre. La firma espectral de una señal se puede describir en términos de una función de la energía, de la frecuencia o de la longitud de onda, las cuales pueden ser tratadas como variables continuas, en tanto que las masas atómicas y moleculares son siempre cantidades discretas, determinando por lo tanto un patrón discreto de líneas sin estructura al cual se le denomina "espectro de masas".

La información provista por la señal se encuentra contenida en la estructura de ésta, en virtud de una operación natural que puede ser descrita matemáticamente por una integral de convolución. Si, por ejemplo, a la radiación emitida por un átomo fijo y aislado se le llama señal portadora, la información acarreada podría ser el efecto que sobre la portadora tendría el hecho de que los átomos emisores no estuvieran fijos y aislados sino moviéndose caóticamente. En general, el efecto consiste en el ensanchamiento de la distribución de frecuencias de la señal portadora. Es necesario enfatizar que el efecto se produce no por modulación sino por convolución. De acuerdo con lo dicho se sigue que el concepto de convolución no es solamente matemático sino también físico y está omnipresente en las observaciones en el siguiente sentido: para estudiar cualquier fenómeno es necesario hacer uso de un instrumento de observación el cual necesariamente actúa sobre la señal observada, de tal manera que ésta sufre un ensanchamiento adicional al que ya acarreaba.

Hay algo más, sin embargo, que debe decirse con respecto a la señal que se está observando: siendo ésta un ente físico, debe obedecer al principio de causalidad. Por tanto, debe procurarse que los modelos que se usen para simular su forma, sean modelos causales. En la literatura se encuentran modelos empiricos útiles que no parten de ese principio y que son, por lo tanto, limitados. Como se verá en este trabajo, 
en un modelo causal el espectro de frecuencias de la señal está compuesto de dos partes ligadas intimamente y que la describen completamente, a saber, una parte de absorción y otra de dispersión. Es papel del espectroscopista analizar la estructura de la señal para extraer la información contenida en ella. Esta clase de tareas comenzó a ser realizada desde principios de este siglo por los astrónomos para "medir" la temperatura superficial de las estrellas y posteriormente, para "medir" la velocidad de expansión del universo a través del corrimiento hacia el rojo o hacia el azul de la luz emitida por las estrellas y galaxias lejanas. Esas tareas se ampliaron al aparecer nuevas espectroscopías cuyos principios son comunes a todas ellas: espectroscopias de rayos X, ultravioleta, infrarroja, Raman, de fluorescencia, de resonancia paramagnética electrónica (RPE), de resonancia magnética nuclear (RMN), Mössbauer, etc.

En esta tesis se presentan nuevos resultados y contribuciones interesantes que ayudan a realizar un análisis de espectros de señales producidas en presencia de dos tipos de mecanismos que modifican el perfil de las líneas espectrales, a saber: mecanismos ensanchadores de origen estocástico y mecanismos asimetrizadores; por ejemplo, en RPE un mecanismo ensanchador de líneas es la distribución azarosa de ejes cristalinos en una muestra en polvo, mientras que en espectroscopía óptica un mecanismo ensanchador es el movimiento térmico de los átomos emisores.

Esta tesis ha sido elaborada tomando como base a la función de dispersión del plasma. En esta función están contenidos implícitamente conceptos que sirven de apoyo a la estructura del trabajo, i. e. los conceptos de convolución, perfil de Voigt y relaciones de Kramers-Krönig. Trabajando con estos conceptos y algunas sugerencias de este autor, se ha logrado extender el campo de aplicación de la FDP mediante la propuesta de nuevos perfiles espectrales de absorción y dispersión basados en ella, 
asi como usar esa función para representar la susceptibilidad magnética compleja cuando el sistema magnético que se considere sea perturbado por mecanismos aleatorios modificadores de línea.

En el capítulo I se describen brevemente el concepto de convolución en el campo de la ingeniería, el perfil de Voigt y la FDP.

En el capitulo Il se presenta la manera de obtener el perfil de Voigt a partir de la convolución del perfil de Lorentz con la distribución gaussiana. Para ésto, se trabaja con una descripción en términos de una variable y de un parámetro adimensionales, que contienen toda la información que caracteriza tanto al perfil de Lorentz como a la gaussiana y facilitan el uso de escalas arbitrarias, útiles para propósitos de ajuste de líneas experimentales. Enseguida se enfatiza la relación del perfil de Voigt con la función de error compleja. En este contexto, se resalta la relación del perfil de Voigt con la función de dispersión del plasma. Como esta función es la piedra angular en el desarrollo de este trabajo, se procede detalladamente a su obtención, a la manera en que se hace en física de plasmas. En este proceso, se observa que el perfil de Voigt es proporcional a la parte imaginaria de la FDP. Al final del capitulo II, se propone un nuevo método para estimar la temperatura y la densidad electrónicas en un plasma. El método se basa en la deconvolución de la parte imaginaria de la FDP y fué publicado en la Ref. [6].

En el capítulo III se presta atención a las expresiones aproximadas de los perfiles de absorción y dispersión de líneas espectrales que pueden ser tratadas con el perfil de Voigt. Estas expresiones surgen del manejo de este perfil cuando tiene un contenido mucho mayor de gaussiana que de lorentziana o viceversa. En ocasiones el espectroscopista sabe a priori que su espectro contiene más contribución de un tipo que del otro y entonces, para simplificar cálculos analíticos y ahorrar tiempo, puede hacer uso de las expresiones aproximadas. Por éso las expresiones aproximadas de 
la FDP que existen en la literatura son de utilidad. Sin embargo es necesario asegurarse de que las formas aproximadas que se usen o propongan cumplan con las propiedades de simetría de la FDP; en otras palabras, que satisfagan las relaciones de Kramers-Krönig. En este capítulo se estudian tres ejemplos de formas aproximadas de la FDP, tomados de la literatura, a fin de revisar si ellas cumplen con las relaciones de Kramers-Krönig las cuales son obtenidas con detalle por medio de un procedimiento alternativo sencillo. Finalmente, se propone una nueva pareja de perfiles absorción-dispersión basada en una expresión aproximada del perfil de Voigt publicada por otros autores y nosotros. Esta pareja será tema del capítulo IV.

En el capítulo IV se presenta la obtención de un nuevo modelo de absorción-dispersión con asimetría para el caso de una pequeña contribución gaussiana. Para ese fin, sirve como punto de partida el par absorción-dispersión obtenido en el capítulo III, incluyendo aqui un factor de asimetría para simular líneas espectrales asimétricas de aparición frecuente en espectroscopia. El factor de asimetría se asocia inmediatamente al perfil de absorción para obtener un perfil asimétrico, del cual se obtiene la transformada de Hilbert para llegar al perfil de dispersión asimétrico. Al final de este capitulo se presenta un ejemplo de aplicación del perfil asimétrico de absorción para obtener la temperatura de la componente neutra de un plasma de helio producido por resonancia ciclotrónica de electrones. Los resultados obtenidos para la temperatura son consistentes con los obtenidos en la literatura para plasmas de este tipo. Una parte del trabajo realizada con esta técnica durante el desarrollo de la presente tesis, fue publicado en la Ref [7].

En el capitulo $V$ se continúa explotando el par simétrico absorción-dispersión y se usa el concepto DISPA (DISPersión vs Absorción) para analizar perfiles voigtianos con pequeño contenido gaussiano. En este capítulo se presenta la obtención de un nuevo método para analizar dichos perfiles, con ayuda de datos DISPA obtenidos de 
lineas experimentales. El método es muy sencillo y se caracteriza por no emplear técnicas numéricas de ajuste para hacer la deconvolución de ese tipo de perfiles. El método es útil para analizar líneas aisladas no traslapadas en cualquier espectroscopia, aún en aquellas en las que experimentalmente no puede obtenerse el perfil de dispersión (por ejemplo espectroscopia Mössbauer). En estos casos este perfil se obtiene tomando la transformada de Hilbert del conjunto de datos experimentales de la línea de absorción. La colección de datos de absorción y dispersión componen los datos DISPA con los que se pueden construir las gráficas DISPA correspondientes a la línea espectroscópica experimental considerada. Como ejemplo de aplicación del método, se calculan las contribuciones lorentziana y gaussiana en la línea experimental RPE de una solución de potasio en amonio reportada por otros autores en la literatura. La elección de esta línea se debió a que, siendo conocidos su pequeña contribución gaussiana, su forma bien definida y su muy pequeño grado de asimetría lel modelo presentado en este capítulo es simétricol, la convierten en un buen sujeto para probar el presente método. Un artículo basado en el método presentado en este capítulo apareció publicado en la Ref. [8].

En el capitulo VI se presenta la obtención de un nuevo resultado que consiste de la expresión asimetrizada y sin aproximaciones de la FDP, así como de la susceptibilidad magnética compleja escrita en términos de esa expresión asimetrizada de la FDP. Con esta forma de la susceptibilidad magnética se describe el comportamiento de un sistema de espines cuando la excitación resonante de los estados de espín por absorción de microondas (espectroscopía RPE) o de radiofrecuencia (espectroscopia $R M N$ ), se produce en presencia de mecanismos ensanchadores de línea, de origen estocástico, así como de mecanismos asimetrizadores. Este tipo de fenómenos no esta considerado en las ecuaciones de Bloch, las cuales producen una forma puramente lorentziana para la susceptibilidad. 
Sin embargo, lo relevante en este capítulo es que la expresión asimetrizada de la FDP constituye un nuevo modelo útil en cualquier espectroscopía, que permite analizar formas de línea voigtianas con asimetría del lado derecho o izquierdo. En este capitulo se resalta la simplicidad con la que una función tan complicada como la FDP permite ser asimetrizada como se hizo aquí. Un aspecto muy importante encontrado en el desarrollo de este capítulo es que el grado de asimetria inducido en el perfil de la línea por factores no especificados, no puede exceder su anchura gaussiana dividida por $\Gamma_{L} \sqrt{\ln } 2$. sin obstruir toda información espectral. La bondad del modelo se compara con la de uno reportado por otro autor en la literatura, encontrándose que es mejor el ajuste con este modelo para la línea que se consideró. Un articulo basado en el modelo presentado en este capítulo, aparecerá publicado en 1997, [63].

Finalmente se presentan las conclusiones generales de este trabajo asi como un apéndice en el que se dan detalles del cálculo conducente a la obtención de la función de dispersión del plasma. 


\section{CAPITULO I}

\section{CONCEPTOS BASICOS}

En este capítulo se describirán brevemente algunos conceptos en los que se basa este trabajo, los cuales serán manejados con mayor detalle y profundidad en capítulos subsecuentes.

\section{1.- CONVOLUCION}

El concepto de convolución se utiliza ampliamente en ingeniería v ciencia. En el capítulo II se presenta en una forma general este concepto, así como la manera en que se aplica para los fines de este trabajo. No obstante, en este capitulo se presentará una forma clara y descriptiva de cómo aparecen en ingeniería el concepto y la expresión matemática de convolución [2]. En la Figura 1-1 se representa esquemáticamente un sistema lineal e invariante en el tiempo, caracterizado por un operador de transformación $T_{\imath} l \mathrm{l}$.

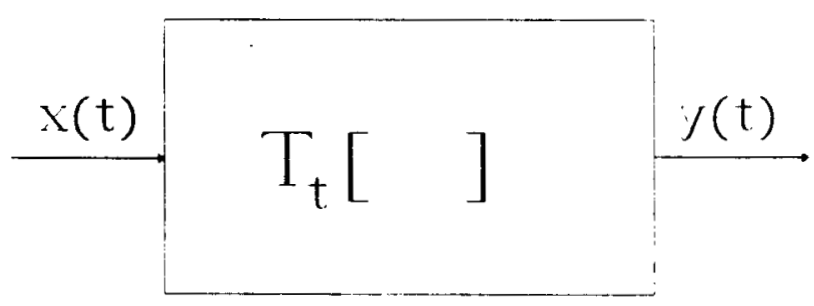

Figura 1-1.- Representación de un sistema lineal e invariante en el tiempo, caracterizado por un operador de transformación $T_{\imath}[]$. 
Es necesario hacer notar que el operador $T_{l}[$ ] actúa solamente sobre funciones de la variable del tiempo $t$, importante aspecto que no se considera en la Ref. [2]. A este sistema llega una señal de entrada $x(t)$ y entrega una señal de salida $y(t)$. La señal de salida se puede escribir simbólicamente como

$$
y(t)=T_{d}[x(t)] .
$$

Sin perder generalidad, cualquier señal de entrada $f(t)$ puede ser representada así:

$$
f(l)=\int_{-\infty}^{\infty} f(T) \delta(l-T) d \tau .
$$

de modo que la señal de entrada.$(t)$ se puede también escribir

$$
x(t)=\int_{-\infty}^{\infty} x(\tau) \delta(t-\tau) d \tau .
$$

Al substituir (1-3) en (1-1), la señal de salida adquiere la forma

$$
\gamma(t)=T_{t}\left[\int_{-\infty}^{\infty} x(\tau) \delta(t-\tau) d \tau\right] .
$$

En vista de que el operador $T_{l}$ [ ] ]actúa solamente sobre funciones de la variable $t$, esta expresión se puede reescribir

$$
y(t)=\int_{-\infty}^{\infty} v(\tau) T_{1}[\delta(t-\tau)] d \tau .
$$

La expresión $T_{\imath}[\delta(\ell-1)\rfloor$ en el integrando de (I-5) representa, de acuerdo con la definición (I-1), la salida o respuesta del sistema a una señal de entrada $x(t)$ con la forma de una delta de Dirac. Llamando $h(t-\tau)$ a esta señal de salida, la expresión (1-5) quedaría ahora escrita como

$$
y(t)=\int_{-\infty}^{\infty} x(\tau) h(t-\tau) d \tau .
$$


La manera en que las funciones.$(\ell)$ y $h(l)$ están consideradas en el integrando de la expresión (I-6) corresponde a la usual en la definición matemática de convolución, como se verá en el siguiente capitulo. La expresión indica que la respuesta o salida de un sistema lineal e invariante en el tiempo, está representada por la convolución de la señal de entrada con la respuesta del sistema a una señal impulsiva de magnitud unitaria pero recorrida hacia la derecha en el tiempo por una cantidad $\tau$.

La expresión (I-6), llamada integral de convolución de las funciones.$l(t)$ y $h(t)$, se acostumbra escribir simbólicamente como:

$$
y(t)=x(t) * h(t) .
$$

Así pues, el procedimiento que condujo a la expresión (1-6) puede ser resumido asi:

1.- Descomponer la señal de entrada a un sistema lineal e invariante en el tiempo, en una suma infinita de pulsos $\delta(t-\tau)$ de magnitud $x(\tau)$.

2.- Determinar la respuesta $h(l)$ del sistema, a una señal impulsiva unitaria $\delta(t)$.

3.- Determinar la respuesta del sistema a cada uno de los pulsos que representan a la señal de entrada.

4.- Superponer las respuestas individuales del sistema a todos los pulsos que representan la señal de entrada, para así obtener la señal de salida del sistema.

\section{2.- EL PERFIL DE VOIGT}

Matemáticamente el perfil de Voigt [3] es la convolución de un perfil de Lorentz con la distribución gaussiana. El perfil de Lorentz describe la forma de línea de una 
señal emitida por un sistema cuántico aislado, cuando éste decae de un nivel excitado a un estado de menor energía. Por su parte la distribución gaussiana representa la manera en la que una cantidad física asociada al sistema bajo estudio se distribuye cuando asume valores al azar (como por ejemplo la velocidad con que aleatoriamente se mueven los átomos emisores en un gas calientel. La expresión matemática del perfil de Voigt es muy útil para describir la forma de las lineas espectrales experimentales ensanchadas por mecanismos externos de naturaleza aleatoria ya que puede ser adaptado con buena aproximación a la forma de dichas líneas cuando no están presentes otros mecanismos ensanchadores tales como aquéllos debidos a la presión. La razón de la utilidad de este perfil reside en que muchas líneas espectrales experimentales pueden ser consideradas, con buena aproximación, como la convolución de un perfil de Lorentz con la distribución gaussiana. El perfil de Voigt se conoce desde principios de siglo y fué usado por los astrónomos para estimar la temperatura de la superficie de las estrellas. Asimismo el perfil de Voigt está representado por una integral que sólo es posible calcular por métodos numéricos, de manera que la precisión con la que se puede extraer información de una línea experimental, cuando se le ajusta con ese perfil, se ha venido incrementando conforme evolucionan los métodos numéricos de integración.

\section{3.- LA FUNCION DE DISPERSION DEL PLASMA (FDP)}

La función de dispersión del plasma (FDP) apareció en la física entre los años cincuentas y sesentas como resultado de analizar los fenómenos de interacción de ondas electromagnéticas con cúmulos de partículas cargadas en movimiento asi como la propagación de ondas electrostáticas de pequeña amplitud en plasmas maxwellianos, es decir, plasmas en los que las velocidades de los electrones obedecen 
a la distribución de Maxwell [4]. Sin embargo, la FDP es proporcional a la función de error compleja, la cual es ampliamente conocida [5]. Se trata de un ejemplo más en el que el estudio de un fenómeno nuevo, como lo era en su tiempo la propagación de ondas en plasmas, conduce al uso de una función conocida como es la función de error compleja, para la descripción del fenómeno. Es sabido que al resolver el problema de propagación de ondas de pequeña amplitud en plasmas maxwellianos se obtiene la FDP, la cual contiene dos tipos generales de información, a saber: el primero relacionado con el espectro de frecuencias de la onda que se propaga, y el segundo relacionado con la distribución de velocidades de los electrones en el plasma. Es decir, la FDP contiene información acerca de la onda que se propaga y acerca del medio en el que ocurre la propagación. Los detalles serán analizados en el capítulo II donde se mostrará que esta información está implícitamente contenida en la variable compleja de la cual es función la FDP. Esta información se manifiesta claramente en las partes real e imaginaria de la FDP. La parte imaginaria es proporcional a una integral de convolución en la que intervienen un espectro de frecuencias lorentziano, característico de una onda amortiguada centrado en la frecuencia $\omega_{0}$ y una distribución gaussiana que caracteriza a la distribución de velocidades de los electrones. A esta integral de convolución se le conoce desde hace mucho tiempo en espectroscopía y es precisamente el perfil de Voigt, el cual ha sido usado para simular y ajustar líneas espectrales. Con la parte real de la FDP se pueden describir espectros de dispersión. Este tipo de líneas se presenta por ejemplo en las espectroscopias de RPE y de RMN y contienen la misma información física que las líneas de absorción. En el presente trabajo, éste es el fundamento para usar la FDP en la descripción de las formas de las lineas espectrales y para proponer nuevos modelos de perfiles que sirvan para ajustar lineas espectrales experimentales más complicadas como por ejemplo líneas asimétricas. Existe, sin embargo, un importante aspecto adicional de la FDP que la 
hace idónea, desde un punto de vista formal, para la descripción no sólo de líneas de absorción sino también de dispersión. Este aspecto es que las partes real e imaginaria de la FDP son las transformadas de Hilbert una de la otra; dicho de otra manera, ambas partes están conectadas por las relaciones de Kramers-Krönig y por lo tanto satisfacen ciertas propiedades de simetría. Lo importante de este asunto es que las interacciones radiación-materia (por ejemplo la propagación de ondas en plasmas) responsables de la aparición de líneas de absorción y dispersión, al obedecer al principio de causalidad, se describen vía relaciones de dispersión. La FDP es una de estas relaciones y por lo tanto su empleo en espectroscopia garantiza solidez y formalidad en la descripción de formas de línea. Sin negar la utilidad de los modelos empíricos de formas de línea que abundan en la literatura, los modelos basados en una relación de dispersión como lo es la FDP, son más generales y como se mencionó arriba incluyen automáticamente los perfiles de absorción así como los de dispersión que son también útiles en espectroscopía. Como se verá en el capítulo $V$ del presente trabajo, los perfiles de dispersión y absorción sirven también para construir gráficas y datos DISPA (DISPersión vs Absorción) de líneas espectrales, de los que se puede extraer información espectral de interés. 


\section{CAPITULO II}

\section{EL PERFIL DE VOIGT: SU RELACION CON LA FUNCION DE ERROR COMPLEJA Y CON LA FUNCION DE DISPERSION DEL PLASMA}

\section{1.- INTRODUCCION}

En este capítulo se describirá la relación que existe entre el perfil de Voigt, la función de error compleja y la función de dispersión del plasma.

El perfil de Voigt, que describe con buena aproximación la forma de líneas espectrales de absorción o emisión afectadas por mecanismos perturbadores externos de naturaleza aleatoria, es conocido desde hace mucho tiempo. Inicialmente fué usado en astrofísica para determinar la temperatura de la atmósfera de las estrellas. La agitación caótica de los átomos emisores en el gas caliente que constituye la atmósfera estelar hace que las líneas espectrales se ensanchen. Mediante la determinación de la magnitud de este ensanchamiento adicional, que se obtiene de la deconvolución del perfil de Voigt, se estima la temperatura que prevalece en una región dada de la atmósfera de la estrella.

Por otra parte, es sabido que el perfil de Voigt está emparentado con la función de error [9]; ésto se mostrará en forma explícita más adelante. Esta función surge del análisis estadístico de fenómenos aleatorios con el empleo de gaussianas y sirve para determinar la probabilidad de un evento en un intervalo de valores de la variable de estas distribuciones. La función de error es muy conocida y está ampliamente tabulada en sus versiones real y compleja [5]. 
A su vez, la FDP es de origen más reciente; aparece en las épocas en que comienza a manifestarse gran interés por comprender la física de los plasmas, campo multidisciplinario de la Física que está sirviendo como herramienta en el estudio $y$ posible control de la energia de fusión nuclear [4]. Desde el punto de vista matemático, la FDP está ligada estrechamente con la función de error compleja. Debido a su origen, la función de dispersión del plasma contiene información lorentziana y gaussiana que la relaciona con la función de error y por lo tanto con el perfil de Voigt. En forma más general, la función de dispersión del plasma se puede relacionar con los procesos de absorción y dispersión de energía oscilatoria en distintos medios. Esos procesos son de interés en espectroscopía y en otras áreas de la Física y de la Ingenieria, pues proporcionan información acerca de los medios en que ellos ocurren. De acuerdo con estas ideas, resulta de interés encontrar explicitamente la relación que existe entre las funciones de error, de dispersión del plasma y el perfil de Voigt. El objetivo de este capítulo es obtener dicha relación. La justificación para hacer ésto es que el perfil de Voigt y su transformada de Hilbert, pueden ser evaluados a través de la función de error, cuyas propiedades son bien conocidas, 0 a través de la FDP de la cual se tienen disponibles muchas formas aproximadas. Una ventaja adicional es que las expresiones para la absorción y para la dispersión en varias espectroscopias, pueden ser generalizadas usando la FDP de tal forma que queden considerados los efectos ensanchadores de tipo aleatorio. Aún más, en un capítulo posterior se introducirá un parámetro de asimetria en las expresiones para absorción y dispersión. De esta forma, el alcance de este trabajo se incrementa considerablemente ya que será posible analizar no solamente líneas simétricas sino tambien perfiles de línea asimétricos que se presentan frecuentemante en espectroscopía.

La manera de proceder en este capitulo, consistirá primero en relacionar el perfil de Voigt y su transformada de Hilbert con la función de error compleja. Posteriormente se relacionará dicho perfil con la función de dispersión del plasma para finalmente 
proponer un nuevo método para estimar la temperatura y la densidad electrónicas de un plasma, extrayendo por deconvolución las componentes lorentziana y gaussiana de dicha función.

\section{2.- EL PERFIL DE VOIGT COMO LA CONVOLUCION DE LA FUNCION LORENTZIANA CON LA DISTRIBUCION GAUSSIANA}

El concepto de convolución (ver capitulo I) es general y se presenta en varias ramas de la ciencia y de la ingeniería y describe la acción de un instrumento observador o detector sobre un observable físico. Esta acción consiste en que durante la observación, nunca se mide el verdadero valor del observable sino el resultado de tomar un promedio pesado de éste con una función perturbadora característica del instrumento observador. Dicho de otra manera, no se miden los valores verdaderos del observable sino la convolución formada por la distribución de valores del observable con la función de peso que es característica del sistema detector. Esta función es comúnmente conocida como la "resolución del sistema detector" y frecuentemente tiene la forma de una gaussiana, significando ésto que el efecto perturbador del sistema detector sobre el observable es de origen aleatorio.

Como consecuencia de lo anterior, todas las observaciones físicas están limitadas por la resolución del instrumental detector y por ésto, el fenómeno de convolución está siempre presente en la operación de medición independientemente de que dentro del sistema mismo, se convuelvan otros perfiles que le sean característicos. Matemáticamente, el proceso anterior se describe mediante una integral que involucra a una señal de inteligencia (o cualquier información de interés proveniente del sistema bajo estudio) y a la perturbación externa. Esta integral recibe varias denominaciones, entre ellas integral de convolución, de superposición, de composición, o bien integral de Duhamel [10], etc. Por ejemplo, si la señal de interés es una radiación resonante 
de anchura $\Gamma$, las imperfecciones del sistema de detección actúan sobre ella de manera tal que producen un ensanchamiento adicional en el espectro de frecuencias de la señal detectada. La presencia del fenómeno de convolución es general y abarca todas las ramas de la Física y de la Ingeniería en las que las señales están sometidas durante su detección y análisis a procesos perturbadores de orígen aleatorio.

Las líneas espectrales, correspondientes a las señales emitidas o absorbidas por un sistema aislado que puede ser descrito por medio de un modelo basado en una carga eléctrica que se mueve como un oscilador armónico amortiguado, tienen un perfil de Lorentz de anchura natural $\Gamma_{L}$ en la mitad del máximo [11]. En efecto, la transformada de Fourier de una función armónica amortiguada es una función lorentziana. Por ejemplo, si concebimos al sistema como un conjunto de átomos en los que los electrones están armónicamente ligados al núcleo (aproximación dipolar [11]) y si dicho sistema está sujeto a una intensa agitación térmica (átomos emisores en un gas calientel, entonces el efecto neto de los corrimientos Doppler producidos en la radiación emitida por los átomos puede sobrepasar con mucho la anchura "natural" $\Gamma_{L}$. En tal caso el perfil de la linea adopta una forma gaussiana con anchura $\Gamma_{G}$ en la mitad del máximo. Los perfiles intermedios, obtenidos por medio de la convolución de formas de línea lorentzianas y gaussianas, se denominan perfiles de Voigt [3].

En otros casos, como por ejemplo en la espectroscopia de retrodispersión de Rutherford (RBS) en la que se detectan protones retrodispersados por una muestra, bajo ciertas condiciones los espectros obtenidos pueden ser representados mediante la convolución de una función rectangular, que representa al substrato, con una distribución gaussiana que incluye a la dispersión del haz, a la resolución instrumental y al "straggling" (dispersión de alcances) [12]. En tal caso el perfil resultante no es voigtiano porque no es la convolución de una función lorentziana con una distribución gaussiana. 
Además del gran interés práctico que tiene la omnipresencia de la convolución como concepto físico, el teorema asociado con ella [13] ofrece un punto de partida para algunas aplicaciones relacionadas con la deconvolución tanto de perfiles voigtianos como de no voigtianos $[12,14]$.

La convolución $h(x)$ de dos funciones $f(u)$ y $g(u)$ [13] se escribe en forma general como:

$$
h(x)=\int_{-\infty}^{\infty} f(u) g(x-u) d u \equiv f(x)^{*} g(x) . \quad(I I-1)
$$

De la expresión (II-1) se observa que la función $g(u)$ se incorpora al integrando después de haber efectuado sobre ella las siguientes operaciones que se ilustran en la Figura II-1. Primero, $g(u)$ se translada hacia la derecha por una cantidad $x$ a lo largo del eje $u$ y después se refleja con respecto al nuevo eje vertical. El orden de estas operaciones no importa ya que el producto final es el mismo. En efecto, la función $g(u)$ puede primero ser reflejada alrededor de su eje vertical y después transladada hacia la derecha por una cantidad $x$. Esto significa que si se representan $f(u)$ y $g(u)$ en un mismo diagrama, estas funciones se verían como se muestra en la Figura II-1.

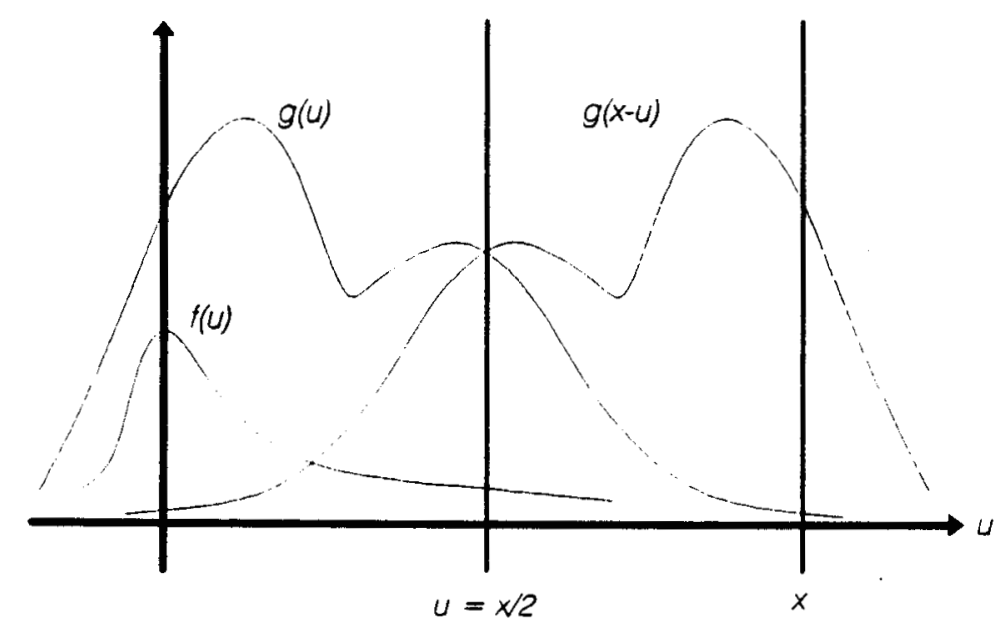

Figura ll-1.-Funciones $f(u)$ y $g(x-u)$ que participan en la integral de convolución. 
En la Figura $11-1$ se aprecia también que el resultado neto de las operaciones indicadas sobre $g(u)$ es "doblarla" o reflejarla sobre si misma alrededor del punto $u=x / 2$. Debido a ésto a la operación de convolución se le denomina "faltung", del alemán, que significa doblar. En esta figura la forma de la función de peso, $g(u)$, se ha elegido asimétrica para hacer notar el efecto del doblado sobre ella. Es claro que tal efecto no se observa si la función es simétrica, es decir si $g(u-x)=g(x-u)$.

Un aspecto práctico de (II-1) es que, la expresión para $h(x)$ puede ser considerada como un promedio pesado de $f(u)$ en el que la función $g(x-u)$ desempeña el papel de función de peso. En estas condiciones, la función $h(x)$ es una función "suavizada", considerando a $g(x-u)$ como función "suavizadora". Otra caracteristica importante de la convolución expresada en (II-1) es la propiedad de conmutatividad, la cual se demuestra como sigue.

El cambio de variable $u=v+x$, en (II-1), produce la expresión

$$
h(x)=\int_{-\infty}^{\infty} f(v+x) g(-v) d v
$$

y el cambio adicional $v=-u$, conduce a

$$
h(x)=\int_{-\infty}^{\infty} g(u) f(x-u) d u \equiv g(x)^{*} f(x) \cdot \quad(I I-1 a)
$$

De (II-1) y (II-1a) se concluye que

$$
h(x)=f(x) * g(x)=g(x) * f(x), \quad(I I-2)
$$

lo cual es consecuencia de las operaciones combinadas de corrimiento y reflexión consideradas con anterioridad. Debido a esta propiedad, la expresión (II-1a) representa también el hecho de que $h(x)$ es el promedio pesado de $g(u)$ en el que $f(x-u)$ desempeña ahora el papel de función de peso. 
Cuando se realiza la convolución de un perfil lorentziano $L(v)$ con una distribución gaussiana $G(v)$ la cual está escrita en términos de una variable $v$ y un parámetro a adimensionales que serán definidos más adelante, se obtiene el llamado perfil de Voigt [3, 9]. La convolución puede hacerse de cualquiera de las dos maneras indicadas en (II-2). Por comodidad en el manejo algebraico, se elegirá la lorentziana como la función a doblar. Dado que el presente trabajo estará basado en perfiles voigtianos, a continuación se obtendrá la expresión para tales perfiles.

Supóngase un sistema físico caracterizado por una señal representada por una función lorentziana $L(v)$, sometido a la acción de una perturbación aleatoria representada por una gaussiana $C(\vee)$; donde $\vee$ puede tener unidades de frecuencia, de energía, de número de canal, etc., dependiendo del tipo de espectroscopía de que se trate. La intensidad de la señal espectral será proporcional a la convolución, $I(v)$. de $L(v)$ y $G(v)$, esto es,

$$
I(v)=\int_{-\infty}^{\infty} L\left(v-v^{\prime}\right) G\left(v^{\prime}\right) d v^{\prime}
$$

Esta expresión puede ser considerada como la suma infinita y continua de una distribución gaussiana de lorentzianas de la misma anchura, cada una centrada en $v_{i}$. La lorentziana centrada en $v_{1}$, tiene una altura dada por $L\left(v_{1}\right)$.

Sean $L(v)$ y $G(v)$ las expresiones normalizadas al área (el area bajo la curva tiene una magnitud igual a la unidadl para la lorentziana y la gaussiana, respectivamente:

$$
\begin{gathered}
L\left(v-v^{\prime}\right)=\frac{\Gamma_{L}}{2 \pi}\left[\frac{1}{\left(v-v_{0}-v^{\prime}\right)^{2}+\frac{\Gamma_{l}^{2}}{+}}\right], \\
G(v)=\frac{1}{\sqrt{2 \pi \sigma^{2}}} e^{-\frac{v^{2}}{2 \sigma^{2}}},
\end{gathered}
$$


donde $v_{0}$ es la frecuencia central de la señal de inteligencia, o la desviación estándar de la gaussiana y $\Gamma_{L}$ es el ancho lorentziano a la mitad del máximo.

Efectuando la convolución de estas dos expresiones, la intensidad $I(v)$ de la linea espectral observada es proporcional a la integral

$$
I(v)=\int_{-\infty}^{\infty} \frac{\Gamma L}{2 \pi} \frac{1}{\left[\left(v-v_{0}-v^{\prime}\right)^{2}+\frac{r_{l}^{2}}{4}\right]} \frac{e^{-\frac{v^{2}}{2 a^{2}}}}{\sqrt{2 \pi \sigma^{2}}} d v^{\prime} \cdot(I I-3)
$$

Después de hacer algunas manipulaciones algebraicas y definiendo, de acuerdo con Posener [9], la variable adimensional de frecuencia como

$$
v=\frac{2\left(v-v_{0}\right) \sqrt{\ln 2}}{\Gamma_{G}}, \quad(I I-4 a)
$$

y el parámetro adimensional

$$
a=\frac{\Gamma_{L}}{\Gamma_{G}} \sqrt{\ln 2},
$$

la expresión (II-3) puede ser reescrita como

$$
I(v, a)=\frac{1}{\sqrt{2 \pi \sigma^{2}}} H(v, a),
$$

donde la expresión

$$
H(v, \alpha)=\frac{a}{\pi} \int_{-\infty}^{\infty} \frac{e^{-y^{2}} d y}{(v-y)^{2}+\alpha^{2}}, \quad(1 /-6)
$$

es conocida como el perfil de Voigt. 
Nótese de (II-4b) que a mide las contribuciones relativas lorentziana y gaussiana al perfil de Voigt. En las definiciones (II-4a) y (II-4b), $\Gamma_{G}$ es la anchura gaussiana total en la mitad del máximo, en las unidades determinadas por el tipo de espectroscopía de que se trate y está relacionada con la desviación estandar, $\sigma$, por la fórmula

$$
\Gamma_{G}=2 \sqrt{2 \ln 2} \sigma .
$$

La expresión $l(v, a)$ en (II-5), está normalizada, pues proviene de la convolución de dos funciones normalizadas, es decir

$$
\int_{-\infty}^{\infty} I(u, a) d v=1 .
$$

Una forma de línea espectral obtenida experimentalmente en varias espectroscopías puede ser descrita mediante el perfil de Voigt, siempre y cuando las perturbaciones sobre el perfil lorentziano de emisión o absorción puedan ser representadas por una distribución gaussiana.

\section{3.- RELACION DEL PERFIL DE VOIGT CON LA FUNCION DE ERROR COMPLEJA}

Considérese cualquier sistema oscilatorio que pueda simularse mecánicamente por una masa $m$ suspendida de un resorte con constante de fuerza $k$, sujeto a una resistencia friccional $f$ y excitado por una fuerza oscilatoria de frecuencia angular $\omega$. Es sabido que, en el estado estacionario, la masa oscila con la misma frecuencia que la fuerza motriz, pero no siempre con la misma fase. El movimiento de la masa queda completamente caracterizado al determinar las componentes del desplazamiento, en el dominio de la frecuencia, que están en fase y $90^{\circ}$ fuera de fase con respecto a la fuerza motriz. En particular ocurre que cuando la frecuencia de la fuerza motriz es igual a la frecuencia propia $\omega_{o}$ del oscilador, no existe componente 
fuera de fase del desplazamiento. Esto significa que la absorción de energía que realiza el oscilador a expensas de la fuente que aplica la fuerza, se lleva a cabo con la máxima eficiencia. Las magnitudes de los desplazamientos en fase $x$ y fuera de fase $x$ para un amplio intervalo de frecuencias de oscilación que incluya la frecuencia propia $\omega$ 。 tienen los espectros mostrados en la Figura II-2. La expresión algebraica de estos desplazamientos en el dominio de la frecuencia pueden ser obtenidos de la expresión (III-19).
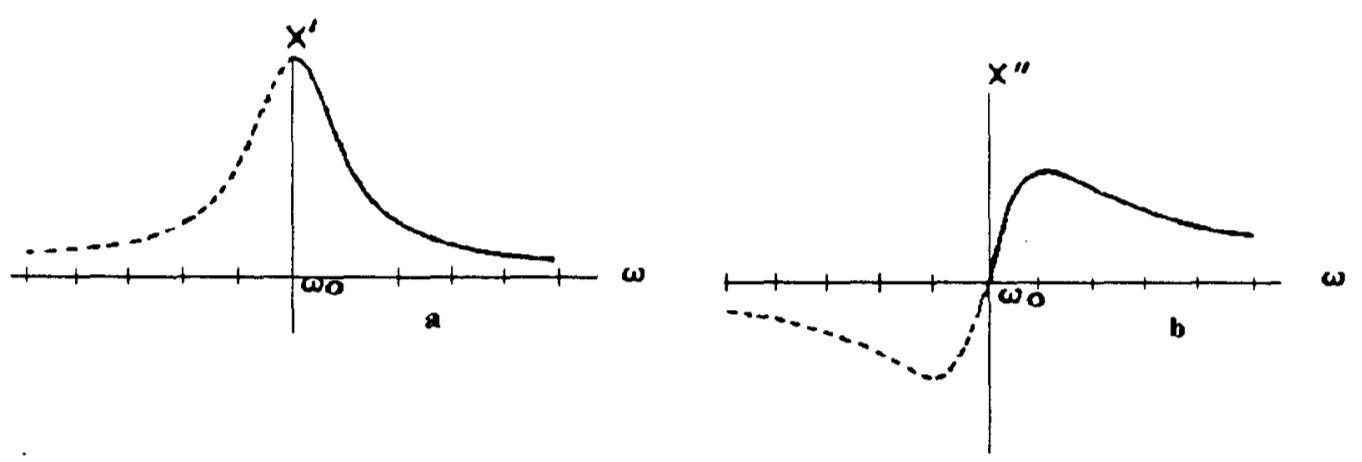

Figurall-2.-Amplitud de desplazamiento del oscilador forzado con fricción. a) Respuesta en fase. b) Respuesta fuera de fase.

En el contexto de la óptica la respuesta fuera de fase representa la variación en frecuencia del índice de refracción del medio considerado, en tanto que la respuesta en fase representa la absorción de energía por parte del medio. A la respuesta fuera de fase se le denomina espectro de dispersión porque la variación del indice de refracción conduce a que la luz blanca se disperse en sus componentes.

Una descripción análoga es válida en otras espectrocopías como RPE, RMN, etc. en las que también se produce el par dispersión-absorción descritas por las relaciones de dispersión del sistema y que están conectadas por medio de la transformada de 
Hilbert [15]. Como se demostrará al final de esta sección, las expresiones que se obtendrán para absorción y dispersión están contenidas en la función de error de argumento complejo.

Manipulando la expresión (II-6) para el perfil de Voigt, es posible escribirla en términos de la función de error compleja $w(z)$. Para lograrlo la exponencial que aparece en el integrando de (II-6) será representada como la transformada de Fourier

$$
e^{-y^{2}}=\frac{1}{\sqrt{\pi}} \int_{0}^{\infty} e^{-\frac{x^{2}}{4}}(\cos y x) d x .
$$

Al substituir esta expresión en (II-6) e introducir el cambio de variable $t=v-y$, se llega a que

$$
H(v, \alpha)=\frac{a}{\pi} \frac{1}{\sqrt{\pi}} \int_{0}^{\infty} e^{-\frac{x^{2}}{4}}(\cos v x) \int_{-\infty}^{\infty}\left(\cos \frac{t x}{t^{2}+a^{2}}\right) d t d x .
$$

Esta expresión se reduce a la siguiente, que es par en la variable $u$ [16]

$$
H(u, a)=\frac{1}{\sqrt{\pi}} \int_{0}^{\infty} e^{-\frac{x^{2}}{4}-x a}(\cos v x) d x \quad, \quad(I I-8)
$$

de la cual se obtiene (Ref. [16], p. 480)

$$
H(v, c)=\operatorname{Re}\left[e^{-z^{2}} \operatorname{erfc}(-i z)\right], \quad(I I-9)
$$

ó, en términos de la función de error con argumento complejo $w(z)$ [5]:

$$
H(u, a)=\operatorname{Re}[w(:)], \quad(I I-10)
$$

donde la variable compleja $z$ está representada por

$$
z=v+i a
$$


Por sus utilidad en aplicaciones posteriores, conviene resaltar la importancia de la variable (II-11). En virtud de que : está escrita en términos de la variable adimensional real u de la Ec. (II-4a) y del parámetro adimensional a de la Ec. (II-4b), ella contiene toda la información acerca de las componentes lorentziana y gaussiana en el perfil de Voigt. Esta información puede ser recuperada al deconvolucionar dicho perfil de manera que por ejemplo, para aplicaciones en otros campos se pueden obtener los parámetros de interés que describen el amortiguamiento de una oscilación y la magnitud de los mecanismos que la perturban. Al final de este capítulo se presentará un ejemplo en el que se propone una nueva manera para determinar temperatura $v$ densidad electrónicas en un plasma.

Por otra parte, es interesante observar que empleando las definiciones (II-4a,b, 7), la variable $\approx$ se puede manipular para obtener la expresión:

$$
\frac{1}{\because z z^{*}}=\frac{2 \sigma^{2}}{\left(v-v_{0}\right)^{2}+\left(\frac{r_{L}}{2}\right)^{2}},
$$

la cual es proporcional al perfil lorentziano, y también se puede probar que

$$
e^{-\cdots * *}=e^{-u^{2}} e^{-\frac{\left(v-v_{0}\right)^{2}}{2 \sigma^{2}}}
$$

es proporcional a la distribución gaussiana.

Atendiendo a la expresión (II-8), ésta indica que el perfil de Voigt $H(u, a)$, puede escribirse como la transformada de Fourier de la función

$$
e^{-\frac{x^{2}}{4}-x a}
$$

lo cual es correcto puesto que de acuerdo con el teorema de convolución [13], la expresión (II-8) debe ser la transformada inversa de Fourier del producto de las transformadas de la gaussiana ensanchadora y de un perfil lorentziano. En efecto, 
$\exp \left(-x^{2} / 4\right)$ es la transformada inversa de Fourier de la gaussiana ensanchadora $y$ $\exp (-|x| a)$ es la transformada inversa de Fourier de una lorentziana; ambas funciones descritas en términos de variables adimensionales de frecuencia.

Obsérvese que si en la expresión (II-8), a $\rightarrow 0$, la función $H(v, a)$ tiende a la gaussiana lo cual es consistente con el hecho de que la transformada de Fourier de una gaussiana es otra gaussiana. Análogamente, para valores suficientemente grandes de $a$, el lado derecho de (II-8) tiende a una lorentziana como es de esperarse.

La expresión (II-10) muestra la relación que existe entre la función compleja de errory el perfil de absorción $H(U, a)$ con efectos ensanchadores incluidos. Para tener una descripción completa del problema bajo estudio, es necesario investigar con qué se identifica la parte imaginaria de la función de error compleja. Para lograr tal fin se hace uso del hecho que en ausencia de fenómenos no lineales, es decir cuando la amplitud de la respuesta de un sistema es proporcional a la amplitud de la excitación, el perfil de absorción está relacionado con el de dispersión por medio de la transformada de Hilbert [17]. Por lo tanto, se aplicará en ambos miembros de (II-8) esta transformada con respecto a la variable $v$ [13]. En vista de que la transformada de Hilbert de $\operatorname{Cos} v x$ es - Sen $v x$ [18], el perfil de dispersión $D(v, a)$ que se obtiene es

$$
D(u, a)=-\frac{1}{\sqrt{\pi}} \int_{0}^{\infty} e^{-\frac{x^{2}}{4}-x a}(\operatorname{sen} u x) d x
$$

que es una función impar en la variable $v$ [18]. Adicionalmente es fácil demostrar que esta última relación puede escribirse en términos de la parte imaginaria de la función de error compleja, de modo que:

$$
D(u, c)=-\operatorname{Im}[w(\because)]
$$

Si se desea que $w(\because)$ represente a una relación de dispersión que tome en consideración fenómenos aleatorios que producen ensanchamiento de las lineas 
espectrales del sistema bajo consideración, es necesario que $H(v, a)$ y $D(v, a)$ satisfagan las relaciones de Kramers-Krönig para una función compleja $f\left(x_{0}\right)=u\left(x_{0}\right)+i v\left(x_{0}\right)$, donde $[13,17]$ :

$$
\begin{aligned}
& u\left(x_{0}\right)=\frac{1}{\pi} P \int_{-\infty}^{\infty} \frac{v(x)}{x-x_{0}} d x, \\
& u\left(x_{0}\right)=-\frac{1}{\pi} P \int_{-\infty}^{\infty} \frac{u(x)}{x-x_{0}} d x,
\end{aligned}
$$

donde la letra "P" escrita ańtes de las integrales indica que se debe tomar la parte principal de ellas. Entonces combinando adecuadamente las expresiones (II-10) y (II-13), se obtiene

$$
H(v, a)-i D(v, a)=w(\ddot{z})
$$

Es decir, la función $w(z)$ se puede expresar como una combinación compleja de $H(v, a)$ y $D(v, a)$, que son funciones reales.

Ahora bien, en vista de que $H(v, c)$ y $D(v, a)$ han sido combinadas para satisfacer las relaciones de Kramers-Krönig, entonces $w(z)$ representa una relación de dispersión que toma en cuenta fenómenos aleatorios que producen ensanchamiento de las lineas espectrales emitidas por el sistema bajo consideración.

La ecuación (11-14) es relevante y útil ya que una combinación de las expresiones que describen matemáticamente la forma en que se absorbe y se dispersa la energia en un sistema físico, reproduce a la función de error compleja. Nótese que los efectos ensanchadores como agitación térmica, etc. quedan incluidos en esta discusión. 
Resulta conveniente además, relacionar $H(u, a)$ con la función de dispersión del plasma[19], no sólo por la comodidad de manejar funciones conocidas como ella, que se pueden calcular con el grado de precisión deseado [20] sino también por la disponibilidad de formas aproximadas y por la posibilidad de generalizar el uso de expresiones como $H(v, a)$ y $D(v, a)$ en espectroscopía.

En vista de que es conocida la relación entre la función de error compleja $w(z)$ con la función de dispersión del plasma $Z(\zeta)$. [9] se podria escribir de inmediato la relación que hay entre $H($ l a l $)$ con dicha función de dispersión. Sin embargo, será útil glosar la forma en que surge la función $Z(\zeta)$ y describir la manera en que los parámetros que caracterizan un plasma no magnetizado se incluyen en la variable compleja de la cual depende esa función de dispersión. Procediendo en esta forma se obtendrá fácilmente la relación entre $H(v, a)$ y $Z(\zeta)$ y además se verá con claridad la idea que se propondrá al final de este capítulo sobre una aplicación para diagnóstico de plasmas [6]. En la siguiente sección se procederá a deducir la función de dispersión del plasma $Z(\zeta)$ donde la variable $\zeta$ puede escribirse en términos de la variable 2 y del parámetro a contenidos en (II-11).

\section{4.- RELACION DEL PERFIL DE VOIGT CON LA FUNCION DE DISPERSION DEL PLASMA [21]}

La función de dispersión del plasma aparece cuando se resuelve el problema de la propagación de ondas electrostáticas o longitudinales $(\vec{K} \times \vec{E}=0)$ de pequeña amplitud en un plasma en el que la función de distribución de las velocidades de las partículas que lo componen es maxwelliana. Se hace una suposición inicial que consiste en considerar al gas de iones del plasma como un fondo inmóvil de carga positiva, cuya finalidad es mantener la cuasineutralidad del plasma, en tal forma que la masa 
$M$ de los iones sea tan grande comparada con la masa $m$ de los electrones que su movimiento prácticamente no afecte la electrodinámica del problema más allá de una pequeña corrección de orden $m / \mathrm{M}$.

Este es un problema típico y ya clásico en Física de Plasmas en el que están acoplados los movimientos de las partículas cargadas con la propagación de ondas electrostáticas en el plasma. Los movimientos de las partículas, en este caso los electrones del plasma, se describen a través de la evolución de su función de distribución $f(\overrightarrow{\mathbf{r}}, \overrightarrow{\mathbf{v}}, t)$ en el espacio fase. El comportamiento de $f(\overrightarrow{\mathbf{r}}, \overrightarrow{\mathbf{v}}, \imath)$, a su vez, está descrito por la ecuación de Vlasov [22]. Por otra parte, la propagación de las ondas en el medio considerado se describe por medio de las ecuaciones de Maxwell en las que las cargas y sus flujos se representan como los momentos de orden cero y uno de la función de distribución, respectivamente. El conjunto de ecuaciones que describe al sistema es

$$
\begin{aligned}
& \frac{\partial f(\overrightarrow{\mathbf{r}}, \overrightarrow{\mathbf{v}}, t)}{\partial t}+\overrightarrow{\mathbf{v}} \cdot \nabla_{r} f(\overrightarrow{\mathbf{r}}, \overrightarrow{\mathbf{v}}, \iota)+\frac{e}{m}\left(\overrightarrow{\mathbf{E}}+\frac{\overrightarrow{\mathbf{v}} \times \overrightarrow{\mathbf{H}}}{c}\right) \cdot \nabla_{v} f(\overrightarrow{\mathbf{r}}, \overrightarrow{\mathbf{v}}, t)=0,(I I-15) \\
& \nabla \cdot \overrightarrow{\mathrm{E}}=4 \pi e\left[\int f(\overrightarrow{\mathbf{r}}, \overrightarrow{\mathrm{v}}, t) d \overrightarrow{\mathrm{v}}-n_{\imath}\right], \quad(I /-16) \\
& \nabla \times \overrightarrow{\mathbf{E}}=-\frac{1}{c} \frac{\partial \overrightarrow{\mathrm{H}}}{\partial t} \\
& \nabla \cdot \vec{H}=0 \\
& (I I-17) \\
& (11-18) \\
& \nabla \times \overrightarrow{\mathbf{H}}=\frac{1}{c} \frac{\partial \overrightarrow{\mathbf{E}}}{\partial t}+\frac{4 \pi e}{c} \int f(\overrightarrow{\mathbf{r}}, \overrightarrow{\mathbf{v}}, t) \overrightarrow{\mathbf{v}} d \overrightarrow{\mathbf{v}} \\
& (11-19)
\end{aligned}
$$

En la ecuación (II-15) $\nabla_{r}$ y $\nabla_{u}$ son los operadores vectoriales nabla en los espacios físico $y$ de velocidades, respectivamente. En estas ecuaciones $n_{\text {, es la }}$ densidad fija de iones. 
El primer término en (II-15) describe la variación temporal implícita de $f(\overrightarrow{\mathbf{r}}, \overrightarrow{\mathbf{v}}, t)$, el segundo es un término de arrastre que describe el cambio temporal de $f(\overrightarrow{\mathbf{r}}, \overrightarrow{\mathbf{v}}, t)$ debido a que los elementos de volúmen del plasma han sido arrastrados con una velocidad $\vec{v}$ y el tercer término representa el cambio temporal en $f(\overrightarrow{\mathbf{r}}, \overrightarrow{\mathbf{v}}, t)$ bajo la acción de la fuerza electromagnética aplicada a los elementos de volúmen del plasma. La función $f(\overrightarrow{\mathbf{r}}, \overrightarrow{\mathbf{v}}, t)$ está normalizada al número $N$ de electrones en el plasma:

$$
\int f(\overrightarrow{\mathbf{r}}, \overrightarrow{\mathbf{v}}, t) d \overrightarrow{\mathbf{r}} d \overrightarrow{\mathbf{v}}=N . \quad(I /-20)
$$

El sistema de ecuaciones (II-15)-(II-19) tiene 9 de ellas y 9 incógnitas que corresponden a las componentes de $\vec{V}, \vec{E}, y \vec{H}$.

Para el problema particular de propagación de ondas longitudinales de pequeña amplitud se supone, además, que la función de distribución $f(\overrightarrow{\mathbf{r}}, \overrightarrow{\mathbf{v}}, t)$ difiere muy poco de la distribución de orden cero, $f_{0}(\vec{v})$, que prevalece en el plasma en ausencia de toda perturbación. Esta distribución es espacial y temporalmente uniforme y es tal que

$$
\int f_{0}(\vec{v}) d \vec{v}=n_{e}
$$

donde $n_{e}$ es la densidad de electrones que a su vez es igual a $n_{l}$, la densidad iónica de fondo. Se propone además que a orden cero no existen campos eléctricos ni magnéticos en el plasma. Estos sólo surgen en el plasma a primer orden debido a la perturbación causada por la onda. Lo anterior implica que no hay campos $\vec{E} \circ \vec{H}$ aplicados externamente $y$ que

$$
\int \vec{v} f_{0}(\vec{v}) d \vec{v}=0
$$


lo cual significa que, a orden cero, no hay corrientes en el plasma y por las suposiciones anteriores se sigue que, a este orden, las ecuaciones (II-15)-(II-19) son identicamente cero.

De acuerdo con las suposiciones anteriores, la función de distribución del plasma se escribirá como la función de distribución de orden cero que sólo depende de la velocidad, más una corrección que depende también de las coordenadas y del tiempo para tomar en cuenta el efecto de la onda sobre el movimiento de los electrones, es decir

$$
f(\overrightarrow{\mathbf{r}}, \overrightarrow{\mathbf{v}}, t)=f_{0}(\overrightarrow{\mathbf{v}})+f_{1}(\overrightarrow{\mathbf{r}}, \overrightarrow{\mathbf{v}}, t),
$$

lo cual significa que el problema se analizará solamente hasta el primer orden de aproximación y por lo tanto se dice que el sistema de ecuaciones será "linealizado". Esta linealización se realiza con base en la suposición, ya hecha, de que la amplitud de la onda electrostática es suficientemente pequeña de tal forma que se evita la aparición de efectos no lineales que no se considerarán aquí. Así pues, el sistema linealizado de ecuaciones se escribe de la siguiente manera:

$$
\begin{array}{cc}
\frac{\partial f_{1}(\overrightarrow{\mathbf{r}}, \overrightarrow{\mathbf{v}}, t)}{\partial t}+\overrightarrow{\mathbf{v}} \cdot \nabla_{r} f_{1}(\overrightarrow{\mathbf{r}}, \overrightarrow{\mathbf{v}}, t)+\frac{e}{m}\left(\overrightarrow{\mathrm{E}}_{1}+\frac{\overrightarrow{\mathbf{v}} \times \overrightarrow{\mathbf{H}}_{1}}{c}\right) \cdot \nabla_{u} f_{0}(\overrightarrow{\mathbf{v}})=0,(I I-2+) \\
\nabla \cdot \overrightarrow{\mathrm{E}}_{1}=4 \pi e \int f_{1}(\overrightarrow{\mathbf{r}}, \overrightarrow{\mathbf{v}}, t) d \overrightarrow{\mathbf{v}}, & (I I-25) \\
\nabla \times \overrightarrow{\mathbf{E}}_{1}=-\frac{1}{c} \frac{\partial \overrightarrow{\mathbf{H}}_{1}}{\partial t}, & (I I-26) \\
\nabla \cdot \overrightarrow{\mathbf{H}}_{1}=0, & (I I-27) \\
\nabla \times \overrightarrow{\mathbf{H}}_{1}=\frac{1}{c} \frac{\partial \overrightarrow{\mathbf{E}}_{1}}{\partial t}+\frac{4 \pi e}{c} \int \overrightarrow{\mathbf{v}} f_{1}(\overrightarrow{\mathbf{r}}, \overrightarrow{\mathbf{v}}, t) d \overrightarrow{\mathbf{v} .} & (I I-28)
\end{array}
$$


En lo que sigue se considerarán solamente ondas electrostáticas o longitudinales. Esta condición implica que la onda no tiene componente magnética y por lo tanto $\overrightarrow{\mathbf{H}}_{1}=0$. La condición también implica que el vector de propagación $\overrightarrow{\mathbf{k}}$ es paralelo al vector de campo eléctrico $\vec{E}_{1}$. Suponiendo que la variación espacial de la onda se puede representar como $\exp (i \overrightarrow{\mathbf{K}} \cdot \overrightarrow{\mathbf{r}})$ entonces la condición de paralelismo entre $\overrightarrow{\mathbf{K}}$ y $\vec{E}_{1}$ se transcribe como $\nabla \times \vec{E}_{1}=0$. lo cual significa que

$$
\overrightarrow{\mathrm{E}}_{1}=-\nabla \phi_{1} \text {. }
$$

Es necesario hacer notar que la descomposición en ondas puramente longitudinales y puramente transversales puede hacerse sólo si $f_{0}(\vec{v})$ es isotrópica; de otra manera se presentan otros modos de propagación como ocurre en un plasma magnetizado en el que $f_{0}(\vec{v})$ no es isotrópica. Bajo estas condiciones, el sistema (II-24)-(II-28) se reduce a

$$
\begin{array}{cc}
\frac{\partial f_{1}(\overrightarrow{\mathbf{r}}, \overrightarrow{\mathbf{v}}, t)}{\partial t}+\overrightarrow{\mathbf{v}} \cdot \nabla_{r} f_{1}(\overrightarrow{\mathbf{r}}, \overrightarrow{\mathbf{v}}, t)-\frac{e}{m}\left(\nabla \phi_{1}\right) \cdot \nabla_{u} f_{0}(\overrightarrow{\mathbf{v}})=0, & (I I-30) \\
\nabla^{2} \phi_{1}=-4 \pi e \int f_{1}(\overrightarrow{\mathbf{r}}, \vec{v}, t) d \overrightarrow{\mathbf{v}} & (I I-31)
\end{array}
$$

El sistema de ecuaciones (II-30) y (II-31) fué resuelto por Vlasov [22] para el caso de longitudes de onda grandes y en forma general por Landau [23], como un problema de valor inicial. La solución de este sistema de ecuaciones se presenta en el Apéndice. En este Apéndice se muestra cómo del sistema (11-30, 11-31) se obtiene la relación de dispersión del presente problema. Esta relación de dispersión es

$$
1=\frac{4 \pi e^{2}}{m k^{2}} \int_{c}^{\frac{d F_{0}(u)}{d u}} \frac{\left(A-\frac{i s}{k}\right.}{u-11)}
$$


donde $c$ es el contorno de integración en el plano complejo $u$ y las variables $k, s$ están definidas en el Apéndice.

Considérese ahora el caso particular en el que $F_{0}(u)$ es la distribución de Maxwell (altas temperaturas y bajas densidades):

$$
F_{0}(u)=\frac{n_{e}}{\sqrt{\pi} u_{T}} \exp \left(-\frac{u^{2}}{v_{T}^{2}}\right)
$$

En esta expresión $v_{T}$, la velocidad térmica, se define como:

$$
v_{T}=\sqrt{\frac{2 k_{B} T_{e}}{m}}
$$

donde $k_{B}$ es la constante de Boltzmann y $T_{\text {e }}$ es la temperatura del gas de electrones.

Empleando (II-32) la ecuación (A-11) adquiere la forma

$$
1=\frac{-1}{\sqrt{\pi} k^{2} \lambda_{D}^{2}} \int_{c} \frac{t e^{-t^{2}}}{t-\zeta} d t
$$

donde

$$
\begin{aligned}
& t=\frac{u}{v_{T}}, \\
& \zeta=\frac{i s}{k v_{T}},
\end{aligned}
$$

Y

$$
\lambda_{D}^{2}=\frac{k T}{4 \pi n_{e} e^{2}} \quad, \quad k_{D}=\frac{2 \pi}{\lambda_{D}} \quad(I I-37)
$$

es el cuadrado de la longitud de Debye [24]. La cantidad

$$
\omega_{p}=\sqrt{\frac{4 \pi n_{e} e^{2}}{m}}, \quad(I I-38)
$$


es conocida como la frecuencia de plasma [24] la cual será usada más adelante. En (II-36) s es la frecuencia compleja que se usa en la transformada de Laplace.

La ecuación (II-34) describe el comportamiento (absorción y dispersión) de ondas electrostáticas de pequeña amplitud en un plasma maxwelliano. Despues de un poco de álgebra, esta ecuación se puede escribir como:

$$
1+k^{2} \lambda_{D}^{2}+\zeta Z(\zeta)=0
$$

donde

$$
Z(\zeta)=\frac{1}{\sqrt{\pi}} \int_{c} \frac{e^{-t^{2}}}{t-\zeta} d t
$$

es la función de dispersión del plasma [4].

Para generar las tablas de $Z(\zeta)$, se usa la expresión:

$$
Z(\zeta)=\frac{1}{\sqrt{\pi}} \int_{-\infty}^{\infty} \frac{e^{-y^{2}}}{y-\zeta} d y
$$

y la ecuación diferencial para $Z(\zeta)$ [4].

Estamos ahora en posición de obtener la relación entre el perfil de Voigt y la FDP a partir de la racionalización de (II-41). En efecto, denotando a $\zeta$ como $\zeta=v+i a$, la racionalización de $(11-41)$ es

$$
Z(\zeta)=\frac{1}{\sqrt{\pi}} \int_{-\infty}^{\infty} \frac{(y-v) e^{-y^{2}} d y}{(y-v)^{2}+a^{2}}+i \frac{a}{\sqrt{\pi}} \int_{-\infty}^{\infty} \frac{e^{-y^{2}} d y}{(y-v)^{2}+a^{2}}, \quad \quad(I I-42)
$$

de la cual, con ayuda de (II-6), se obtiene inmediatamente que

$$
H(u, a)=\frac{1}{\sqrt{\pi}} \operatorname{lm}[Z(\zeta)]
$$

Lógicamente, el perfil de dispersión se obtiene como 


$$
D(v, a)=\frac{1}{\sqrt{\pi}} \operatorname{Re}[Z(\zeta)] \quad, \quad(I I-43 b)
$$

En esta forma los perfiles $H(u, a)$ y $D(u, a)$ son expresados en términos de la FDP que es una función conocida y tabulada, de la cual existen muchas formas aproximadas en la literatura algunas de las cuales serán analizadas en el capitulo III.

Para usos posteriores, es conveniente observar que en la expresión (II-42) las partes real e imaginaria de $Z(\zeta)$ están conectadas por medio de la transformada de Hilbert con respecto a la variable $u$. como corresponde a una relación de dispersión $[13,17,18]$. También vale la pena resaltar las propiedades de simetría de (II-42) que posteriormente serán también de utilidad, esto es

$$
\begin{array}{rlr}
\operatorname{Re} Z(v, a) & =-\operatorname{Re} Z(-v, a), & (I I-44 a) \\
\operatorname{Im} Z(v, a) & =\operatorname{Im} Z(-v, a), & (I I-44 b) \\
Z\left(\zeta^{*}\right) & =-[Z(-\zeta)]^{*} . & (I I-44 c)
\end{array}
$$

\section{5.- UN METODO PARA ESTIMAR TEMPERATURA Y DENSIDAD ELECTRONICAS EN UN PLASMA}

En esta sección se propondrá un método para estimar la temperatura $T_{\text {e }}$ y la densidad $n_{e}$ electrónicas de un plasma, basado en la deconvolución de la parte simétrica (absorción) de la función de dispersión del plasma [II-43a]. El presente método tiene su base en la observación de que la función $Z(\zeta)$ contiene de origen, toda la información acerca de la propagación con amortiguamiento de una onda electrostática en un plasma maxwelliano con temperatura y densidad electrónicas $T_{e}$ y $n_{e}$, 
respectivamente. Es conveniente hacer notar aqui que la expresión (II-43) indica que $\operatorname{Im}[Z(\zeta)]$ es la convolución de una gaussiana y una lorentziana, escrita en términos de variables adimensionales.

Como se ha indicado anteriormente, la gaussiana representa a la distribución maxwelliana de velocidades de los electrones del plasma y la lorentziana representa a la forma de línea o espectro de frecuencias de una onda electrostática sondeadora linealizada, de frecuencia angular $\omega_{0}$ y número de onda $k$, que se propaga con amortiguamiento en el plasma.

Para extraer la información contenida en la función de dispersión $Z(\zeta)$ en el presente problema, es necesario analizar primero el contenido de la variable $\zeta$ definida en (II-36) en la que $s$ es la variable compleja de frecuencia que aparece en en la transformada de Laplace y que, por conveniencia, se escribirá como:

$$
s=\gamma-i \omega .
$$

Substituyendo (II-45) en (II-36), se llega a que

$$
\zeta=\frac{\left(\omega-\omega_{0}\right)+i \gamma}{k v_{T}},
$$

donde se ha introducido una frecuencia fija $\omega_{0}$ correspondiente a una onda electrostática de sondeo que se hace propagar en el plasma y y es el amortiguamiento que sufre la onda al interactuar con los electrones del plasma. Por otra parte $v_{T}$, definida en (II-33), puede escribirse como

$$
v_{T}=\sqrt{\frac{2 k_{B} T_{e}}{m}}=\sqrt{2} \sigma_{v}, \quad(11-47)
$$

por lo que $\sigma_{v}=\sqrt{k_{B} T_{e} / m}$ es la desviación estandar, en unidades de velocidad, de la gaussiana correspondiente a la distribución de velocidades (cf ec. II-32). Analogamente a la expresión (II-7), que tiene unidades de frecuencia 


$$
\Gamma_{G}=2 \sqrt{2 \ln 2} \sigma,
$$

se puede construir una relación equivalente pero con unidades de velocidad

$$
\Gamma_{G}^{\prime}=2 \sqrt{2 \ln 2} \sigma_{v} .
$$

Substituyendo (II-48) en (II-47), se tiene:

$$
v_{T}=\frac{\Gamma_{\dot{G}}}{2 \sqrt{\ln 2}} .
$$

Substituyendo ahora (II-49) en (II-46), se llega a

$$
\zeta=\frac{2\left(\omega-\omega_{0}\right) \sqrt{\ln 2}}{k \Gamma_{G}}+i \frac{2 \gamma \sqrt{\ln 2}}{k \Gamma_{G}},
$$

donde $\Gamma_{G}$ tiene unidades de velocidad y $k \Gamma_{G}$ unidades de frecuencia angular. Por otra parte, recordando que la transformada de Fourier de la función de intensidad de radiación emitida por un oscilador que tiene una constante de amortiguamiento $y$ es un perfil lorentziano de semianchura $Y$, se puede decir que la anchura del espectro de frecuencias que se detecta para la onda electrostática que se propagó en el plasma es $2 \gamma=\Gamma_{L}$. Con estas consideraciones la última expresión se escribe finalmente como

$$
\zeta=\frac{2\left(\omega-\omega_{0}\right) \sqrt{\ln 2}}{\Gamma_{G}}+i \frac{\Gamma_{L} \sqrt{\ln 2}}{\Gamma_{G}},
$$

donde

$$
\Gamma_{G}=k \Gamma_{G}^{\prime} .
$$

Comparando (II-50) con (II-11) se concluye que, de acuerdo con las expresiones (II-4), la variable $\zeta$ es idéntica a $z=v+i a$ definida en (II-11) y por lo tanto

$$
\zeta=u+i a l \equiv: \therefore
$$

por lo que, en lo sucesivo, en lugar de $\zeta$ se escribirá $z$. 
La deconvolución del perfil de absorción que se detecta de la onda electrostática sondeadora, produce los valores $\Gamma_{L}$ y $\Gamma_{G}$ que caracterizan los perfiles lorentziano y gaussiano que intervienen en la convolución. La deconvolución puede efectuarse numéricamente usando métodos reportados con anterioridad $[25,26]$.

Para completar el análisis en esta propuesta de aplicación y finalmente obtener los valores de $T_{e}$ Y $n_{e}$, sólo resta proporcionar expresiones para $\Gamma_{L}$ y $\Gamma_{G}$ en términos de esos parámetros. Una expresión para $\Gamma_{G}$ en términos de $T_{e}$, se obtiene combinando (II-51), (II-48) y (II-47) para una onda sondeadora con número de onda $k$. Esta expresión es:

$$
\Gamma_{G}=2 k \sqrt{\frac{2(\ln 2) k_{B} T_{e}}{m}}
$$

Con el objeto de proporcionar una expresión para $\Gamma_{L}$ en términos de $T_{e}$ y $n_{e}$, se supondrá que la amplitud de la onda decae en tal forma que $\Gamma_{L}$ es el amortiguamiento de Landau $[23,24,27]$

$\Gamma_{L}=2 y=2 \omega_{D} \sqrt{(\pi / 8)}\left(k_{D} / k\right)^{3} \exp \left(-3 / 2-k_{D}^{2} / 2 k^{2}\right), \quad k \ll k_{D},(I I-53)$ donde $k_{D}$ y $\omega_{p}$ están definidas en (II-37) y (II-38), respectivamente. Como se ve de su definición, $\omega_{p}$ depende de la densidad electrónica no perturbada $n_{e}$ y $k_{D}$ depende de $T_{e}$ y $n_{e}$. La expresión (II-53) es válida sólo cuando el número de onda de la señal de sondeo satisface la condición $k \ll k_{0}$.

Finalmente, efectuando la deconvolución numérica de la señal detectada cuya distribución de frecuencias esta centrada en $\omega_{\text {。 }} y$ que se propagó en el plasma, se obtienen los valores de $\Gamma_{L} y \Gamma_{G}$. De éstos, se pueden obtener los valores de $n_{e} Y$ $T_{\text {e }}$ por medio de las relaciones (II-52) y (II-53). Esta sugerencia para determinar los parámetros $n_{e}$ y $T_{e}$ en un plasma se publicó en la referencia [6]. 
En este punto es adecuado proponer un experimento orientado a probar el presente método sugerido para determinar la temperatura y la densidad electrónicas en un plasma. El experimento puede ser realizado en una "máquina $Q$ " que se caracteriza por generar plasmas quietos y de duración arbitraria. Podria también intentarse realizar el experimento en una descarga de resplandor. El plasma producido en esta descarga es más fácil de obtener ya que sólo se necesita una fuente de energía de $C D$, una cámara con un par de electrodos y el gas de trabajo para obtener el plasma. Sin embargo en este tipo de plasma el grado de ionización es muy bajo y seria necesario una alta sensitividad del sistema detector para observar algún amortiguamiento de la onda sondeadora. 


\section{CAPITULO III}

\section{FORMAS APROXIMADAS PARA LOS PERFILES DE ABSORCION Y DISPERSION DE LINEAS ESPECTRALES}

\section{1.-INTRODUCCION}

Como se mencionó en el capítulo anterior, la función de dispersión del plasma $Z(z)$ juega un papel central en las ecuaciones de dispersión de ondas en un plasma. Sin embargo, en este trabajo se pretende ampliar el campo de aplicaciones de la función $Z(z)$ para incluir la espectroscopía donde el perfil de Voigt es usado frecuentemente para estudiar la estructura de lineas espectrales $[6,19,25]$.

Las expresiones para el perfil de Voigt $H(u, a)(11-6,11-43 a)$ y para la función de dispersión del plasma $Z(z)$ (II-42), son exactas y deben evaluarse como tales para ajustarse a cualquier espectro experimental que contenga contribuciones de tipos gaussiano y lorentziano. En algunas ocasiones, sin embargo, se sabe a priori que un espectro contiene una contribución más dominante de un tipo que del otro lel espectro puede tener un mayor contenido gaussiano que lorentziano o viceversa) y entonces, para ahorrar tiempo, puede hacerse uso de expresiones aproximadas.

Las expresiones algebraicas aproximadas para los perfiles de Voigt $y$ sus correspondientes de dispersión, son de utilidad en espectroscopía para simplificar cálculos analíticos o realizar rápidamente estimaciones numéricas. De acuerdo con lo presentado hasta ahora sobre la relación de estos perfiles con la función de dispersión del plasma $Z(z)$, se deduce que las expresiones aproximadas de ésta última pueden también ser útiles en espectroscopia. Sin embargo es necesario tener cuidado y 
asegurarse de que las expresiones aproximadas que se propongan cumplan con las propiedades de simetría de $Z(z)$ (II-44), asi como con las relaciones de Kramers-Krönig $[13,15,17,28,29]$, a fin de que dichas expresiones puedan ser consideradas como relaciones de dispersión a pesar de ser sólo aproximaciones. En efecto, las propiedades de absorción y de dispersión de un sistema dependen de la frecuencia (o de la energía) de la radiación con la que éste interactúa. Esas dos propiedades están conectadas a través de las relaciones de dispersión o relaciones de Kramers-Krönig. Con estas condiciones satisfechas, las relaciones aproximadas se pueden aplicar formal y confiablemente a problemas de espectroscopía para ajustar espectros de absorción y dispersión experimentales o para simularlos.

En este capítulo se analizarán tres ejemplos tomados de la literatura, relacionados con sendas formas aproximadas para la función de dispersión del plasma $Z(\because)$. El perfil de Voigt para la absorción se puede deducir de ellas de acuerdo con la ecuación (II-43a) o la expresión (7) de la Ref. [19].

En este capítulo se comprobará que las formas aproximadas para la función de dispersión del plasma reportadas por Fried et al.[30], por P. Martin et al. [31] y por Robinson [32], satisfacen las propiedades de simetria de $Z(\approx)$. En las dos primeras referencias las formas aproximadas para $Z(z)$ cumplen las relaciones de Kramers-Krönig por lo que pueden ser consideradas como relaciones de dispersión. Sin embargo, en el trabajo de Robinson [32] estas relaciones se cumplen solamente para uno de los casos presentados alli y resulta que este caso es precisamente aquél para el que la forma aproximada se ajusta muy mal al perfil exacto de $Z(z)$. En los tres trabajos citados, los autores ponen énfasis en el provecho que se obtiene al 
acelerar cálculos numéricos y al presentar expresiones analíticas sencillas, pero ellos no constatan explícitamente si sus aproximaciones satisfacen las relaciones de Kramers-Krönig.

Como cuarto ejemplo de formas aproximadas para los perfiles de absorción y dispersión, en este capitulo se propondrá además una nueva para los perfiles de Voigt y de dispersión basada en una expresión aproximada del perfil de Voigt $[26,33]$. La pareja de perfiles resultante consta de expresiones analíticas sencillas pero que satisfacen los requisitos ya mencionados, de tal suerte que este par de perfiles componen una relación de dispersión. El par es útil para aquellos casos en los que la contribución gaussiana es pequeña, es decir $a \gg \frac{1}{\sqrt{2}}$; (véase la definición de a en $(11-4 b))$.

Antes de analizar los ejemplos mencionados, es conveniente introducir algunos conceptos que serán de utilidad al estudiar con más detenimiento las partes de absorción y dispersión de un espectro y en particular para manejar los cuatro ejemplos citados de formas aproximadas para los perfiles de absorción y dispersión. Dichos conceptos son los de causalidad y su relación con el comportamiento analítico de las relaciones de dispersión en el plano complejo de la frecuencia. Como complemento se mostrará que las partes real e imaginaria de una relación de dispersión en términos de la parte real de la frecuencia compleja, están relacionadas por la transformada de Hilbert ó, lo que es lo mismo, por las relaciones de Kramers-Krönig. El orígen de estas relaciones se encuentra en la transformada de Fourier de funciones causales del tiempo.

\section{2.-FUNCIONES CAUSALES}

Todas las formas de onda físicas que son funciones del tiempo son causales, es decir, están definidas a partir de un instante dado del tiempo, por ejemplo, a partir de 
$t=0 \quad y$ son presentadas como respuesta, consecuencia o efecto de una causa establecida con anterioridad. Este aspecto causal desemboca en el hecho de que la transformada de Fourier de estas funciones se compone de dos partes que son funciones de la frecuencia, a saber: una parte real, simétrica con respecto a una "frecuencia de resonancia" $\omega_{0}$, que representa un espectro de absorción y una parte imaginaria, antisimétrica con respecto a $\omega_{0}$, que representa un espectro de dispersión. Estas dos partes están relacionadas por medio de la transformada de Hilbert y se denominan relaciones de Kramers-Krönig. Gráficamente ésto se ilustra en la Figura III-1.

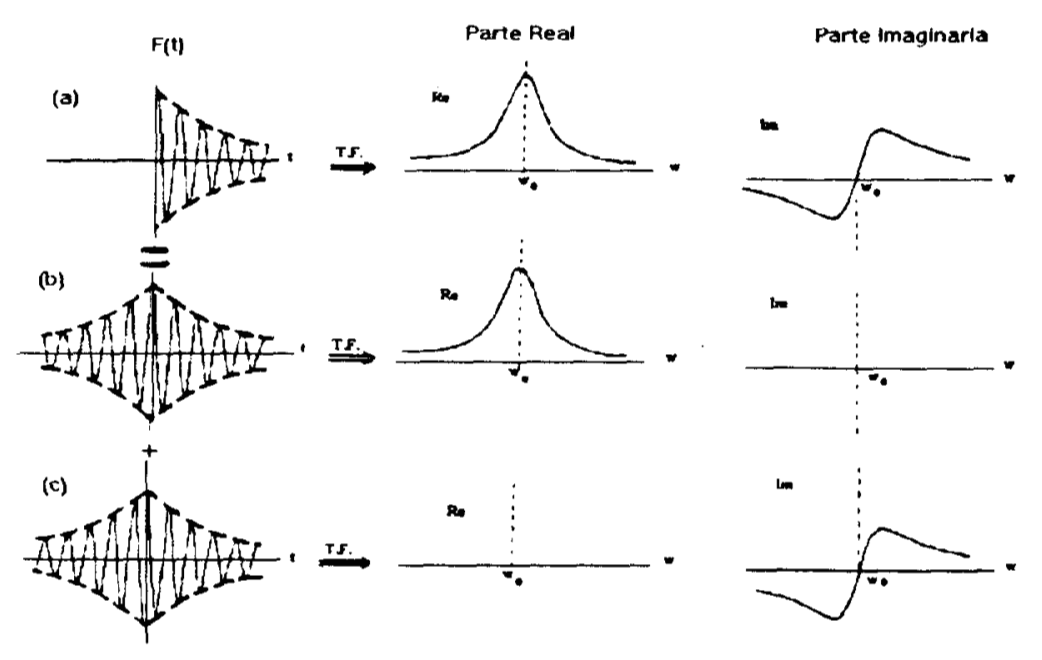

Figura III-1.- La transformada de Fourier de la función causal $F(t)$ que comienza en $t=0$ y que decrece exponencialmente, consta de una parte real, simétrica y en este caso lorentziana mas una parte imaginaria antisimétrica. En el texto se describe cada parte de esta figura.

En la parte (a) de la Fig. III-1 se muestra un ejemplo de una función causal hipotética, armónica y amortiguada exponencialmente que puede ser descompuesta en una función par del tiempo y en una función impar del tiempo trazadas en el lado izquierdo del segundo $y$ tercer renglones de esta figura, respectivamente. La 
transformada de Fourier de la función causal $F(t)$ que comienza en $t=0$ y que decrece al transcurrir el tiempo, consta de una parte real simétrica más una parte imaginaria antisimétrica. En la parte $(b)$ de la Fig. III-1, la función $F(t)$ referida en la parte (a) de la Fig. III-1, se complementa para $t<0$ con su reflejo simétrico con respecto a $t=0$ dando origen a una función tipo coseno decreciente en ambos sentidos del tiempo. La transformada de Fourier de esta nueva función, que es par, es puramente real y es simétrica con respecto a $\omega_{0}$. En la parte $(c)$ de la Fig. III-1 la función $F(t)$ de la parte (a) de la Fig. III-1, se complementa para $t<0$, con su reflejo antisimétrico con respecto a $t=0$ dando origen a una función tipo seno decreciente en ambos sentidos del tiempo. La transformada de Fourier de esta nueva función, que es impar, es puramente imaginaria y es antisimétrica con respecto a $w_{0}$. Obsérvese que la función $F(t)$ en Fig. III-1a, se puede obtener como la suma de las funciones del tiempo de las partes $(b)$ y $(c)$ de la Fig. III-1 [34].

En la siguientes dos secciones se describirán las relaciones que ligan a las partes real e imaginaria de la transformada de Fourier de una función causal, asi como la relación que existe entre los conceptos de analiticidad y causalidad.

\section{3.-RELACIONES DE DISPERSION Y RELACIONES DE KRAMERS-KRÖNIG}

El concepto de relaciones de dispersión entró en la Física con los trabajos de Krönig y de Kramers en óptica, al estudiar la dispersión de la luz en distintos medios $[28,29]$. A partir de entonces se da el nombre de "relaciones de dispersión" a cualquier par de ecuaciones, en la variable real $\omega$, en el que una de ellas relaciona a la parte real de una función compleja con la transformada de Hilbert de su parte imaginaria, y la otra relaciona a la parte imaginaria de dicha función con la transformada de Hilbert de su parte real. Estas relaciones son el análogo integral de las condiciones de 
Cauchy-Riemann para la existencia de la derivada de una función compleja, en un punto y su vecindad y por to tanto para su analiticidad o regularidad en esa región $[13,34]$. En lo que sigue se presentará la obtención de las relaciones de dispersión a la manera de Arfken [13] y Wyld [35]; véase también [36].

Considérese una función $f(z)$ de la variable compleja $z$, que es holomórfica o analítica en el semiplano superior (SPS) y sobre el eje real de manera que $f^{\prime}(z)$ existe en toda esa región. Esta función puede ser escrita en términos de sus partes real e imaginaria como:

$$
f(x, y)=u(x, y)+i v(x, y) .
$$

Considérese además la condición:

$$
\lim _{z \rightarrow \infty \rightarrow \infty}|f(z)|=0, \quad 0 \leq \operatorname{Arg} z \leq \pi,
$$

que se demanda con el objeto de que la integral de $f(z)$ a lo largo del contorno que rodea a un semicírculo infinito en SPS se nulifique. Por lo tanto, de acuerdo con el teorema integral de Cauchy, se puede escribir

$$
f\left(z_{0}\right)=\frac{1}{2 \pi i} \oint \frac{f(z) d z}{z-z_{0}}
$$

donde el contorno cerrado está compuesto por la semicircunferencia infinita en SPS y el eje real y donde $z_{\text {。 }}$ es un punto en SPS dentro del contorno cerrado de integración, véase la Fig. III-2. En virtud de que la integral sobre la semicircunferencia infinita se nulifica, (III-2) se convierte en la siguiente integral sobre el eje real:

$$
f\left(z_{0}\right)=\frac{1}{2 \pi i} \int_{-\infty}^{\infty} \frac{f(x) d x}{x-z_{0}}
$$




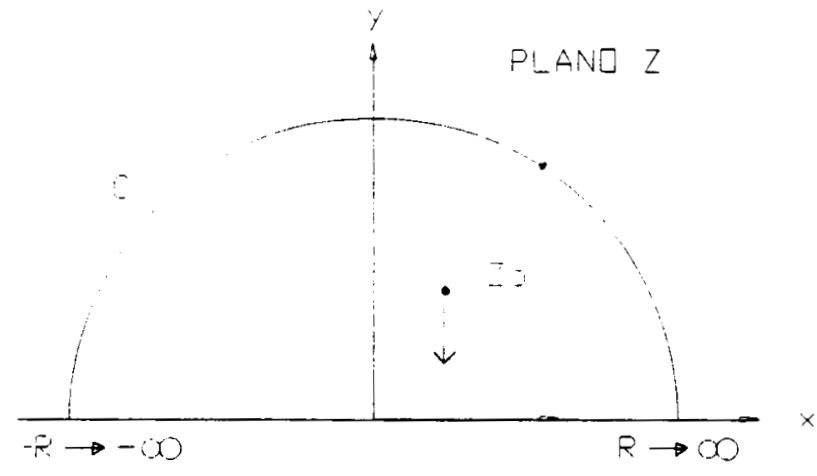

Figura III-2.- Contorno de integración para la integral en (III-2).

Con el objeto de expresar (III-3) en términos de la variable real $x$, se permite que el punto $z_{0}$ se acerque, desde arriba, hacia el eje real $(y \rightarrow 0)$ y se coloque en $x_{0}$; ver Fig. III-3.

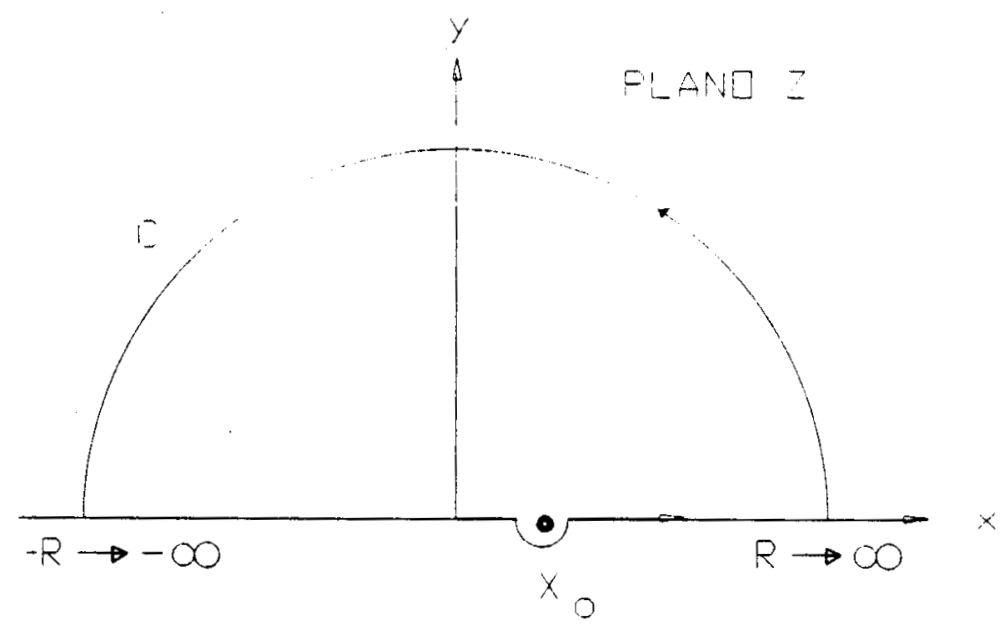

Figura III-3.- El polo $z_{0}$ de la Fig. III-2 ha sido transladado y colocado sobre el eje real. 
En estas condiciones, la expresión (III-3) se escribe como:

$$
f\left(x_{0}\right)=\frac{1}{2 \pi i}\left[P \int_{-\infty}^{\infty} \frac{f(x) d x}{x-x_{0}}+i \pi f\left(x_{0}\right)\right] . \quad(1 / 1-4)
$$

donde $P$ indica que se debe tomar la parte principal de la integral, es decir toda la integral excepto la contribución en el polo $x$ 。 la cual está considerada en el segundo término dentro del paréntesis de (III-4). Simplificando la expresión (III-4) se obtiene

$$
f\left(x_{0}\right)=\frac{1}{\pi i} P \int_{-\infty}^{\infty} \frac{f(x) d x}{x-x_{0}} .
$$

Esribiendo $f\left(x_{0}\right)$ en términos de sus partes real e imaginaria se obtiene

$$
f\left(x_{0}\right)=u\left(x_{0}\right)+i v\left(x_{0}\right)=\frac{1}{\pi} P \int_{-\infty}^{\infty} \frac{u(x) d x}{x-x_{0}}-i \frac{1}{\pi} P \int_{-\infty}^{\infty} \frac{u(x) d x}{x-x_{0}},
$$

de donde se sigue que

$$
\begin{array}{ll}
u\left(x_{0}\right)=\frac{1}{\pi} P \int_{-\infty}^{\infty} \frac{u(x) d x}{x-x_{0}} . & (111-7 a) \\
u\left(x_{0}\right)=-\frac{1}{\pi} P \int_{-\infty}^{\infty} \frac{u(x) d x}{x-x_{0}} . & (111-7 b)
\end{array}
$$

Las expresiones (III-7) son las relaciones de dispersión o relaciones de Kramers-Krönig. De estas expresiones se observa que $u(x)$ es la transformada de Hilbert de $v(x)$ y ésta es la transformada de Hilbert, con signo negativo, de $u(x)$.

De (III-7a, b) se desprende que las paridades de $u(x)$ y $v(x)$ son tales que cuando una es par, la otra es impar. Por otra parte, $f(x)$ se obtiene a partir de la transformada de Fourier de una función física (real) $s(t)=s^{*}(t)$, es decir:

$$
f(x)=\int_{-\infty}^{\infty} e^{-\imath x t} s(t) d t
$$


Tomando el complejo conjugado en ambos miembros, se obtiene

$$
f^{*}(x)=\int_{-\infty}^{\infty} e^{i x t} s^{*}(t) d t
$$

donde el asterisco indica que se debe tomar el complejo conjugado de la función. Al cambiar el signo de $x$ en ambos miembros se obtiene

$$
f^{*}(-x)=\int_{-\infty}^{\infty} e^{-\imath x t} s^{*}(t) d t
$$

pero como $s(t)=s^{*}(t)$, se deduce entonces que

$$
f(-x)=f^{*}(x),
$$

de donde se obtiene que las partes real e imaginaria de $f(x)$ tienen la paridad

$$
\begin{array}{ll}
u(x)=u(-x), & (111-8) \\
v(x)=-v(-x), & (111-9)
\end{array}
$$

por lo que $u(x)$ y $u(x)$ puden ser usadas, respectivamente, para representar los fenómenos físicos de absorción y dispersión de energía radiante por parte de un sistema que interactúa con un campo de radiación.

Las paridades (III-8,9) son entonces consecuencia de que $f(x)$ sea la transformada de Fourier de una señal $s(t)$ física y por lo tanto real, en el dominio del tiempo.

Lo anterior puede sintetizarse diciendo que toda función de la variable compleja $z$ que sea analítica en SPS y sobre el eje real y que además cumpla con la condición

$$
\lim _{|z| \rightarrow \infty}|f(z)|=0, \quad 0 \leq \operatorname{Arg} z \leq \pi .
$$

es una relación de dispersión y sus partes real e imaginaria están relacionadas por la transformada de Hilbert, dando origen a las relaciones de Kramers-Krönig. 
El sentido físico de estas condiciones puramente matemáticas es el siguiente: De acuerdo con (1-6) y (11-1), la función de respuesta $\phi(t)$ de un sistema lineal a una función estímulo de entrada $g(t)$ puede ser escrita como la convolución:

$$
\phi(t)=\int K\left(t-t^{\prime}\right) g\left(t^{\prime}\right) d t^{\prime} . \quad(1 / 1-10)
$$

donde $K(t)$ es la función de Green del sistema [36].

El requisito impuesto por el principio de causalidad, es decir que la respuesta no anteceda al estímulo, significa que $K(t)=0$ para $t<0$. Por otra parte, las funciones $f(z)$ discutidas antes son las transformadas de Fourier de tales funciones de Green y la condición $K(t)=0$ para $t<0$ implica que la función

$$
f(\therefore)=\int e^{u: *} K(t) d t \text {. }
$$

no tiene singularidades en SPS y que $f(z) \rightarrow 0$ a medida que $|z| \rightarrow \infty$ en SPS [35, 131. Asi pues, el significado físico de estas relaciones es que el sistema bajo estudio obedece al principio de causalidad. Enseguida se ilustrará lo anterior mediante un ejemplo sencillo tomado de la Ref. [15], basado en la solución al problema del oscilador armónico.

\section{4.-EL OSCILADOR ARMONICO}

El movimiento en una dimensión del oscilador armónico amortiguado y forzado por una función $f(t)$, se describe por medio de la ecuación

$$
\ddot{x}+2 y x+w_{0}^{2} x=f(t)
$$

donde $\omega_{0}$ es la frecuencia natural del oscilador $y \quad y$ es la constante de amortiguamiento cuyo valor es 


$$
y>0 \quad \text {. }
$$

es decir, no se aceptan sistemas con "anti-amortiguamiento" que conducen a soluciones que crecen sin límite al transcurrir el tiempo. A continuación analizarán algunos aspectos de la oscilación.

\section{a).- Oscilaciones libres}

En el caso $f(l)=0$ (oscilación libre), la solución general de (III-12) es

$$
x(t)=\kappa_{1} e^{-l \omega_{1} t}+k_{2} e^{-i \omega_{2} t} . \quad(111-1 t)
$$

donde $k_{1}, k_{2}$ son constantes y $\omega_{1}, \omega_{2}$ son las raices de la ecuación

$$
\omega^{2}+2 i y \omega-\omega_{0}^{2}=0,
$$

es decir

$$
w_{1,2}= \pm\left(w_{0}^{2}-y^{2}\right)^{1 / 2}-i y
$$

Obsérvese que $\omega_{1,2}$ es un vector de magnitud constante $\omega_{0}$, en el plano complejo de la frecuencia.

Es muy importante para los fines de este ejemplo, observar también que los polos $w_{1,2}$ nunca están ni en SPS ni sobre el eje real (recuérdese que $\gamma>0$ ). Ver Figura III-4. 


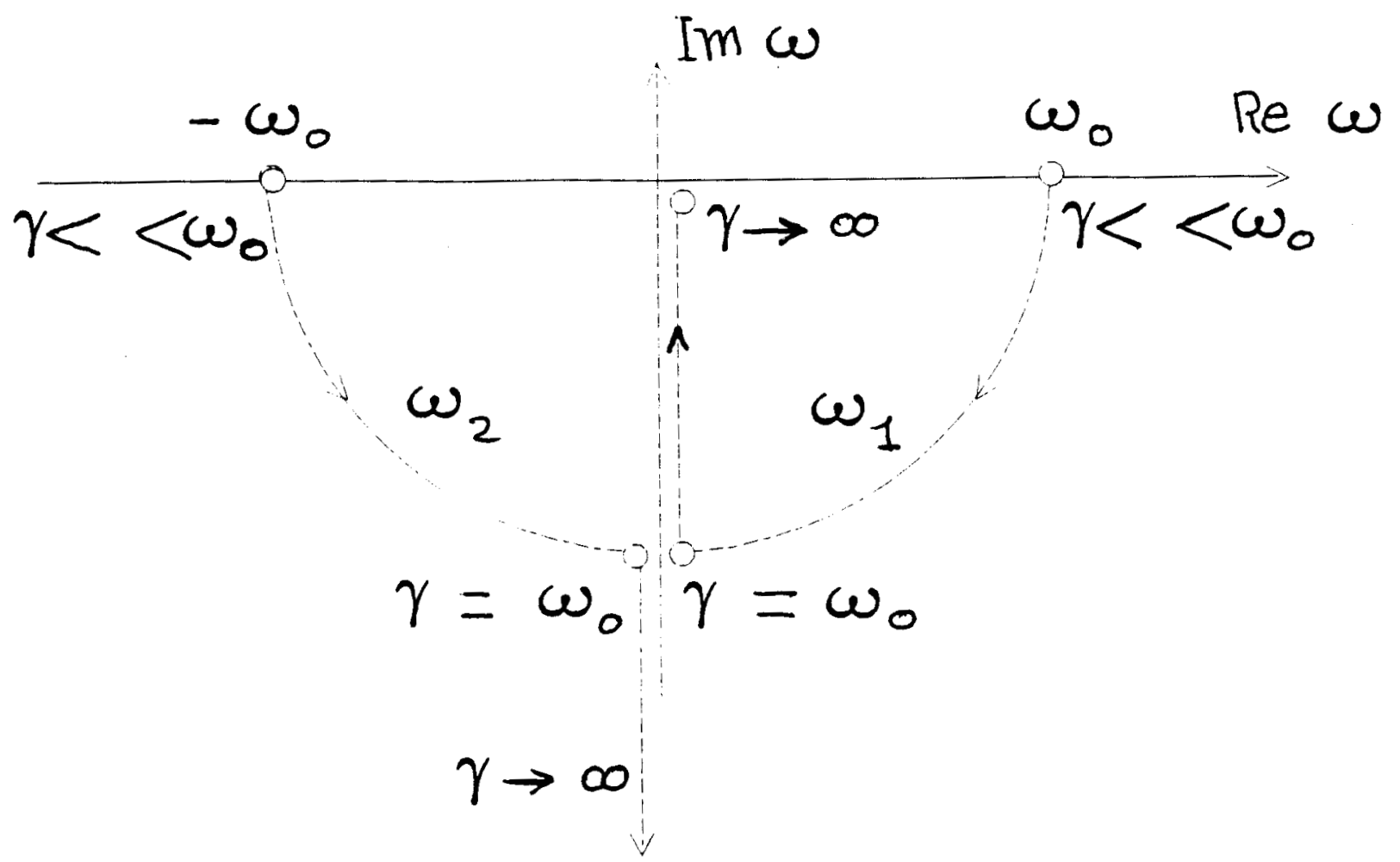

Figura lli-4. Comportamiento de los polos $\omega_{1}, \omega_{2}$ en el plano complejo de la frecuencia, en el intervalo $0<y<\infty$

La presencia de $y>0$ en la parte imaginaria de (III-16) produce en (III-14), oscilaciones que se amortiguan en un tiempo característico $1 / \gamma$. Nótese que si $Y$ toma valores negativos, se producen en (III-14) oscilaciones de amplitud exponencialmente creciente al transcurrir el tiempo. Estos casos no se considerán en el presente trabajo.

b).- Oscilaciones armónicamente forzadas

Si se aplica al oscilador la fuerza armónica descrita por la expresión 


$$
f(t)=F_{\omega} e^{-t \omega t}, \quad(111-17)
$$

se puede proponer la siguiente solución estacionaria

$$
x(t)=X_{\omega} e^{-t \omega t} .
$$

Substituyendo (III-17) y (III-18) en (III-12), se tiene que

$$
x_{\omega}=-\frac{F_{\omega}}{\omega^{2}+2 i \gamma \omega-\omega_{0}^{2}}=-\frac{F_{\omega}}{\left(\omega-\omega_{1}\right)\left(\omega-\omega_{2}\right)}, \quad(1 / 1-19)
$$

ó bien

$$
X_{\omega}=G(\omega) F_{\omega}
$$

con

$$
G(\omega)=-\frac{1}{\left(\omega-\omega_{1}\right)\left(\omega-\omega_{2}\right)}
$$

c).- La solución generalizada

La forma más general de fuerza que se puede aplicar al oscilador, se representa como la suma de todas las fuerzas posibles del tipo (III-17) mediante una integral de Fourier

$$
f(t)=\frac{1}{2 \pi} \int_{-\infty}^{\infty} F(\omega) e^{-i \omega t} d \omega
$$

donde

$$
F(\omega)=\int_{-\infty}^{\infty} f\left(t^{\prime}\right) e^{i \omega t} d t
$$

En este caso la solución de (III-12) se obtiene generalizando (III-18) por medio de una integral, de acuerdo con la naturaleza lineal del problema. La solución más general es pues 


$$
x(t)=\frac{1}{2 \pi} \int_{-\infty}^{\infty} X(\omega) e^{-i \omega t} d \omega . \quad(I I I-24)
$$

ó, empleando (III-20) con $F(\omega)$ en lugar de $F_{\omega}$ se obtiene

$$
x(t)=\frac{1}{2 \pi} \int_{-\infty}^{\infty} G(\omega) F(\omega) e^{-i \omega t} d \omega \quad \quad \quad(I / I-25)
$$

Substituyendo (III-23) en (III-25) e invirtiendo el orden de integración, suponiendo que las funciones se comportan bien para poder realizar este cambio, se tiene para la solución de (III-12) que

$$
x(t)=\int_{-\infty}^{\infty} g\left(t-t^{\prime}\right) f\left(t^{\prime}\right) d t \quad(111-26)
$$

con

$$
g(\tau)=\frac{1}{2 \pi} \int_{-\infty}^{\infty} G(\omega) e^{-i \omega \tau} d \omega, \quad(I l l-27)
$$

que es la transformada inversa de Fourier de $G(\omega)$. Nótese que (III-26) tiene la forma de una convolución como lo expresado en (III-10), siendo $f(t)$ la función de estímulo, $x(t)$ la función de respuesta y $g\left(t-t^{*}\right)$ la función de Green del oscilador.

En las expresiones (III-25, 26, 27) se pueden reconocer varios aspectos interesantes, a saber:

1.- La expresión (III-26) es una integral de convolución, como la presentada en (II-1) y (III-10), en la que intervienen la suma de fuerzas $f(t)$ y una función $g(t)$, cuya naturaleza se comprenderá enseguida.

2.- Si se elige $f\left(t^{*}\right)$ como la función delta de Dirac y se substituye $f\left(t^{\circ}\right)=\delta\left(t^{\circ}\right)$, en (III-26), la solución $x(t)$ de (III-12) es $g(t)$. Dicho de otra manera, $g(t)$ es la respuesta temporal del oscilador a una fuerza impulsiva instántanea. La función real $g(t)$ viene entonces a ser la 
función de Green del oscilador y $G(\omega)$ la transformada de Fourier de $g(t)$, que es la respuesta en frecuencia o espectro de frecuencias del oscilador a este estímulo impulsivo.

3.- Igualando (III-25) con (III-26) se recobra el teorema de convolución del análisis de Fourier, a saber:

$$
\frac{1}{2 \pi} \int_{-\infty}^{\infty} G(\omega) F(\omega) e^{-i \omega t} d \omega=\int_{-\infty}^{\infty} g\left(t-t^{\prime}\right) f\left(t^{\prime}\right) d t \quad, \quad(I I I-28)
$$

donde $G(\omega)$ y $F(\omega)$ son las transformadas de Fourier de $g(t)$ y $f(t)$ respectivamente. En el lado derecho de (III-28), $t$ representa el instante en que interesa conocer, por ejemplo, la posición del oscilador y $t$ representa la historia del sistema durante la cual actúa la fuerza.

Como puede verse, la solución generalizada del problema del oscilador sigue paso a paso la teoría del análisis de Fourier, de modo que cada expresión matemática en este problema tiene una implicación física inmediata.

\section{d).- Analiticidad y Causalidad}

Substituyendo (III-21) en (III-27) se obtiene, para la función de Green del oscilador, la expresión

$$
g(\tau)=-\frac{1}{2 \pi} \int_{-\infty}^{\infty} \frac{e^{-i \omega \tau} d \omega}{\left(\omega-\omega_{1}\right)\left(\omega-\omega_{2}\right)}
$$

que puede ser evaluada en el plano complejo, en términos de la suma de los residuos del integrando en sus polos. Para ello se harán las siguientes consideraciones: La integral sobre el eje real en (III-29) puede generalizarse a dos integrales sobre contornos 
cerrados que contengan al eje real, una en el SPS y otra en el SPI de acuerdo con el intervalo de $\tau$ que se maneje. La generalización de (III-29) al resto del plano complejo es

$$
g(\tau)=-\frac{1}{2 \pi} \int_{c} \frac{e^{-i \omega \tau} d \omega}{\left(\omega-\omega_{1}\right)\left(\omega-\omega_{2}\right)}
$$

Antes de proceder a la integración de (III-30), es muy importante recordar que los polos en la descripción matemática de este sistema físico permanecen siempre en el SPI, independientemente del valor asumido por la constante de amortiguamiento $Y$ de acuerdo con la condición $y>0$. (cf Fig. (11-4).

Para ejecutar la integración de (III-30) se procede de la siguiente manera: Si $\tau=t-t<0$, es decir si "interesa" conocer la posición $x(t)$ del sistema antes de su excitación o dicho de otra manera, si "interesa" conocer el efecto antes de que ocurra la causa (lo cual es absurdo desde el punto de vista del principio de causalidad), entonces el contorno c es un semicírculo en el SPS que se cierra en el eje real. La integral sobre este contorno es equivalente a la integral sobre el eje real ya que la integral sobre el semicírculo se nulifica conforme su radio $R$ tiende a infinito, pues el comportamiento de la función exponencial en el integrando de (III-30) es

$$
e^{-i R(\operatorname{Cos} \theta+i \operatorname{Sen} \theta) \tau}=e^{-i R \tau \operatorname{Cos} \theta} e^{-R / \tau \mid \operatorname{Sen} \theta} \rightarrow 0 .
$$

Ahora bien, como $G(\omega)$ no tiene polos en SPS, entonces, por el teorema del residuo, la integración de (III-30) da cero y por lo tanto el principio de causalidad en este caso queda expresado matemáticamente como:

$$
g(\tau)=0 \quad, \quad \tau=t-t<0 .
$$

Esto es, la función de Green del sistema vale cero para tiempos anteriores a la excitación, o equivalentemente, la función de Green vale cero para esos tiempos si 
su transformada de Fourier es holomórfica, analítica o regular en el SPS y eje real. La causalidad se traduce matemáticamente en que $G(\omega)$ no tiene polos ni en el SPS ni en el eje real. Además, como $G(\omega)$ pertenece a la clase de funciones mencionadas en la Sección III-3, sus partes real e imaginaria están conectadas por la transformada de Hilbert y forman el par de relaciones de Kramers-Krönig. Por esto, también se dice que las relaciones de Kramers-Krönig son una representación matemática del principio de causalidad.

Para $\tau=t-t^{\prime}>0$, es decir, cuando interesa conocer la posición $x(t)$ del oscilador en algún instante posterior a la excitación o sea cuando interesa conocer el efecto después de la causa, el contorno $c$ se debe cerrar en el SPI. En este caso la integral sobre el eje real también es equivalente a la integral sobre el contorno $c$ ya que la integral sobre el semicírculo se nulifica conforme el radio de éste tiende a infinito:

$$
e^{-i R(\cos \theta-i \operatorname{Sen} \theta) \tau}=e^{-i R \tau \cos \theta} e^{-R|\tau| \operatorname{sen} \theta} \rightarrow 0 .
$$

En vista de que hay dos polos en el SPI, la integral (III-30) vale entonces

$$
g(\tau)=2 \pi i \sum_{i} \operatorname{Res}\left[\frac{G\left(\omega_{i}\right)}{2 \pi} e^{-i \omega_{i} \tau}\right], \quad \tau>0 . \quad(111-32)
$$

El signo menos, que cancela al de (III-30), se origina al recorrer el contorno $c$ en la dirección de giro de las manecillas del reloj. Empleando (III-16), la expresión (III-32) se puede reescribir como

$$
g(\tau)=\frac{e^{-\gamma \tau} \operatorname{Sen}\left[\left(\omega_{0}^{2}-\gamma^{2}\right)^{1 / 2} \tau\right]}{\left(\omega_{0}^{2}-\gamma^{2}\right)^{1 / 2}}, \quad \tau>0 . \quad \text { (III-33) }
$$

Finalmente al substituir en (III-26) la expresión matemática de la causalidad dada en (III-31) y la expresión (III-33), se obtiene la solución causal para el desplazamiento del oscilador bajo la acción de la fuerza $f(t)$ 


$$
x(t)=\int_{-\infty}^{t} \frac{e^{-\gamma\left(t-t^{\prime}\right)} \operatorname{Sen}\left[\left(\omega_{0}^{2}-\gamma^{2}\right)^{1 / 2}\left(t-t^{\prime}\right)\right]}{\left(\omega_{0}^{2}-\gamma^{2}\right)^{1 / 2}} f\left(t^{\prime}\right) d t \cdot(111-34)
$$

Nótese que la consecuencia de obedecer al principio de causalidad se refleja en que la integración de (III-34) se realiza sólamente desde el pasado remoto en que está actuando la fuerza que causa el movimiento, hasta el instante $t$ en el que interesa conocer el valor del desplazamiento. El efecto sigue a la causa.

El ejemplo expuesto refleja el hecho de que el principio de causalidad está intimamente ligado con la analiticidad de la transformada de Fourier de la función de Green asociada al problema, en el plano complejo de la frecuencia; en particular en el SPS y en el eje real $i$. e. la transformada no tiene polos ni en el SPS ni en el eje real.

Con el objeto de complementar el tema sobre analiticidad y causalidad $y$ en virtud de que el principio de causalidad puede ser representado matemáticamente por medio de las relaciones de Kramers-Krönig, en la siguiente sección se presentarán dos maneras alternativas y sencillas para obtener estas relaciones, una basada en el uso de la función escalón y la otra basada en el uso de la función signum.

\section{5.- DOS MANERAS ALTERNATIVAS Y SENCILLAS PARA OBTENER LAS RELACIONES DE KRAMERS-KRÖNIG}

\section{1- Uso de la función escalón}

Ben Yu-Kuang Hu [37] presenta una manera rápida y sencilla para obtener las relaciones de Kramers-Krönig basada en el empleo de:

a).- La transformada de Fourier de la función escalón. Esta última función es 


$$
\theta(t)=\left\{\begin{array}{ll}
1 & , t>0 \\
0 & , t<0
\end{array}\right\}
$$

b).- El teorema de convolución del análisis de Fourier. Este teorema está representado por la expresión (III-28) pero para los fines de esta sección, se escribirá como

$$
\int_{-\infty}^{\infty} f(t) g(t) e^{\imath \omega t} d t=\frac{1}{2 \pi} \int_{-\infty}^{\infty} F\left(\omega-\omega^{\prime}\right) G\left(\omega^{\prime}\right) d \omega \cdot .(111-36)
$$

Los aspectos anteriores se aplican a una señal lineal $\chi(t)$ con que responde un sistema a un estímulo. Como ésta debe ser una señal causal, es decir $\chi(l) \neq 0$ para $t>0$ y $\chi(t)=0$ para $t<0$, su forma debe ser la siguiente

$$
X(t)=\theta(t) Y(t)
$$

donde $Y(t)=\chi(t)$ para $t>0$. Para $t<0$ hay plena libertad en la elección de $Y(t)$ puesto que la presencia de la función $\theta(l)$, que vale cero para $t<0$, elimina cualquier tipo de función $Y(t)$ que se escoja para el intervalo $t<0$.

Es en este punto donde se propone una manera equivalente no arbitraria y más consistente que en [37] para elegir $Y(t)$ en el intervalo $t<0$. La elección consiste en definir $Y(t)=0$ para $t<0$. Una manera de lograr ésto es definir

$$
Y(t)=\frac{\chi_{p}(t)+x_{1}(t)}{2} \quad, \quad \forall t,
$$

donde $\chi_{P}(t)$ es una señal par con respecto a $t=0$ no causal, que se extiende desde $t=-\infty$ hasta $t=\infty$ pero tal que:

$$
\chi_{P}(t)=\chi(t), \text { para } t>0 .
$$

En (III-38) $\chi_{l}(t)$ es otra señal no causal, impar con respecto a $t=0$, construida de tal manera que 


$$
x_{1}(t)=x(t), \quad \operatorname{para} t>0, \quad(111-40)
$$

$y$ para $t<0, x_{1}(t)$ es el reflejo antisimétrico de $x(t)$. Las funciones $x_{p}(t)$ y $x_{1}(t)$ son tales que $\left|\chi_{P}(t)\right|=\left|\chi_{l}(t)\right|, \forall t$.

Con la anterior definición queda elegida $Y(t)$ de tal modo que ella misma es causal a pesar de estar compuesta de señales no causales y se comporta como

$$
Y(t)=\left\{\begin{array}{ll}
x(t) & , t>0 \\
0 & , t<0
\end{array}\right\},
$$

Esta elección es muy simple comparada con la de la Ref. [37] y como se verá, no sólo produce inmediatamente el resultado deseado sino que resalta la importancia capital de la función escalón en la definición de funciones causales.

Substituyendo (III-38) en (III-37), se obtiene

$$
x(t)=\frac{\theta(t) x_{p}(t)}{2}+\frac{\theta(t) x_{1}(t)}{2}, \forall t . \quad(111-42)
$$

Al aplicar la transformada de Fourier a (III-42), se llega a que

$$
\chi(\omega)=\frac{1}{2} \int_{-\infty}^{\infty} e^{i \omega t} \theta(t) \chi_{p}(t) d t+\frac{1}{2} \int_{-\infty}^{\infty} e^{i \omega t} \theta(t) \chi_{1}(t) d t \quad \cdot(I I I-43)
$$

Por el teorema de convolución (III-36), esta última expresión se çonvierte en

$$
\chi(\omega)=\frac{1}{4 \pi} \int_{-\infty}^{\infty} \Theta\left(\omega^{\prime}-\omega^{\prime}\right) x_{p}\left(\omega^{\prime}\right) d \omega^{\prime}+\frac{1}{4 \pi} \int_{-\infty}^{\infty} \Theta\left(\omega-\omega^{\prime}\right) x_{1}\left(\omega^{\prime}\right) d \omega^{\prime}
$$

Por otra parte, la función $\Theta(\omega)$, que es la transformada de Fourier de la función escalón [5], se puede escribir como el operador [13, p-416]

$$
\Theta(\omega)=\pi \delta(\omega)+i P \frac{1}{\omega}
$$


donde $P$ es el operador para tomar la parte principal [13]. Substituyendo (III-45) en (III-44), se tiene

$$
\chi(\omega)=\frac{i}{4 \pi} P \int_{-\infty}^{\infty} \frac{\chi_{P}\left(\omega^{\prime}\right) d \omega^{\prime}}{\omega-\omega^{\prime}}+\frac{\chi_{P}(\omega)}{4}+\frac{i}{4 \pi} P \int_{-\infty}^{\infty} \frac{\chi_{1}\left(\omega^{\prime}\right) d \omega}{\omega-\omega^{\prime}}+\frac{\chi_{1}(\omega)}{4} .
$$

$(I I I-46)$

Pero, de acuerdo con Ben Yu-Kuang [37], para una función par

$$
x_{P}(\omega)=2 \operatorname{Rex}(\omega)
$$

y para una función impar

$$
x_{1}(\omega)=2 i \operatorname{lm} x(\omega)
$$

Nótese que las expresiones (III-47) se deducen de (III-38) aplicando la transformada de Fourier a ambos miembros y considerando que la transformada de Fourier de una función par (impar) es puramente real (imaginaria).

Al substituir (III-47) en (III-46), se llega a

$\chi(\omega)=\frac{i}{2 \pi} P \int_{-\infty}^{\infty} \frac{\operatorname{Re\chi }\left(\omega^{\prime}\right) d \omega}{\omega-\omega^{\prime}}+\frac{\operatorname{Re\chi }(\omega)}{2}+\frac{i}{2 \pi} P \int_{-\infty}^{\infty} \frac{i \operatorname{lm} \chi\left(\omega^{\prime}\right) d \omega}{\omega-\omega^{\prime}}+\frac{i \operatorname{lm} \chi(\omega)}{2}$.

El lado izquierdo de (III-48) se puede descomponer en sus partes real e imaginaria y entonces la expresión se convierte en

$$
\operatorname{Rex}(\omega)+i \operatorname{Im} \times(\omega)=-\frac{1}{\pi} P \int_{-\infty}^{\infty} \frac{\operatorname{lm} \times\left(\omega^{\prime}\right) d \omega^{\prime}}{\omega-\omega^{\prime}}+i \frac{1}{\pi} P \int_{-\infty}^{\infty} \frac{\operatorname{Rex}\left(\omega^{\prime}\right) d \omega^{\prime}}{\omega-\omega^{\prime}},
$$

de donde se deducen inmediatamente las relaciones de Kramers-Krönig

$$
\operatorname{Rex}(\omega)=\frac{1}{\pi} P \int_{-\infty}^{\infty} \frac{\operatorname{lm} \times\left(\omega^{\prime}\right) d \omega}{\omega-\omega}
$$




$$
\operatorname{lm} x(\omega)=-\frac{1}{\pi} P \int_{-\infty}^{\infty} \frac{\operatorname{Rex}(\omega) d \omega}{\omega-\omega} .
$$

cuya forma coincide con la de las expresiones (III-7).

Con la elección (III-38) para $Y(t)$, las dos relaciones (III-50) se obtienen simultáneamente; en tanto que para obtenerlas segun Ben Yu-Kuang [37] es necesario hacer para $Y(t)$ una elección diferente para cada relación.

Es de hacer notar por otra parte, que para obtener las expresiones (III-50), tanto en la Ref. [37] como en esta presentación, fué determinante la presencia de la función escalón. Sin embargo en esta presentación donde se eligió $Y(t)=0$ para $t<0$, resalta más el papel que juega la función escalón en la causalidad. En efecto, las expresiones (III-50) no se hubieran podido obtener de no haberse introducido la función escalón la que, aparentemente, estaría desempeñando un papel redundante puesto que de todos modos $Y(t)=0$ para $t<0$. Sin embargo esta función escalón divide drásticamente el eje del tiempo en un "antes" y un "después" a partir de $t=0$.

Independientemente del método empleado para deducir las relaciones de Kramers-Krönig, ya sea sencillo o complicado, ningún autor, hasta donde el presente tiene conocimiento, hace resaltar la importancia de introducir la función escalón. En la deducción de estas relaciones se hace necesario introducir una condición matemática más rigurosa y formal que simplemente decir que tal o cual función vale cero para $t<0$. Esta condición debe tener la cualidad de llevar el mensaje substancial explícito de que el eje del tiempo queda dividido en un "antes" y un "después"; la función escalón juega este papel.

\section{2.- Uso de la función signum}

En esta sección se obtendrán las relaciones de Kramers-Krönig empleando la función signum en lugar de la función escalón. 
La siguiente información será de utilidad en el proceso

a) La transformada de Fourier de la función signum [34],

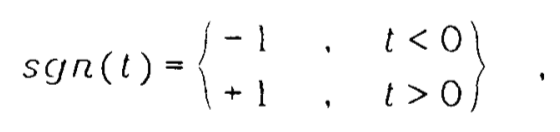

es

$$
\operatorname{SGN}(\omega)=i P \frac{2}{\omega}
$$

b) El teorema de convolución del análisis de Fourier está dado por

$$
\int_{-\infty}^{\infty} f(t) g(t) e^{i \omega t} d t=\frac{1}{2 \pi} \int_{-\infty}^{\infty} F\left(\omega-\omega^{\prime}\right) G\left(\omega^{\prime}\right) d \omega^{\prime} . \quad(I I I-52)
$$

donde $F(\omega)$ y $G(\omega)$ son las transformadas de Fourier de $f(t)$ y $g(t)$, respectivamente.

Esta información se aplicará ahora a una respuesta física o señal causal $\chi(t)$ que puede ser escrita como

$$
x(t)=\frac{x_{p}(t)+x_{1}(t)}{2}
$$

donde $\chi_{p}(t)$ es una función par del tiempo y es igual a $\chi(l)$ para $t>0 ; \chi_{1}(t)$ es una función impar del tiempo y es también igual a $\chi(l)$ para $t>0$.

Debido a que $x_{1}(t)$ puede ser escrita como

$$
X_{1}(t)=\operatorname{sgn}(t) X_{P}(t),
$$

la ecuación (III-53) se puede reescribir

$$
2 x(t)=x_{p}(t)+\operatorname{sgn}(t) x_{p}(t) .
$$


Antes de seguir adelante, es importante ver cómo la expresión (III-53) se puede representar en términos de la función $\theta(t)$ escalón. Para ello, la función $\operatorname{sgn}(t)$ se escribe en términos de $\theta(t)$ de la siguiente manera

$$
\operatorname{sgn}(t)=\theta(t)-\theta(-t)
$$

Nótese que, de acuerdo con (III-56), realizar la operación (III-54) es equivalente a introducir en (III-53) el "operador de causalidad" representado por la función escalón. Usando (III-56), la ecuación (III-55) se reescribe asi:

$$
2 x(t)=x_{p}(t)+\theta(t) x_{p}(t)-\theta(-t) x_{p}(t) .
$$

Es claro que $x_{p}(t)-\theta(-t) x_{p}(t)$ puede ser escrita como $\theta(t) x_{1}(t)$ ó $\theta(t) \chi_{p}(t)$. La primera elección permite escribir la ecuación (III-57) como

$$
2 x_{(t)}=\theta(t)\left[x_{p}(t)+x_{1}(t)\right] .
$$

Regresando a la ecuación (III-55), su transformada de Fourier está dada por

$$
2 \chi(\omega)=\chi_{p}(\omega)+\frac{1}{2 \pi} \int_{-\infty}^{\infty} S G N\left(\omega-\omega^{\prime}\right) x_{p}\left(\omega^{\prime}\right) d \omega^{\prime},
$$

o, usando (III-51),

$$
2 x(\omega)=x_{p}(\omega)+i \frac{1}{\pi} P \int_{-\infty}^{\infty} \frac{X_{P}(\omega) d \omega}{\omega-\omega}
$$

pero, de acuerdo con el libro de Bracewell [34]:

$$
\begin{array}{ll}
x_{p}(\omega)=2 \operatorname{Re}[x(\omega)], & (111-60 a) \\
x_{1}(\omega)=i 2 / m[x(\omega)] . & (111-60 b)
\end{array}
$$

Substituyendo (III-60a) en (III-59), se obtiene

$$
x(\omega)=\operatorname{Re}[x(\omega)]+i \frac{1}{\pi} p \int_{-\infty}^{\infty} \frac{\operatorname{Re}[x(\omega)] d \omega}{\omega-\omega}
$$

de donde se llega a la relación 


$$
\operatorname{lm}[x(\omega)]=-\frac{1}{\pi} P \int_{-\infty}^{\infty} \frac{\operatorname{Re}[x(\omega)] d \omega}{\omega-\omega}
$$

es decir, la parte imaginaria de $x(\omega)$ es la transformada de Hilbert, con signo negativo, de la parte real de $\chi(\omega)$.

Para obtener la otra relación de Kramers-Krönig nótese que, en analogia con la expresión (III-54), $\chi_{p}(t)$ puede ser escrita en términos de $x_{1}(t)$ y la función signum como

$$
X_{P}(t)=\operatorname{sgn}(t) X_{1}(t)
$$

Usando esta última definición, la ecuación (III-53) se puede expresar como

$$
2 \times(t)=\operatorname{sgn}(t) x_{1}(t)+x_{1}(t) .
$$

Aplicando la transformada de Fourier de (III-63), se llega a

$$
2 x(\omega)=\frac{1}{2 \pi} \int_{-\infty}^{\infty} \operatorname{SGN}\left(\omega-\omega^{\prime}\right) x_{1}\left(\omega^{\prime}\right) d \omega^{*}+x_{1}(\omega) .
$$

ó bien, usando (III-51)

$$
2 \chi(\omega)=\frac{i}{\pi} P \int_{-\infty}^{\infty} \frac{\chi_{1}\left(\omega^{\prime}\right) d \omega^{\prime}}{\omega-\omega}+\chi_{1}(\omega) .
$$

en la que, despues de substituir (III-60b), se obtiene

$$
x(\omega)=\frac{i}{\pi} P \int_{-\infty}^{\infty} \frac{i \operatorname{lm}\left[x\left(\omega^{\prime}\right)\right] d \omega^{\prime}}{\omega-\omega^{\prime}}+i \operatorname{lm}[x(\omega)]
$$

de donde surge la otra relación de Kramers-Krönig:

$$
\operatorname{Re}[x(\omega)]=\frac{1}{\pi} P \int_{-\infty}^{\infty} \frac{\operatorname{lm}\left[x\left(\omega^{\prime}\right)\right] d \omega^{\prime}}{\omega^{\prime}-\omega},
$$

En lo que sigue se analizarán los ejemplos mencionados en la introducción de este capítulo sobre formas aproximadas de la función de dispersión del plasma, considerando que los detalles y motivaciones para obtener las formas aproximadas 
en los tres primeros ejemplos, asi como las constantes involucradas, se encuentran en las referencias [30], [31], [32]. En el presente trabajo sólo interesa revisar si dichas formas aproximadas satisfacen las relaciones de Kramers-Krönig, pues ésto no fué realizado en esas referencias. Si alguna de las formas aproximadas que se revisarán no satisface estas relaciones, no puede ser considerada como relación de dispersión.

6.- PRIMER EJEMPLO: FORMA APROXIMADA DE LA FDP PROPUESTA POR B. D. FRIED et al. [30]

En este ejemplo se revisará una forma aproximada de la función de dispersión del plasma propuesta por Fried et al. [30], para determinar si sus partes real e imaginaria satisfacen las relaciones de Kramers-Krönig. Estos autores encuentran que su expresión aproximada

$$
\dot{Z}(\because)=\left[\frac{1}{A(A-\because)}-\frac{1}{A^{*}\left(A^{*}+\%\right)}\right](2 \xi)^{-1}, \quad(111-66)
$$

es muy buena en la mayor parte del semiplano superior (SPS). En esta expresión $z$, A y $\xi$ están definidas, respectivamente, como

$$
\begin{array}{cc}
\because=v+i a & \\
\frac{1}{A}=\xi+i \eta=0.55+i \frac{\sqrt{\pi}}{2} & (I I I-67)
\end{array}
$$

Con el objeto de analizar con comodidad la expresión (III-66), es conveniente racionalizarla y escribir por separado sus partes real e imaginaria. El resultado es $\operatorname{Re} \hat{Z}(v, \alpha)=-\frac{\alpha(u-\alpha)-\beta(\alpha-\beta)}{\left(\alpha^{2}+\beta^{2}\right)\left[(u-\alpha)^{2}+(\alpha-\beta)^{2}\right]}-\frac{\alpha(u+\alpha)+\beta(\alpha-\beta)}{\left(\alpha^{2}+\beta^{2}\right)\left[(u+\alpha)^{2}+(\alpha-\beta)^{2}\right]}, \quad \quad \quad(111-69 a)$ 
$\operatorname{Im} \hat{Z}(u, \alpha)=\frac{\beta(u-\alpha)+\alpha(\alpha-\beta)}{\left(\alpha^{2}+\beta^{2}\right)\left[(v-\alpha)^{2}+(\alpha-\beta)^{2}\right]}-\frac{\beta(u+\alpha)-\alpha(\alpha-\beta)}{\left(\alpha^{2}+\beta^{2}\right)\left[(u+\alpha)^{2}+(\alpha-\beta)^{2}\right]}$,

donde $\alpha$ y $\beta$ satisfacen la expresión $A=\alpha+i \beta$.

De inmediato se puede constatar que (III-69a,b) son, respectivamente, antisimétrica y simétrica con respecto a la variable $v$, lo cual concuerda con las propiedades de simetría de $Z(z)$ enunciadas en (II-44). También se puede comprobar fácilmente que la expresion (III-69a) es la transformada de Hilbert de (III-69b) y que ésta es igual a la transformada de Hilbert, con signo negativo, de (III-69a). En otras palabras, las formas aproximadas (III-69a,b) satisfacen las relaciones de Kramers-Krönig que son una representación matemática del principio de causalidad. En este sentido, las expresiones (III-69) forman parte de una relación de dispersión y desde un punto de vista formal, se les puede aplicar en espectroscopia y en problemas de dispersión.

De (III-69a, b) se pueden obtener expresiones aproximadas para los perfiles de Voigt y de dispersión, usando (II-43a,b), respectivamente.

Nótese que (III-69a, b) tienen una naturaleza básicamente lorentziana lo cual, de acuerdo con la afirmación hecha por Fried et al. [30] acerca de que (III-66) es buena en la mayor parte del SPS, significa que en términos del parámetro a, la expresión (III-66) puede ser usada en el intervalo $a>1$. Recuérdese que a es proporcional a la razón de la anchura lorentziana y a la anchura gaussiana (cf. ec (II-4b)). Dicho de otra manera: las expresiones (III-69a, b) guardan poca memoria acerca de la componente gaussiana de $Z(z)$; ésto quiere decir que las expresiones (III-69a, b) sólo pueden ser usadas para ajustar perfiles experimentales de los cuales se sepa previamente que tienen componente gaussiana muy pequeña. En la práctica las expresiones (III-69a, b) son útiles para casos en los que $a \gg 1$. 


\section{7.- SEGUNDO EJEMPLO: FORMA APROXIMADA DE LA FDP PROPUESTA POR MARTIN Y GONZALEZ [31]}

Al igual que en el ejemplo anterior, se analizará ahora la forma aproximada para la función de dispersión del plasma propuesta por Martín y González [31] para revisar si cumple con las relaciones de Kramers-Krönig. Estos autores encuentran que su expresión aproximada

$$
\hat{Z}(\because)=\frac{b}{(1-\because)}-\frac{b^{*}}{\left(t^{*}+z\right)} \quad, \quad(111-70)
$$

es buena en la mayor parte del semiplano superior. En (III-70), las tres literales $\approx, 4$ y $b$ representan a las expresiones $z=v+i c, \quad A=\gamma+i \epsilon=0.5138-i 1.0324 \mathrm{y}$ $b=\alpha+i \beta=0.5+i 1.2891$.

Racionalizando (III-70) y escribiendo separadamente sus partes real e imaginaria se obtiene

$$
\begin{aligned}
& \operatorname{Re} \hat{Z}(v, a)=-\frac{\alpha(v-\gamma)+\beta(a-\epsilon)}{(v-\gamma)^{2}+(a-\epsilon)^{2}}-\frac{\alpha(u+\gamma)-\beta(a-\epsilon)}{(v+\gamma)^{2}+(a-\epsilon)^{2}}, \quad(11 /-71 a) \\
& \operatorname{Im} \hat{Z}(v, a)=\frac{-\beta(u-\gamma)+\alpha(a-\epsilon)}{(v-\gamma)^{2}+(a-\epsilon)^{2}}+\frac{\beta(v+\gamma)+\alpha(a-\epsilon)}{(v+\gamma)^{2}+(a-\epsilon)^{2}}, \quad(11 /-71 b)
\end{aligned}
$$

De inmediato se puede constatar que las expresiones (III-71) son, respectivamente, antisimétrica y simétrica con respecto a la variable $v$, lo cual concuerda con las propiedades de simetria de $Z(z)$ enunciadas en (II-44). Tambien se puede comprobar que la expresión (III-71a) es la transformada de Hilbert de (III-71b) y que ésta es igual a la transformada de Hilbert, con signo negativo, de (III-71a). Esto significa que las formas aproximadas (III-71a, b) satisfacen las relaciones de Kramers-Krönig. Como en el ejemplo anterior, la naturaleza de las expresiones (III-71) es básicamente Lorentziana y por lo tanto útil para situaciones en las que $a>1$. 


\section{8.- TERCER EJEMPLO: FORMA APROXIMADA DE LA FDP PROPUESTA POR ROBINSON AND NEWMAN [32]}

Como en los ejemplos anteriores se procederá ahora a analizar la forma aproximada presentada por Robinson y Newman en la referencia [32]. Estos autores presentan la siguiente forma aproximada para la FDP

$$
\hat{Z}(z)=\frac{i \sqrt{\pi}+p z}{1-i \sqrt{\pi} z+p z^{2}} .
$$

donde $z=v+i a$ y $p$ es un parámetro adimensional. Los autores analizan los casos para $p=0,2, \pi-2$. La racionalización de (III-72) produce las expresiones

$$
\begin{aligned}
& \operatorname{Re} \hat{Z}(u, a)=\frac{\left(p K_{1}-B K_{2}\right) u-p^{2} u^{3}}{\left(K_{1}-p u^{2}\right)^{2}+K_{2}^{2} u^{2}}, \\
& \operatorname{Im} \hat{Z}(u, a)=\frac{B K_{1}+\left(p K_{2}-B p\right) u^{2}}{\left(K_{1}-p v^{2}\right)^{2}+K_{2}^{2} u^{2}},
\end{aligned}
$$

donde

$$
K_{1}=1+\sqrt{\pi} a+p a^{2}, \quad K_{2}=\sqrt{\pi}+2 p c t, \quad B=\sqrt{\pi}+p a t .
$$

Los autores de la referencia [32] encuentran que el mejor ajuste de (III-73) a la forma exacta de $Z(z)$, se tiene para $p=\pi-2$. Sin embargo del análisis de las expresiones (III-73) se deduce que sólo para el caso $p=0$ se dispone de un par que satisface las relaciones de Kramers-Krönig. Este par es

$$
\operatorname{Re} \hat{Z}(v, a)=-\sqrt{\pi} \frac{v}{v^{2}+\frac{(1+\sqrt{\pi} a)^{2}}{\pi}} \quad, \quad(1 / I-74 a)
$$




$$
\operatorname{Im} \hat{Z}(v, a)=(1+\sqrt{\pi} a) \frac{1}{u^{2}+\frac{(1-\sqrt{\pi} a)^{2}}{\pi}} \quad, \quad(I I I-74 b)
$$

el cual, por otra parte, ofrece un ajuste bastante malo a la forma exacta de $Z(z)$ como puede ser constatado en la Ref. [32]. La expresión (III-74b) es un perfil lorentziano y (III-74a) su transformada de Hilbert. Se puede observar también que la naturaleza de este par es totalmente lorentziana independientemente del valor de $a$. Es necesario recordar que un valor pequeño de a implica una pequeña contribución lorentziana al perfil de Voigt y que un valor grande de a una pequeña contribución gaussiana. Por lo tanto para tener congruencia entre la forma de (III-74) y el valor de $a$. esta forma de perfil sólo se acomoda a un valor muy grande de a icontribución gaussiana despreciable). En este caso el par (III-74) se convierte en

$$
\begin{aligned}
& \operatorname{Re} \hat{Z}(v, a)=-\sqrt{\pi} \frac{v}{v^{2}+a^{2}} \quad a \gg 0, \quad(111-75 a) \\
& \operatorname{lm} \hat{Z}(v, a)=\sqrt{\pi} \frac{a}{v^{2}+a^{2}} \quad a \gg 0
\end{aligned}
$$

Del análisis de este ejemplo se concluye que la aproximación presentada por Robinson y Newman [32], es aceptable para el caso $p=0$, mala para los casos $p=2, \pi-2$ y adecuada solamente para ajustar espectros experimentales con contenido gaussiano despreciable.

Antes de presentar un cuarto ejemplo en el que se propone una forma sencilla de obtener un nuevo par absorción-dispersión, que cumple con las relaciones de Kramers-Krönig, es pertinente mencionar que existe otra razón práctica para insistir en la necesidad de que las partes real e imaginaria de una relación que se pretende sea de dispersión, estén conectadas por estas relaciones. La razón es que cumpliendo con esa exigencia, las partes real e imaginaria de la expresión considerada pueden ser 
usadas para construir gráficas "DISPA" (DISPersión-Absorción) [17, 38, 39, 40], las cuales proporcionan información acerca de la desviación de los espectros experimentales del perfil lorentziano. En un capitulo posterior se tratará con más amplitud este tema y se propondrá por parte de este autor y otros, un nuevo método para deconvolver líneas espectrales con la ayuda de datos y gráficas DISPA, para el caso en que dichas líneas satisfagan la condición $a \gg 1 / \sqrt{2}$.

\section{9.- CUARTO EJEMPLO: FORMA APROXIMADA DE LA FDP PROPUESTA EN ESTE TRABAJO}

En este ejemplo se propone, un par absorción-dispersión útil en espectroscopía para ser usado en los casos de pequeña contribución gaussiana. Se trata de un ejemplo muy simple en el que el desarrollo no se basa en la función de dispersión del plasma. Se basa en la aplicación de la transformada de Hilbert a un perfil de Voigt aproximado, propuesto por Flores-LLamas et al., [33], que es válido en el intervalo $a \gg 1 / \sqrt{2}$. El par absorción-dispersión puede ser usado para ajustar espectros experimentales en los que las líneas espectrales pueden ser descritos por perfiles del tipo voigtiano. Esto último ocurre en muchos caso de interés espectroscópico pero también, como se ha visto en el Capítulo II, para estudiar el espectro de frecuencias de ondas que se propagan en un plasma maxwelliano.

El perfil aproximado de absorción de Voigt propuesto por Flores-LLamas et al. [33], cuya deducción será glosada en el capítulo IV, es

$$
H_{A P R}(v, a)=\frac{a}{\sqrt{\pi}} \frac{1}{v^{2}+a^{2}}+\frac{a}{2 \sqrt{\pi}} \frac{3 v^{2}-a^{2}}{\left(v^{2}+a^{2}\right)^{2}}, \quad(111-76)
$$


donde $v, a$ están dadas por las expresiones adimensionales definidas en el capítulo II, transcritas aqui para comodidad del lector.

$$
\begin{gathered}
u=\frac{2\left(v-v_{0}\right)}{\Gamma_{G}}, \overline{\ln 2}, \\
a=\frac{\Gamma_{L}}{\Gamma_{G}} \sqrt{\ln 2},
\end{gathered}
$$

cuyos significados fueron establecidos en ese capítulo.

Para encontrar la expresión del perfil de dispersión que corresponde unívocamente al de absorción (III-76) se usa el hecho de que para un sistema causal en ausencia de efectos no lineales, los fenómenos de absorción y dispersión están conectados por las relaciones de Kramers-Krönig, es decir, absorción y dispersión son la transformada de Hilbert una de la otra. En el presente ejemplo la expresión (III-76) puede ser escrita como

$$
H_{A P R}(u, a)=\frac{a}{\sqrt{\pi}}\left[\frac{1}{v^{2}+a^{2}}\right]+\frac{a}{4 \sqrt{\pi}} \frac{\partial^{2}}{\partial v^{2}}\left[\frac{1}{v^{2}+a^{2}}\right] \cdot \quad(1 / I-77)
$$

El resultado de obtener la transformada de Hilbert [41] de (III-77) provee el perfil de dispersión dado por

$$
D_{A P R}(v, a)=-\frac{1}{\sqrt{\pi}}\left[\frac{v}{v^{2}+a^{2}}\right]-\frac{1}{2 \sqrt{\pi}}\left[\frac{v\left(v^{2}-3 a^{2}\right)}{\left(v^{2}+a^{2}\right)^{2}}\right] \cdot(111-78)
$$

Esta expresión es válida también en el intervalo $a \gg 1 / \sqrt{2}$. Para los fines de este trabajo conviene enfatizar que el resultado (III-78) es inédito y ha sido publicado en la Ref. [7] durante el desarrollo de esta tesis. Se trata del perfil de dispersión que forma pareja con el de absorción dado por la expresión (III-77). El par (III-76), (III-78) será motivo de estudio adicional y de uso práctico en el capítulo IV. 
De acuerdo con las relaciones (II-43) las correspondientes expresiones para las partes real e imaginaria de la función de dispersión del plasma, $Z(z)$, son

$$
\begin{array}{ll}
\operatorname{Re}[Z(\because)]=-\frac{v}{v^{2}+a^{2}}-\frac{1}{2}\left[\frac{v\left(v^{2}-3 a^{2}\right)}{\left(v^{2}+a^{2}\right)^{2}}\right] . & (111-79 a) \\
\operatorname{Im}[Z(z)]=\frac{a}{v^{2}+a^{2}}+\frac{a}{2}\left[\frac{3 v^{2}-a^{2}}{\left(v^{2}+a^{2}\right)^{2}}\right], & (11 I-79 b)
\end{array}
$$

ambas válidas en el intervalo $a \gg 1 / \sqrt{2}$. Estos valores de a implican que las expresiones (III-79) pueden ser usadas para estudiar el espectro de frecuencias de ondas electrostáticas que se propaguen en un plasma maxwelliano tibio. En efecto, el ancho gaussiano $\Gamma_{G}$. implícito en los perfiles (III-79) y que se encuentra en el denominador de $a$, es proporcional a la temperatura electrónica del plasma como fué indicado en el Capítulo II; si esta temperatura es suficientemente baja, el valor de a puede hacerse mucho más grande que $1 / \sqrt{2}$. Tambien puede comprobarse fácilmente que las expresiones (III-79) satisfacen las condiciones de simetría dadas en (II-44).

Los anteriores cuatro ejemplos han servido para ilustrar el manejo de las partes (absorción y dispersión) que componen a una relación de dispersión y su uso potencial en espectroscopia. Ahí pueden ser usadas para el ajuste de perfiles espectrales experimentales de absorción y/o dispersión para la determinación de parámetros espectrales de interés y para la simulación de espectros. En el cuarto ejemplo se ha mostrado cómo a partir de un perfil de absorción dado se puede obtener su complemento que es el perfil de dispersión y así construir una relación de dispersión que puede tener utilidad en espectroscopía.

En los capítulos siguientes se presentarán algunas aplicaciones prácticas del empleo del par absorción-dispersión, asi como de datos y gráficas DISPA. Se presentarán también nuevos métodos y modelos de linea para el análisis de espectros en varias espectroscopías, basados en la información presentada hasta ahora. 


\section{CAPITULO IV}

\section{UN MODELO CON ASIMETRIA DEL PAR ABSORCION-DISPERSION \\ PARA EL CASO $a \gg 1 / \sqrt{2}$}

\section{1.- INTRODUCCION}

En el capítulo anterior se presentaron los conceptos básicos que sirven para sustentar las relaciones de dispersión que están compuestas por un perfil de absorción y uno de dispersión. Se presentaron también varios ejemplos de la literatura sobre formas aproximadas de la función de dispersión del plasma para revisar si ellas cumplían con las condiciones de simetría que se imponen en general a las relaciones de dispersión. Al final se desarrolló otro ejemplo que consiste en un modelo de absorción-dispersión desarrollado por este autor y otros que servirá de base para el tema que se tratará en este capítulo. Aqui se enfatizará nuevamente la aplicación de esos modelos a la espectroscopía en general.

En este capítulo los perfiles voigtianos aproximados (III-76) (III-78) para absorción y dispersión respectivamente, serán considerados con más amplitud. En primer lugar se demostrará que la combinación adecuada de esos perfiles reproduce los dos primeros términos del desarrollo asintótico de la FDP. También se mostrará la manera de obtener los términos restantes de ese desarrollo. Enseguida se procederá a generalizar los perfiles de absorción-dispersión por medio de la inclusión de un parámetro de asimetría, con el objeto de aplicarlos en la simulación y/o ajuste de líneas espectrales asimétricas cuya aparición es muy frecuente en diversas espectroscopías.

Es necesario advertir que no se propondrá un significado físico del parámetro de asimetría ya que la asimetría de líneas espectrales puede deberse a distintas causas en las diversas espectroscopías. 
Finalmente, el perfil de absorción aproximado (caso $a \gg 1 / \sqrt{2}$ ) y caracterizado también ahora por un factor adimensional de asimetría $b$. será aplicado a un problema experimental. El problema consiste en determinar la temperatura de la componente neutra de un plasma de helio producido por resonancia ciclotrónica de electrones (RCE).

Parte del material presentado en este capítulo fué publicado en la referencia [7].

\section{2.- CONEXION ENTRE LOS PERFILES $H_{A P R}(u, a), D_{A P R}(u, a)$ Y LA FUNCION DE DISPERSION DEL PLASMA}

A continuación se verá cómo, partiendo de las expresiones (III-76) y (III-78) se pueden obtener los dos primeros términos del desarrollo asintótico de la función de dispersión del plasma (FDP). Esto mostrará la intima relación que existe entre la integral de Voigt obtenida a principios de siglo [3] y la FDP obtenida posteriormente [4]. Para realizar lo anterior, se usa la siguiente relación (II-43a)

$$
\text { Im } Z(z)=\sqrt{\pi} H(u, a), \quad(I V-1)
$$

donde $z$ está dada por (II-11) [19]. Análogamente, la transformada de Hilbert de (IV-1) relaciona la parte real de $Z(z)$ con el perfil de dispersión $D(v, a)$ (II-43b)

$$
\operatorname{Re} Z(z)=\sqrt{\pi} D(v, a) . \quad(I V-2)
$$

Combinando (IV-1) y (IV-2) se obtiene

$$
Z(z)=\sqrt{\pi}[D(u, a)+i H(u, a)] .
$$

La expresión (IV-3) es la función de dispersión del plasma escrita en términos de los perfiles espectrales de absorción y dispersión. Substituyendo las expresiones (III-76) y (III-78) en (IV-3) y haciendo un poco de álgebra se obtiene 


$$
Z_{A P R}(\because)=-\frac{1}{\because}-\frac{1}{2 \because^{3}}, a \gg 1 / \sqrt{\pi}
$$

Es fácil comprobar que esta última expresión satisface las condiciones de simetría para la FDP dadas en (II-44). Se observa también que (IV-4) está compuesta por los dos primeros términos del desarrollo asintótico de $Z(z)$ para el caso de $I m z>0$, [4] $y$ es posible agregar términos adicionales a (IV-4) mediante el siguiente procedimiento:

1.- De acuerdo con Flores-LLamas et al. [33], dadas dos funciones arbitrarias bien comportadas $r(y)$ y $f(y)$, su convolución

$$
h(u)=\int_{-\infty}^{\infty} r(u-y) f(y) d y \text {. }
$$

puede escribirse por medio del siguiente desarrollo en serie alrededor de $y=v$

$$
h(u)=C_{o} f(u)+\sum_{n=1}^{\infty} C_{n} \frac{f^{(n)}(v)}{n !} .
$$

donde $f^{(n)}(u)$ denota la enésima derivada de $f(v)$ y los coeficientes $C_{n}$ están dados por

$$
C_{n}=\int_{-\infty}^{\infty} \theta^{n} r(\theta) d \theta
$$

$\operatorname{con} \theta=v-y$. Continuando el procedimiento, las expresiones (IV-6) y (IV-7) son aplicadas a las siguientes funciones lorentziana y exponencial, respectivamente

$$
f(\theta)=\frac{1}{v^{2}+a^{2}}
$$

$y$

$$
r(\theta)=\exp \left(-\theta^{2}\right)
$$

de tal manera que es posible ir agregando términos adicionales a (III-76). 
2.- La transformada de Hilbert de esos nuevos términos se agrega a (III-78).

3.- Las nuevas expresiones para $H_{A P R}(u, a)$ y $D_{A P R}(u, a)$ que tienen un término más cada vez, se combinan de acuerdo con (IV-3).

4.- De esta forma se van agregando nuevos términos a (IV-4) y cada nueva expresión para $Z_{A P R}(\ddot{*})$ obtenida en la forma indicada, satisface las condiciones de simetría para la FDP dadas por (II-44).

5.- Procediendo en esta forma se puede obtener la expresión

$$
Z_{A P R}(z)=-\sum_{n=0}^{\infty} \frac{1}{\sqrt{\pi}}\left(n-\frac{1}{2}\right) ! z^{-(2 n+1)},
$$

la cual coincide con la del desarrollo asintótico de la FDP para $/$ lm $::>0$, reportada en la referencia [4].

La expresión (IV-10) puede ser de utilidad en espectroscopía para simular o ajustar líneas espectrales voigtianas ya que ella contiene, a traves de la variable compleja $z$, las anchuras lorentziana $\Gamma_{L}$ y gaussiana $\Gamma_{C}$ de los correspondientes perfiles convolucionados en dichas líneas.

Es necesario hacer notar que el perfil voigtiano de absorción aproximado dado por la expresión (III-76) fué obtenido en la referencia [33] por medio del desarrollo en serie indicado en la expresión (IV-6). Sin embargo, no es obvio de este resultado que el desarrollo asintótico de $Z(\because)$ se obtenga como una consecuencia inmediata. En efecto, como se ha mostrado aqui, la FDP es obtenida por medio de la transformada de Hilbert del perfil voigtiano realizando de esta manera la extensión de éste al resto del plano complejo $z$.

Es claro ahora que tanto la expresión exacta de la FDP como sus formas aproximadas que aparecen en la literatura, por ejemplo en las referencias [30-32], pueden ser usadas para analizar perfiles voigtianos de absorción y dispersión en varias espectroscopías. Además, como se ha mostrado aqui, no es necesario generar expresiones aproximadas para el perfil de Voigt por medio de (IV-6) con el objeto de 
obtener mejores aproximaciones para $\Gamma_{L}$ y $\Gamma_{G}$ ya que solamente se requiere tomar de (IV-10) el número suficiente de términos para obtener la aproximación deseada de los parámetros $\Gamma_{L}$ y $\Gamma_{G}$ en el análisis de datos experimentales de absorción y/o dispersión y separar las partes real e imaginaria en esos términos (cf. ecs. IV-1 y IV-2). Los valores de $\Gamma_{L}$ y $\Gamma_{G}$ se obtienen entonces a través de un proceso de ajuste de las expresiones analíticas $Z(\because) \circ Z_{A P R}(:)$ a un espectro experimental dado.

\section{3.- GENERALIZACION DE LOS PERFILES ESPECTROSCOPICOS $H_{A P R}(u, a)$ y $D_{A P R}(u, a)$ PARA INCLUIR UN PARAMETRO DE ASIMETRIA}

Debido a que muchas líneas en las diferentes espectroscopías presentan algún grado de asimetría, es conveniente obtener una generalización de las expresiones (III-76) y (III-78) que permita emular líneas asimétricas. Por ello en esta sección se introducirá un parámetro adimensional de asimetría en esas expresiones, de acuerdo con una idea parecida a la manejada por Losev [42]. Sin embargo, no se asignará una interpretación física particular a ese parámetro debido a que la asimetría puede tener diferente origen en las distintas espectroscopias. El parámetro de asimetría se introducirá primero en el perfil de absorción y después se obtendrá el perfil de dispersión tomando la transformada de Hilbert del primero. La forma del perfil de absorción dada por (III-77) se presta para realizar la operación notando que ésta se puede reescribir como

$$
H_{A P R}(v \cdot a)=\frac{a}{\sqrt{\pi}}\left[1+\frac{1}{4} \frac{\partial^{2}}{\partial v^{2}}\right] f(v \cdot a) \text {. }
$$

donde $f(u, a)$ está dada por la lorentziana (IV-8). Se pueden agregar términos adicionales dentro del paréntesis cuadrado de (IV-11) para los cuales los coeficientes se obtienen usando la fórmula (IV-7). Nótese que de acuerdo con (IV-7), los operadores diferenciales parciales que se vayan agregando en (IV-11) serán todos de orden par. 
La generalización se hará de la manera más simple posible, a saber, reemplazando la expresión (IV-8) por la siguiente expresión asimétrica

$$
f_{\sin u(u, a)}=\frac{\exp (b v)}{u^{2}+a^{2}} .
$$

en la cual el factor adimensional de asimetría $b$ puede tomar un conjunto de valores contenido en un intervalo que será determinado posteriormente. El nuevo perfil de absorción se describe de acuerdo con la expresión

$$
H_{A \operatorname{SiM}}(u, a)=\frac{a}{\sqrt{\pi}}\left[1+\frac{1}{4} \frac{\partial^{2}}{\partial u^{2}}\right] f_{A \operatorname{SiM}}(v, a) \text {. }
$$

y el perfil de dispersión se obtiene tomando la transformada de Hilbert $(T H)$ de (IV-13)

$$
D_{A \operatorname{SIM}}(v, a)=\frac{a}{\sqrt{\pi}}\left[1+\frac{1}{4} \frac{\partial^{2}}{\partial v^{2}}\right](T H) f_{A \operatorname{SIM}}(v, a) .
$$

La transformada de Hilbert de la expresión (IV-14) involucra la siguiente operación

$$
\frac{1}{\pi} P \int_{-\infty}^{\infty} \frac{f\left(x^{2}\right) d x}{x^{2}-x}
$$

donde el símbolo $P$ significa que debe tomarse la parte principal de la integral.

La integral de (IV-15) aplicada a la función (IV-12) puede ser realizada en el plano complejo eligiendo adecuadamente un contorno de integración. La elección de este contorno será sugerida por el comportamiento del integrando de (IV-15), después de haber sustituido en él la expresión (IV-12). La integral resultante es

$$
\int_{c} \frac{\exp (b z) d z}{(z-v)(z+i c t)(z-i c l)}
$$

donde la variable $v$ y los parámetros $a, b$ son reales. Se observa en esta expresión que los polos del integrando son $z=u$, sobre el eje real; $z_{-}=i a$ y $z_{-}=-i a$, sobre el eje imaginario, todos ellos de primer orden. Considerando la forma del integrando, se elegirá un contorno de integración como el de la Fig. III-3, pero en el caso presente 
se excluirá el polo sobre el eje real. La justificación para excluir este polo se dará más adelante. Para analizar el comportamiento del integrando de (IV-15), después de substituir (IV-12), se observa que si se escribe la variable de integración como $z=R \exp (i \theta)$, y se pemite que el radio $R$ de ese círculo crezca indefinidamente, la integral sobre el contorno circular no diverge $y$ puede incluso nulificarse con tal de que se imponga sobre el parámetro $b$, la siguiente condición:

$$
0 \leq b<1 \text {. }
$$

con el objeto de impedir que el integrando crezca indefindamente cuando $x \rightarrow \infty$.

En estas condiciones y en el limite cuando el radio de la pequeña circunferencia que rodea al polo $:=v$ tiende a cero, la integral considerada asume la forma simbólica (ver la Fig. 3 pero con el polo sobre el eje real excluído de tal manera que el pequeño contorno alrededor de este polo se recorre en el sentido del giro de las manecillas del reloj)

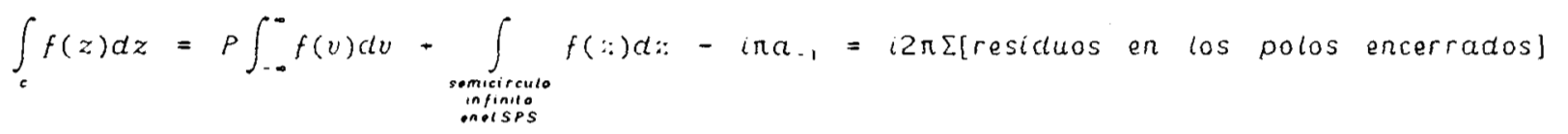

Donde $a_{-1}$ es el resíduo del integrado en el polo excluido $:=v$ y tiene un signo negativo ya que el pequeño contorno se recorre en el sentido del giro de las manecillas del reloj. Tomando los resíduos en los polos considerados $(z=v, z=i a)$ (el primero excluido y el segundo incluido) y después de un poco de álgebra se encuentra que la integral considerada en (IV-15) y aplicada a (IV-12), proveé la siguiente expresión compleja

$$
\begin{aligned}
(T H) f_{A S I M}(v, a) & =-\frac{1}{a} \frac{v \operatorname{Cos}(a b)-a \operatorname{Sen}(a b)}{v^{2}+a^{2}}+ \\
& +i\left[\frac{\exp (b u)}{v^{2}+a^{2}}-\frac{1}{a} \frac{a \operatorname{Cos}(a b)+v \operatorname{Sen}(a b)}{v^{2}+a^{2}}\right], \quad 0 \leq b<1 \quad(I V-17)
\end{aligned}
$$


En vista de que la parte principal de la integral en (IV-15) es una cantidad real, la transformada de Hilbert de (IV-12) es la parte real de (IV-17).

Obsérvese en (IV-17) que, para $b=0$ (el caso de un perfil simétrico), se cancela la parte imaginaria y se recobra el primer término de la expresión (III-78). Además, si se efectúan las operaciones para obtener la transformada de Hilbert de (IV-17), se obtiene de nuevo la expresión (IV-12). Es fácil demostrar que esto no hubiera podido lograrse de haber incluido el polo $z=v$. sobre el eje real, en el contorno de integración para la integral (IV-15). En efecto, la inclusión del polo $z=v$, en el contorno de integración implica un cambio de signo que impediría la mencionada cancelación de la parte imaginaria cuando $b=0$. Esta es la justificación para excluir ese polo del contorno de integración para (IV-15).

Antes de iniciar un análisis de la expresión (IV-17), es necesario establecer que, para que la expresión (IV-12) pueda ser usada como punto de partida para obtener un perfil útil para el ajuste de líneas espectrales, debe tener un máximo. Efectuando la operación de derivada de la expresión (IV-12), se encuentra que ésta puede tener máximos sólo si se cumple la siguiente condición sobre los parámetros $a$ y $b$ :

$$
a^{2} b^{2} \leq 1 \text {. }
$$

Por otra parte, se ha enfatizado que el modelo de absorción-dispersión basado en la expresión (III-77) se aplica solamente si se cumple la condición $a \gg \frac{1}{\sqrt{2}}$. Tomando en cuenta ésto, se encuentra que la condición más general y restrictiva sobre el parámetro de asimetría $b$ es que

$$
|b| \ll 1 .+1+2 \text {. }
$$

Los valores que el parámetro de asimetria $b$ toma en este intervalo, determinan la forma del perfil de acuerdo con el siguiente esquema: Para los valores $b>0$, se tienen líneas con asimetría del lado derecho; la condición $b=0$ provee una línea sin asimetría; en tanto que $b<0$, da líneas con asimetría del lado izquierdo. 
Si se realiza un analisis cuidadoso del comportamiento de las partes real e imaginaria de la expresión (IV-17) se concluye que los valores de la parte imaginaria son muy pequeños comparados con los de la parte real en un intervalo de valores de u suficientemente amplio como para explorar y ajustar una linea experimental. Como consecuencia de este análisis, se propone para aplicaciones prácticas el siguiente intervalo de valores para $b$.

$$
|6| \leq 0.08
$$

Finalmente, para obtener el par absorción-dispersióncon asimetría, se substituye la expresión (IV-12) en (IV-13) y la parte real de (IV-17) en (IV-14). Se llega entonces a las siguientes expresiones para los perfiles asimétricos de absorción y dispersión

$$
\begin{aligned}
& H_{A \operatorname{SIM}}(u, a, b)=\frac{a}{\sqrt{\pi}} \frac{\exp (b u)}{u^{2}+a^{2}}+\frac{a \exp (b u)}{4 \sqrt{\pi}}\left[\frac{b^{2}\left(v^{2}+a^{2}\right)^{2}-+b u\left(v^{2}+a^{2}\right)+2\left(3 v^{2}-a^{2}\right)}{\left(v^{2}+a^{2}\right)^{3}}\right] \\
& (I V-21) \\
& D_{A \sin }(v, a, b)=\frac{-1}{\sqrt{\pi}} \frac{v \operatorname{Cos}(a b)-a \operatorname{Sen}(a b)}{v^{2}+a^{2}}-\frac{1}{2 \sqrt{\pi}}\left[\frac{a^{3} \operatorname{Sen}(a b)+v\left(v^{2}-3 a^{2}\right) \operatorname{Cos}(a b)-3 a v^{2} \operatorname{Sen}(a b)}{\left(v^{2}+a^{2}\right)^{3}}\right] \\
& (I V-22)
\end{aligned}
$$

donde:

$$
a \gg \frac{1}{\sqrt{2}},|b| \leq 0.08
$$

Las Figuras IV-1 A, B muestran las gráficas correspondientes a las expresiones (IV-21) y (IV-22) para $a=6$ y $b=0 . \pm 0.018$, como ejemplos en los que las propiedades de asimetría inducidas por el parámetro $b$, se manifiestan con claridad. 


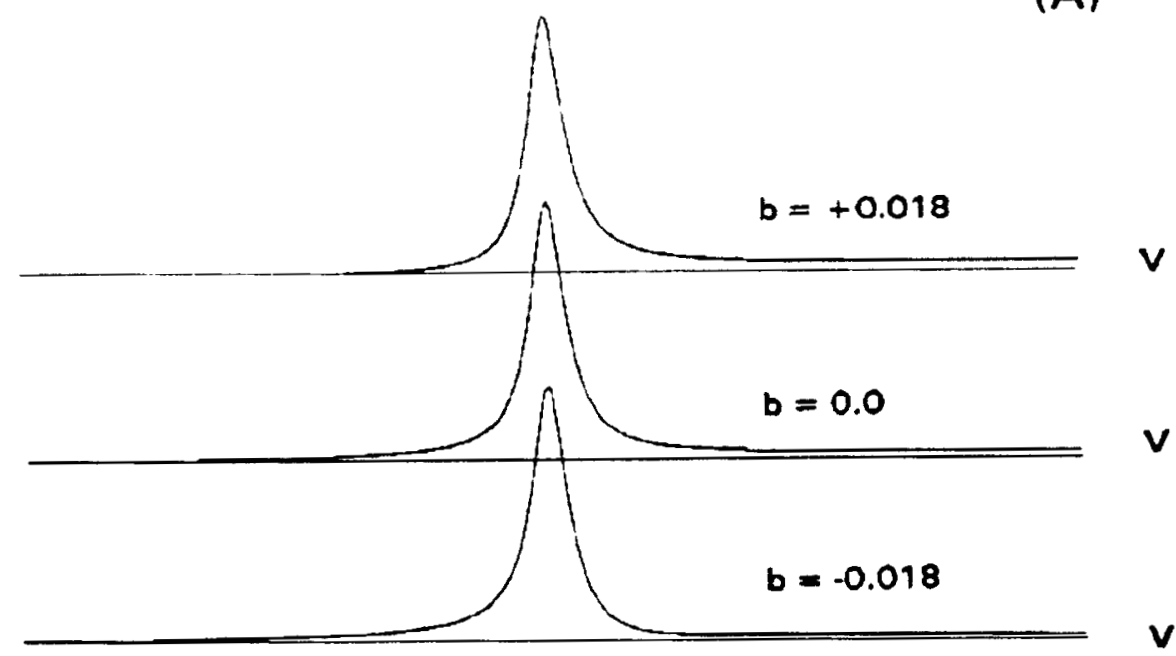

Figura IV-1.- Formas de línea de absorción representadas por la expresión (IV-21) para $a=6$ y $b=0, \pm 0.018$.

(B)

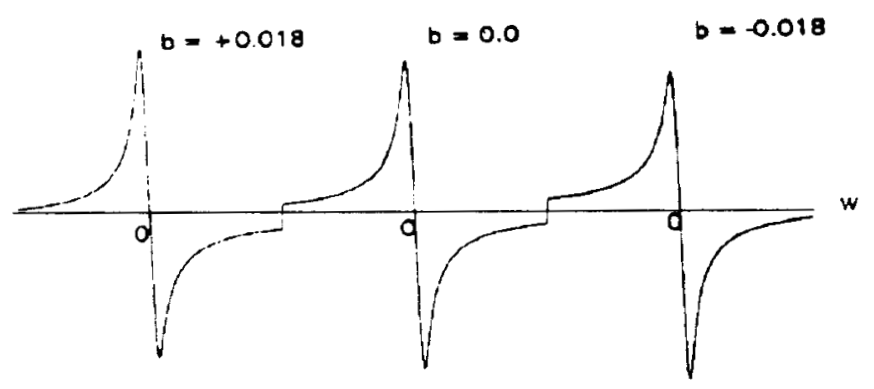

Figura IV-1-B.- Formas de línea de dispersión representadas por la expresión (IV-22) para $a=6$ y $b=0, \pm 0.018$.

Debe mencionarse que es posible agregar nuevos términos a las expresiones (IV-21) y (IV-22) de acuerdo con las reglas mencionadas con anterioridad en este capítulo. Sin embargo, los siguientes términos serán cada vez más complicados y lo más recomendable es acudir a la forma exacta de la FDP y a su forma asimetrizada; ésto será el tema del capitulo $\mathrm{VI}$ en el que se propondrá un procedimiento para asimetrizar la FDP, similar a la empleada en este capítulo. 
Se observa en las expresiones (IV-21) y (IV-22) que para $b=0$ (el perfil simétrico), se recobran las formas simétricas (III-77) y (III-78) como es de esperarse. Es necesario enfatizar que las expresiones (IV-21) y (IV-22) pueden ser usadas para ajustar formas de linea experimentales asimétricas con pequeño contenido gaussiano. También debe señalarse que las expresiones (IV-21) y (IV-22), junto con las condiciones (IV-23), configuran una relación de dispersión porque una es la transformada de Hibert de la otra y cumplen con las condiciones de simetría requeridas.

En la siguiente sección se dará un ejemplo de aplicación a un caso experimental, para valorar la utilidad de los nuevos perfiles de absorción y dispersión asimétricos propuestos en las expresiones (IV-21) y (IV-22).

\section{4.- EJEMPLO DE APLICACION A UN CASO EXPERIMENTAL PARA PROBAR EL MODELO ASIMETRICO DE ABSORCION PROPUESTO}

En esta sección el modelo asimétrico para la línea de absorción representado por la expresión (IV-21) se aplicará al caso experimental de una línea espectral emitida por átomos neutros excitados en un plasma de helio, producido por resonancia ciclotrónica de electrones (RCE). El problema consiste en determinar la temperatura de la componente neutra del plasma. Para ésto se contó con los datos experimentales obtenidos de un experimento cuyos principales parámetros se describirán más adelante [43]. El plasma fué producido en un dispositivo diseñado originalmente para el depósito de películas delgadas de diamante. El dispositivo se describe en la referencia [43], sin embargo es conveniente mencionar aqui que una de sus características es que la magnitud del campo magnético estático externo equivale a la mitad de la magnitud del campo para resonancia a una frecuencia de microondas de $2.45 \mathrm{GHz}$. Bajo esta condición se produce un plasma "sobre-denso"; esto es, la densidad de electrones en 
el plasma es más alta que la densidad critica a $2.45 \mathrm{GHz}$. La "densidad crítica" para este caso se obtiene de la ecuación para la frecuencia de plasma cuando ésta se iguala a $2 \pi$ veces la frecuencia de la microonda:

$$
\omega_{p}=\left(\frac{4 \pi n e^{2}}{m}\right)^{1 / 2}=1.5+x 10^{10} \text { rads.seg } \operatorname{seg}^{-1}
$$

donde $n, e, m$ son la densidad de electrones, la carga y la masa del electrón, respectivamente.

En un régimen de plasma "sobre-denso", se pueden producir inhomogeneidades en la densidad de electrones; debidas a interacciones no lineales entre el campo de microondas y el plasma. Esas inhomogeneidades pueden a su vez aumentar el ensanchamiento Stark de las lineas espectrales emitidas por el plasma. Aparentemente este efecto se encuentra presente en este experimento como se verá a partir de los resultados del presente análisis.

\section{5.- DESCRIPCION DEL PROBLEMA}

El experimento fué realizado por Camps et al. [43] sobre el depósito de películas delgadas y nitruración por medio de un plasma producido por microondas. A partir de estos experimentos, el presente autor dispuso del registro de una de las líneas emitidas por la descarga para estimar la temperatura de la componente neutra del plasma por medio del empleo del modelo de línea presentado en este capitulo.

\section{1. - Los parámetros de la descarga}

El plasma se produce por medio de una descarga generada por microondas sobre helio en un dispositivo de la Gerencia de Ciencias Básicas del ININ que se usa para el depósito de películas delgadas. Los datos de la descarga se listan enseguida. 
Presión de helio: 0.5 mTorr

Frecuencia de la microonda: $2.45 \mathrm{GHz}$

Potencia de la microonda: $200 \mathrm{~W}$

Grado de ionización: 10\%

Campo magnético externo (axil) en el resonador: $437.5 \mathrm{G}$

Campo magnético en la región de observación: $200 \mathrm{G}$

Densidad crítica de electrones: $7 \times 10^{10} \mathrm{~cm}^{-3}$

Densidad de electrones: $+x / 0^{11} \mathrm{~cm}-3$

Temperatura de electrones: $6 \mathrm{eV}$

Los datos correspondientes a la temperatura y densidad de electrones fueron obtenidos por los autores de la referencia [43] por medio de una sonda electrostática simple. Como puede verse, la densidad de electrones que se midió es mayor que la densidad crítica a la frecuencia de la microonda lo cual puede producir fenómenos no lineales en el plasma. Esto último podría afectar los perfiles de las líneas espectroscópicas emitidas por el plasma.

\section{2.- El instrumento de observación}

Los autores de la referencia [43] reportan que el espectro producido por el plasma de helio fué registrado por un espectroscopio de emisión óptica (OES) que consiste de un monocromador Spectra $\operatorname{Pro}^{T M} 275$ y un tubo fotomultiplicador PD-439, ambos controlados por un detector de interfase PS-455, todos ellos de Acton Research Corp. El monocromador recibe la luz recogida por lentes de cuarzo colocadas frente a sendas ventanas de cuarzo en la cámara del plasma. El monocromador tiene una longitud focal de $27.5 \mathrm{~cm}$ con una rendija de entrada y salida de $10 \mu \mathrm{m}$ ancho y una rejilla de 1200 líneas $\mathrm{mm}^{-1}$, todo lo cual produce una resolución de $1 \stackrel{\circ}{A}$ en las mediciones de la emisión del plasma. La señal estudiada se recogió de una zona del plasma situada a lo largo del diámetro de la región de bajo campo magnético; por lo tanto la temperatura 
atómica estimada es el promedio a lo largo del diámetro.

\section{3.- Análisis del espectro registrado}

Para probar lo adecuado del modelo asimétrico de línea propuesto en este capítulo, se eligió la línea $\lambda_{0}=3888.648^{\circ} \mathrm{A}$ del $\mathrm{Hel}$ [44]. Esta línea es un triplete que se origina de la transición $3 p{ }^{3} p \rightarrow 2 s{ }^{3} S$ [45]. Usando información experimental previa [45] asociada con la posición de los términos del triplete, se encuentra que la separación entre ellos es muy pequeña (del orden de $10^{-2} \mathrm{~A}$ ). Además, para un valor de $200 \mathrm{G}$ del campo magnético en la región de observación y considerando la línea $\lambda_{0}=3888.648^{\circ} \AA$, la estimación de la magnitud de la partición Zeeman de la línea (ver por ejemplo las referencias [46] y [47]) es también muy pequeña, del orden de $10^{-3} \stackrel{\circ}{A}$. Debido a que la resolución del instrumento de observación $1 \stackrel{\circ}{A}$ usado en este experimento impide distinguir totalmente la estructura fina de este triplete, se consideró como singulete la línea emitida en esta transición.

El análisis de esta línea, su deconvolución y ajuste se efectuaron usando el modelo de línea asimétrica dado por la expresión (IV-21) y usando el programa MINUIT [48]. Este programa necesitó tres líneas del modelo (IV-21) para lograr el mejor ajuste a la línea experimental elegida. Ver Tabla IV-1.

TABLA I

\begin{tabular}{|l|l|l|l|}
\hline & \multicolumn{1}{|c|}{ Lnea 1 } & \multicolumn{1}{c|}{ Unea 2 } & \multicolumn{1}{c|}{ Unez 3 } \\
\hline Postcton $(\AA)$ & 3383.6 & 3887.4 & 3888.4 \\
\hline Altura & 0.02 & 0.3 & 1 \\
\hline$\Gamma_{L}(\AA)$ & 6.4893 & 2.2595 & $7.3909 \times 10^{-1}$ \\
\hline$\Gamma_{C}(\AA)$ & $3.1119 \times 10^{-2}$ & $1.5251 \times 10^{-2}$ & $2.8623 \times 10^{-2}$ \\
\hline$T(K)$ & 1502.92 & 605.95 & 422.78 \\
\hline$a$ & 173.61 & 123.35 & 21.498 \\
\hline$b$ & $-2.7358 \times 10^{-3}$ & $6.5483 \times 10^{-4}$ & $-5.1049 \times 10^{-3}$ \\
\hline
\end{tabular}

Tabla IV-1.- Parámetros de línea obtenidos después de ajustar la línea de la Fig. IV-2 con tres líneas del tipo (IV-21). La temperatura se calculó por medio de la expresión (IV-24). 
Al analizar la línea experimental elegida se obtuvo un perfil de estructura compleja centrado en el triplete de helio elegido. En la Figura IV-2 se muestran los puntos experimentales (representados por cruces) del perfil registrado; también se muestra, en línea continua, la curva que mejor se ajusta a los puntos experimentales usando el modelo de línea representado por la expresión (IV-21). Un breve examen de los puntos experimentales de la línea muestra un pico bien resuelto (que se denominará Línea 3), centrado alrededor del triplete de helio elegido; se observa también un hombro bien marcado (que se denominará Línea 2) localizado del lado de las bajas longitudes de onda. El procedimiento de ajuste detecta también una línea adicional de amplitud muy pequeña (que se denominará Línea 1) localizada a la izquierda del hombro. Esta línea no se observa a simple vista. En efecto, el mejor ajuste al perfil registrado se obtuvo con el empleo de tres lineas del tipo (IV-21).

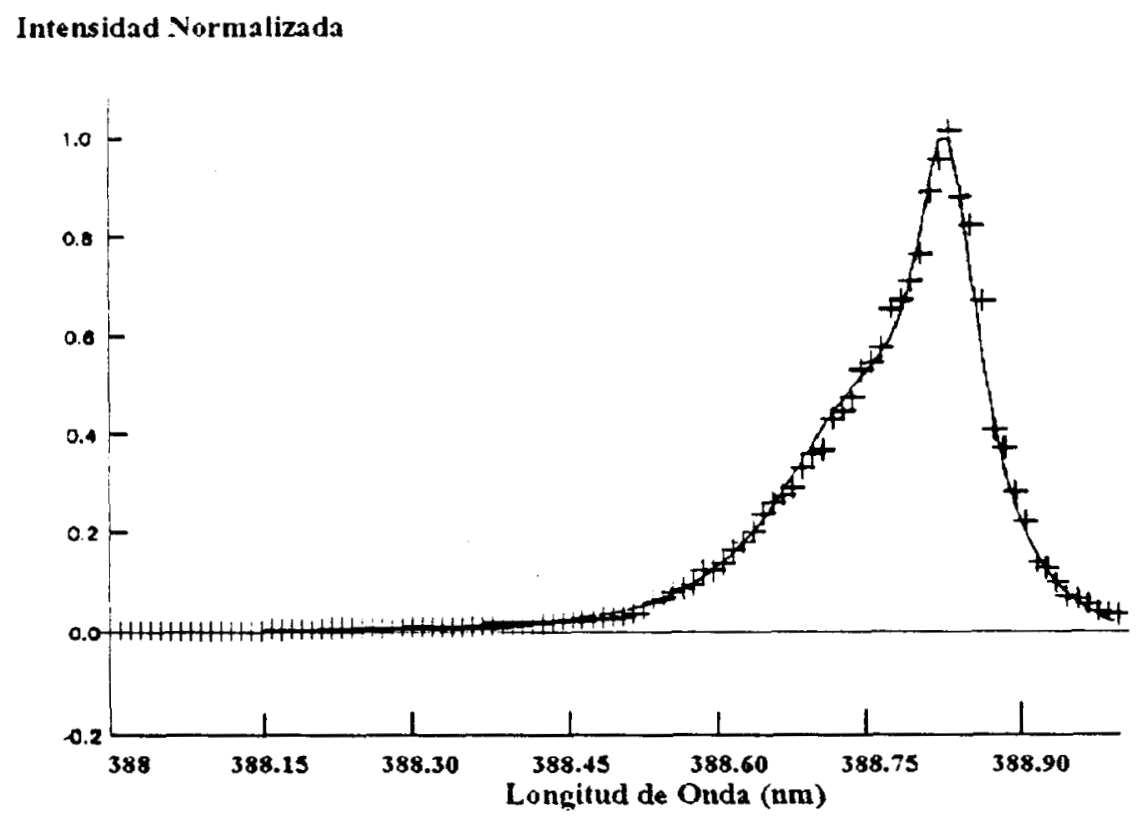

Figura IV-2.- Ajuste de los puntos experimentales $(+)$ alrededor de la línea $\lambda=3888.648 \stackrel{\circ}{A}$ emitida por helio excitado. El ajuste se realizó por medio de la superposición de tres líneas del tipo (IV-21). Los parámetros de línea se reportan en la Tabla I. 
El procedimiento de ajuste y deconvolución proporciona las posiciones, las amplitudes, las anchuras $\Gamma_{L}$ y $\Gamma_{G}$ para cada línea y los parámetros $a$ y $b$. Estos valores se reportan en la Tabla IV-1. Los coeficientes de correlación para la estimación de esos parámetros se reportan en la Tabla IV-2.

TABLA II

\begin{tabular}{||c|c|c|c|}
\hline \multicolumn{4}{|c|}{ COEFICIENTES DE CORRELACION } \\
\hline & Linea 1 & Linea 2 & Linea 3 \\
\hline$\Gamma_{\mathrm{L}}$ & 0.98480 & 0.89021 & 0.98222 \\
\hline$\Gamma_{\mathrm{C}}$ & 0.76024 & 0.65294 & 0.96838 \\
\hline
\end{tabular}

Tabla IV-2.- Coeficientes de correlación obtenidos durante la estimación de los anchos gaussianos de las tres líneas.

\section{4.- Cálculo de las temperaturas}

Para el cálculo de la temperatura es necesaria una fórmula que la relacione con uno de los parámetros mencionados. Es bien conocido el efecto de ensanchamiento que tiene el movimiento aleatorio de los átomos emisores sobre las lineas espectrales en plasmas tenues [46], [47]. Al ser caótico dicho movimiento, el ensanchamiento producido es de tipo gaussiano y la anchura de la línea, a la mitad del máximo, puede escribirse como (Ref. [46], pág. 269):

$$
\Gamma_{G}=7.16 \times 10^{-7} \lambda_{0}(T / M)^{1 / 2} .{ }^{\circ} .
$$

donde $T$ está expresada en Kelvins y $M$ en unidades de masa atómica; $\lambda_{0}$ es la longitud de onda para cada línea. Después de realizar los cálculos numéricos pertinentes con el objeto de calcular la temperatura para cada línea, considerando las masas de los distintos emisores y los anchos gaussianos correspondientes, los valores obtenidos se reportan en la Tabla IV-1. 


\section{5.- Análisis de los resultados}

En esta sección se discutirán varios aspectos de la información contenida en la Tabla IV-1.

1.- La línea calculada, $\lambda=3888.4 \stackrel{\circ}{\AA}$, está corrida hacia la izquierda en $0.248 \stackrel{\circ}{A}$ con respecto a la línea teórica del helio excitado, $\lambda=3.888 .648 \stackrel{\circ}{\AA}$ [44], [45]. Análogamente, la línea calculada, $\lambda=3883.6^{\circ} \mathrm{A}$, está corrida hacia la izquierda en $0.224^{\circ} \mathrm{A}$ con respecto a la línea teórica de carbono ionizado, $\lambda=3883.82+{ }^{\circ} 4$ [44], [45]. En contraste, la línea calculada, $\lambda=3887 .+\stackrel{\circ}{A}$, está corrida hacia la derecha en $0.266^{\circ} \mathrm{A}$ con respecto a la línea teórica del neón excitado, $\lambda=3887.134 \stackrel{\circ}{A}$ [44], [45]. En los primeros dos casos el corrimiento podría ser explicado en términos de la falta de buena calibración del aparato. Sin embargo, no se propone alguna explicación para el corrimiento que ocurre en el tercer caso. A pesar de lo anterior, esos corrimientos son irrelevantes para el propósito de la determinación de las temperaturas a través del ancho gaussiano de las líneas espectrales.

2.- Los valores relativamente grandes obtenidos para la anchura lorentziana $\Gamma_{L}$ pueden ser debidos a los efectos que sobre la densidad de electrones tiene la interacción no lineal entre el campo de microondas y el plasma "sobre-denso". En efecto, la magnitud de las anchuras naturales lorentzianas es de aproximadamente $10^{-4} \stackrel{\circ}{A}$. Si bien se puede esperar una anchura lorentziana grande en este tipo de experimentos, los valores obtenidos por medio del presente ajuste son muy grandes, particularmente los de las Líneas 1 y 2 (aquellas que tienen las menores intensidades; $c f$. la Tabla $\mid V-1$ ). La Línea 3 presenta una anchura lorentziana que es más 
realista aunque todavia es grande comparada con aquellas esperadas a mayores temperaturas y densidades electrónicas; ver por ejemplo los datos para He II en la Ref. [46] pág. 267.

3.- Los valores obtenidos para las anchuras gaussianas de las tres líneas son del mismo orden de magnitud. Esto significa que las tres líneas estan respondiendo de manera similar a los movimientos térmicos de sus emisores. Por otra parte, en vista de que la partición Zeeman es aproximadamente un orden de magnitud menor que los valores de las anchuras gaussianas $\Gamma_{G}$ presentadas en la Tabla IV-1, debe hacerse una pequeña corrección en las temperaturas calculadas de la Tabla IV-1, si se desea obtener un valor más preciso de la temperatura.

4.- Los valores de la temperatura de la Tabla IV-1 fueron obtenidos con ayuda de la fórmula (IV-24). Se desea proponer ahora que el criterio para elegir el valor de la temperatura que caracterice a la componente neutra del plasma en el presente experimento, se base en el más alto valor del coeficiente de correlación para la estimación de las anchuras $\left(\Gamma_{L}, \Gamma_{G}\right)$. Este criterio, es satisfecho por la Línea 3, de acuerdo con la Tabla IV-2. Por lo tanto, el valor $T=422.78 \mathrm{~K}$ es la estimación para la temperatura de los átomos neutros excitados en el plasma del presente ejemplo. El valor $T=422.78 \mathrm{~K}$ representa una estimación que está en el intervalo de valores reportado en la literatura para la temperatura de átomos neutros excitados en descargas similares con parámetros similares [49]. Esto significa que el modelo de línea asimétrico (IV-21) propuesto en este trabajo, puede ser de utilidad para la deconvolución y el ajuste de líneas experimentales a fin de obtener información importante acerca de los parámetros espectroscópicos de un plasma. Es necesario mencionar que también se realizó el ajuste usando el perfil simétrico (III-77) (caso $b=0$ ), 
obteniéndose valores más pequeños para los coeficientes de correlación. Esto significa que las líneas que componen la línea experimental elegida poseen cierto grado de asimetría.

5.- Con respecto al parámetro de asimetria estimado para las líneas de la Tabla IV-1, se nota que sus valores son relativamente pequeños indicando que las líneas son prácticamente șimétricas. Por otra parte, los valores de $b$ reportados en la Tabla IV-1 están dentro del intervalo $|b| \leq 0.08$.

6.- Análogamente, los valores estimados del parámetro a reportados en la Tabla IV-1, satisfacen la condición $a \gg \frac{1}{\sqrt{2}}$. En esta forma los valores obtenidos de $a$ y $b$ son consistentes con los intervalos de aplicabilidad impuestos sobre las expresiones (IV-21) y (IV-22).

Como puede verse de la Tabla IV-1, las separaciones entre las tres líneas encontradas con el ajuste, son mucho mayores que las separaciones entre los términos individuales de la línea de helio y que el tamaño de su partición Zeeman. Esto significa que las Lineas 1 y 2 de la Tabla IV-1 no son miembros del triplete $3888.648^{\circ} \mathrm{A}$. La débil Línea 1 podría estar asociada con la línea $\lambda=3883.824 \stackrel{\circ}{A}$ emitida por la impureza de carbono ionizado $C$ Il en la transición $3 d^{4} F \rightarrow+f{ }^{4} G$ [44], [45], significando ésto que una pequeña fracción de carbón pudiera estar presente en la cámara del plasma. La temperatura calculada asociada con esta línea y que se reporta en la Tabla IV-1, es de $1502.92 \mathrm{~K}$. Esta temperatura tan elevada podría ser el resultado de un efecto de calentamiento debido a la aceleración de los iones de carbono en el campo de la microonda. En efecto, los iones y los electrones tienen tipicamente energías en el intervalo 0.1-20 eV en plasmas generados por resonancia ciclotrónica de electrones [49]. Por otra parte, la pequeña Línea 2 podria estar asociada con la línea $\lambda=3887.134 \stackrel{\circ}{A}$ emitida por Ne I en la transición 3cl [ $11 / 2] \rightarrow 3 s$ [1/2.] [44], [45], lo cual indica la posible presencia de pequeñas cantidades de neón en la descarga. La temperatura calculada asociada con esta línea y que se reporta en la Tabla IV-1, es 
de $605.95 \mathrm{~K}$. El calentamiento no muy grande de la población de neón podría deberse, por una parte, a que los iones de carbono no transfieren momento a los de neón en forma eficiente porque su masa es más pequeña que la de los de neón y por otra parte porque la población de átomos de carbón es muy pequeña.

En este capítulo, se ha mostrado cómo se recobran los términos del desarrollo asintótico de la función de dispersión del plasma partiendo de una forma aproximada del perfil de Voigt reportada en la literatura y uno de cuyos autores es el presente. Lo anterior se hizo tomando ventaja de la relación que existe entre el perfil de Voigt y la parte imaginaria de la FDP por un lado y por el otro, de la relación entre el perfil de dispersión y la parte real de la FDP. Ambas relaciones están conectadas por la transformada de Hilbert.

El procedimiento usado aquí para obtener la serie asintótica de la FDP sugiere el uso de ésta como un medio alternativo para el análisis de la estructura de líneas espectrales voigtianas. Esto puede ser de relevancia para el espectroscopista que desea hacer determinaciones de anchuras de líneas para evaluar por ejemplo la magnitud de efectos de relajamiento y los debidos a mecanismos estocásticos tales como temperatura y resolución instrumental, que pueden modificar la forma de los perfiles de línea.

Además, como muchas líneas espectrales tienen algún grado de asimetría, se realizó una generalización de los perfiles simétricos aproximados de absorción y dispersión, que consiste en incluir un parámetro de asimetría. Debido a que los nuevos perfiles están conectados entre ellos por la transformada de Hilbert, forman una relación de dispersión limitada por las condiciones $a \gg \frac{1}{\sqrt{2}}$ y $b \leq 0.08$.

Hay un aspecto adicional que es necesario mencionar aqui y es el siguiente: De acuerdo con lo visto en este capítulo los perfiles (III-77) y (III-78) pueden ser extraídos del desarrollo asintótico de la FDP. Como se ha dicho, ésta tiene incorporada información física a través de sus contenidos lorentziano y gaussiano. Además, las partes real e imaginaria se pueden usar para representar los perfiles espectrales de 
dispersión y absorción, respectivamente, en muchas espectroscopías, pero con mayores posibilidades ahora que ha sido incorporado un parámetro de asimetria. Por lo tanto, el ajuste de estos perfiles a líneas experimentales puede proporcionar información física relevante acerca del sistema analizado, como en el caso del ejemplo reportado aquí, a diferencia de perfiles como los de la referencia [42] que están caracterizados por parámetros sin significado físico.

En el siguiente capitulo se seguirá explotando el par simétrico absorción-dispersión para el caso de una pequeña contribución gaussiana $\left(a \gg \frac{1}{\sqrt{2}}\right)$, compuesto por las expresiones (IV-77) y (IV-78). Alli se hará uso del concepto DISPA (DISPersión-Absorción) y además se propondrá un nuevo método, no basado en técnicas de ajuste, para analizar líneas voigtianas con pequeña contribución gaussiana empleando datos experimentales de absorción y dispersión (DISPA). El método será aplicado a una línea experimental de RPE previamente analizada en la literatura por nosotros y otros autores (cf. Ref. [26]). 


\section{CAPITULO V}

\section{UN NUEVO METODO SIMPLE PARA ANALIZAR VOIGTIANAS DE PEQUEÑO CONTENIDO GAUSSIANO CON AYUDA DE DATOS DISPA}

\section{INTRODUCCION}

En este capitulo se avanzará más en el estudio del par simétrico absorción-dispersión constituido por las expresiones (III-76) y (III-78) con el objeto de continuar explotándolo y generar con él información adicional a la que se obtuvo en el Capitulo IV. En efecto, aqui se propondrá un nuevo método, caracterizado por su sencillez, para estudiar formas de linea voigtianas con bajo contenido gaussiano $\left(a \gg \frac{1}{\sqrt{2}}\right)$ con la ayuda de datos espectrales DISPA (DISPersión-Absorción). Debe hacerse énfasis en que el método aqui propuesto no está basado en técnicas numéricas de ajuste. El método sirve para analizar líneas simétricas aisladas no traslapadas en cualquier espectroscopia ya que se manejan tanto el perfil de absorción como el de dispersión [8].

Para el estudio de muestras de substancias, en la mayor parte de las espectroscopias (óptica, EPR, Mössbauer, etc) se usa únicamente el perfil de absorción. debido a su mejor resolución y simetría. Sin embargo, cuando se desea emplear la técnica DISPA, es necesario disponer tambien del perfil de dispersión. En espectroscopia de radiofrecuencia (Resonancia Magnética Nuclear, Resonancia Ciclotrónica de lones, Resonancia Cuadrupolar Nuclear) y de microondas (RPE, Resonancia Puramente Rotacional) es posible obtener los espectros de absorción y de dispersión con los cuales se pueden elaborar gráficas DISPA. En efecto, hace varios años Talpe y Van Gerven [50] consideraron que la dispersión en resonancia constituye también una herramienta útil para la investigación de propiedades magnéticas de substancias. Posteriormente, Marshall y Roe [51, 52] construyeron, por primera vez, 
diagramas DISPersión vs. Absorción (DISPA) de líneas espectrales para estudiar ensanchamiento de líneas en varias situaciones. Los diagramas DISPA consisten en graficar las expresiones (o los datos experimentales) para los perfiles de dispersión y absorción, normalizados a la altura máxima de la absorción, en el eje vertical y horizontal, respectivamente. Esto se verá en la siguiente sección. El diagrama DISPA de una línea lorentziana pura es un círculo perfecto de radio unitario [51, 53]. Cualquier desviación de este círculo de referencia en un diagrama DISPA proporcionainformación acerca de la clase de mecanismo ensanchador que acompaña a la transición [53]. Más recientemente Miglierini y Sitek [54] y Miglierini [55] aplicaron el método DISPA para estudiar líneas Mössbauer; en tanto que Craig y Marshall [56] construyeron diagramas DISPA de varias líneas para hacer correcciones de fase en espectros de Resonancia Magnética Nuclear con Transformada de Fourier.

De lo anterior se infiere que existe interés en el uso del método DISPA para obtener información importante de líneas espectrales. Por ello, en en este capítulo, se propondrá un nuevo método para estudiar perfiles de línea con ayuda de datos DISPA, el cual puede contribuir a ampliar el conocimiento sobre el tema del análisis de estructura de líneas espectrales en varias espectroscopias.

Como un ejemplo de aplicación del método, se calcularán las contribuciones lorentziana y gaussiana en la línea RPE de una solución de potasio en amonio, reportada por Hutchison y Alger $[57,58]$. El cálculo se realizará por medio de una operación de deconvolución que no se basa en el ajuste numérico de datos. La elección de esta línea se debe a que posee una pequeña contribución gaussiana $[57,58]$ por lo que puede ser estudiada con el método aquí presentado, que es válido para el caso de $a \gg \frac{1}{\sqrt{2}}$. Además, el hecho de tener una forma bien definida $v$ un grado muy pequeño de asimetría la convierte en un buen sujeto para probar el presente método.

El par absorción-dispersión, para el caso de pequeña contribución gaussiana comparada con la contribución lorentziana $\left(\imath \gg \frac{1}{\sqrt{2}}\right)$ [33], está constituido por las 
expresiones (III-76) y (III-78) que, como se mencionó en el capítulo anterior, forman una relación de dispersión. El procedimiento se basa en el analisis del perfil de dispersión para determinar sus extremos y en el comportamiento de éstos en el diagrama DISPA.

La operación de deconvolución de la línea RPE de potasio en amonio se realiza obteniendo primero una lista de datos DISPA para esta línea; enseguida estos datos se usan en combinación con los resultados del análisis de la expresión para el perfil aproximado de dispersión a fin de completar el cálculo. Los valores resultantes de los anchos gaussiano y lorentziano se comparan entonces con los obtenidos por Jiménez-Domínguez et al. [26]. Estos autores realizan la deconvolución ajustando los datos de la línea con las expresiones aproximada y exacta del perfil de Voigt. La comparación es buena y se concluye que el método que se propone en este capítulo, puede ser aplicado fácilmente a líneas en cualquier espectroscopía que puedan ser representadas por voigtianas que tengan pequeña componente gaussiana y un grado muy pequeño de asimetría.

\section{2.- FUNDAMENTOS DEL METODO}

El perfil de absorción voigtiano exacto está representado por la expresión (II-6) mientras que el voigtiano aproximado, por la expresión (III-76). En estas expresiones la variable adimensional " está dada por (II-4a) y el parámetro a por (II-4b). La variable física $\vee$ mide la posición espectral $y \quad v_{0}$ es el valor donde ocurre la resonancia. En el caso de las espectroscopias de RPE y de RMN, $\vee$ representa el valor del campo magnético y de la frecuencia, respectivamente. Las expresiones para $v$ y a son muy importantes para el uso del método propuesto en este capítulo puesto que serán usadas para deducir los valores de $\Gamma_{L} \vee \Gamma_{G}$. En el ejemplo que se propondrá, $\vee$ se conoce del experimento ya que el perfil de la línea se registra conforme el espectrómetro barre el campo magnético. Por otra parte, el perfil aproximado de dispersión está dado por (III-78). Nótese que (III-76) y (III-78), al depender de $v$ y $a$, sirven para generar datos DISPA que servirán para construir gráficas DISPA, como se indicará después, y ajustar 
espectros de absorción y de dispersión. Ahora bien, si se desea trazar una gráfica DISPA con las expresiones aproximadas (III-76) y (III-78), deben considerarse las expresiones normalizadas al máximo de la absorción, para la DISPersión (la ordenada) y para la Absorción (la abscisa) de la siguiente manera

$$
D_{N}(v, a)=\frac{D_{A P R}(v, a)}{H_{A P R}(0, a)} \quad(v-1 a)
$$

v

$$
H_{N}(u, a)=\frac{H_{A P R}(u, a)}{H_{A P R}(0, a)}
$$

Adviértase de la expresión (III-78) que los intervalos $-\infty<v<0$ y $0<v<\infty$ reproducirán las partes superior e inferior de la gráfica DISPA, respectivamente. Por otro lado, se puede deducir de la expresiones ( $V-1)$ que en el límite $a \rightarrow \infty$, (caso puramente lorentziano para cualquier valor de $\Gamma_{L}$ l, la gráfica DISPA tiende a un círculo centrado en $\left(H_{N}=\frac{1}{2}, D_{N}=0\right)$, como es de esperarse.

Una gráfica DISPA puede ser caracterizada por los puntos $U_{\max }$ en los cuales la dispersión (III-78) tiene extremos. La localización de los extremos se realiza de la siguiente manera: Tomando la derivada de (III-78) con respecto a la variable $v$, se obtiene una ecuación cúbica en la variable $u$, cuyas soluciones reales y positivas producen los puntos $v_{\max }$. en los cuales se presentan los extremos para diferentes valores de $a$. La ecuación cúbica resultante es

$$
2\left(a^{2}-u\right)\left(a^{2}+u\right)^{2}-3\left(a^{4}+u^{2}-6 a^{2} u\right)=0
$$

donde $u=v_{\max }^{2}$.

A manera de ejemplo la Tabla $V-1$ muestra los valores de $v_{\max }$, calculados por medio de la expresión ( $V-2)$, para algunos valores de $a>\frac{1}{2}$. 


\begin{tabular}{|c|c|}
\hline$a$ & $\pm v_{\max }$ \\
\hline 4.0 & 4.1826 \\
\hline 4.5 & 4.6633 \\
\hline 5.0 & 5.1476 \\
\hline 5.5 & 5.6346 \\
\hline 6.0 & 6.1236 \\
\hline 6.5 & 6.6143 \\
\hline 7.0 & 7.1063 \\
\hline 8.0 & 8.0932 \\
\hline 9.0 & 9.0829 \\
\hline 10.0 & 10.0747 \\
\hline
\end{tabular}

TABLA V-1.- Esta tabla muestra algunos valores de $v_{\max }$ que se obtienen como soluciones, cuando diferentes valores de $a>\frac{1}{\sqrt{2}}$ se substituyen en la ecuación (V-2). Esta tabla es válida sólo cuando los perfiles de absorción y dispersión son los representados por las expresiones (III-77) y (III-78), respectivamente.

Se puede notar de esta tabla que los extremos de la gráfica DISPA ocurren siempre en valores de $a$ tales que $\left|u_{\max }\right| \geq a$. Entre más alto sea el valor de $a$, mejor es el parecido de $v_{\max }$ al valor de $a$. En efecto, los extremos de la dispersión para una lorentziana pura $(a \rightarrow \infty)$ de anchura $\Gamma_{L}$ se presentan en $v_{\max }= \pm c$ como puede verificarse a partir de la expresión (III-78). La relación $v_{\max }= \pm c$ puede ser escrita en términos de la variable física de campo magnético $v$ como $v_{\max }= \pm \frac{\Gamma_{L}}{2}$, donde en este caso $\Gamma_{L}$ tiene unidades de campo magnético.

Los puntos para los cuales $D_{A P R}(U, a)$ tiene sus extremos, corresponden aproximadamente a los puntos en los que el perfil de absorción alcanza la mitad de su altura máxima. Esto significa que si cualquier par de valores $\left(u_{\max }, a\right)$ de la Tabla $V$-1 se introduce en ( $V .-1 b$ ) (la absorción normalizada), se obtiene un valor ligeramente mayor que 0.5. Este valor tiende a 0.5 en el límite $a \rightarrow \infty$. 
A continuación se analizará grosso modo el comportamiento del máximo de la gráfica DISPA correspondiente a las expresiones ( $V-1)$. Para este fin se elegirá la parte superior de la gráfica para la cual el máximo se localiza cerca del punto $u_{\max } \cong-\alpha$. Entonces, desarrollando en serie estas expresiones alrededor de a les decir, alrededor de un punto cercano al máximo) y conservando términos hasta cuarto orden en el parámetro $a$, la forma aproximada de estas en el máximo es

$$
\begin{aligned}
& H_{N} \cong \frac{1}{2}\left[1+\frac{3}{4 a^{2}}+\frac{3}{8 a^{4}}\right] . \\
& D_{N} \cong \frac{1}{2}\left[1 \cdot+\frac{1}{4 a^{2}}+\frac{1}{8 a^{4}}\right] .
\end{aligned}
$$

El análisis cuidadoso de las expresiones (V-3), que son válidas para $a \gg \frac{1}{\sqrt{2}}$,

permite hacer las siguientes observaciones. Para un espectro del tipo voigtiano que tiene mayor contenido lorentziano que gaussiano (presente caso), el máximo de la gráfica DISPA (en la parte superior de la gráfica) está corrido hacia arriba y hacia la derecha con respecto al de la gráfica DISPA para un par absorción-dispersiónpuramente lorentziano. Un corrimiento en el sentido opuesto será denominado como "anómalo", en este capítulo. Más adelante en este mismo capítulo se mostrarán gráficas DISPA para un caso particular. De las expresiones $(V-1)$ se puede ver que $H_{N}=D_{N}=\frac{1}{2}$ en el máximo conforme $a \rightarrow \infty$, para una lorentziana pura. El correspondiente análisis en la parte inferior de la gráfica DISPA muestra que el corrimiento se produce hacia abajo y hacia la derecha con respecto al máximo de la gráfica para una lorentziana pura. Esto último es una consecuencia de las propiedades de simetría de (III-77) y (III-78). Es preciso notar que las expresiones ( $V-3$ ) no pueden ser usadas para hacer un análisis similar en el caso de voigtianas cuyo contenido gaussiano es mayor que el lorentziano (caso de a pequeña), puesto que dichas expresiones sólo son válidas cuando se cumple la condición $a \gg \frac{1}{\sqrt{2}}(a$ grande) . 
Al aplicar el presente método, los valores de los parámetros $\Gamma_{L}$ y $\Gamma_{G}$, para una línea con $a \gg \frac{1}{\sqrt{2}}$ pueden ser encontrados usando las expresiones (II-4a), (II-4b), (V-2) y los datos de dispersión de la linea.

\section{3.- APLICACION DEL METODO}

\section{1.- APLICACION A UNA LINEA HIPOTETICA}

Con el objeto de mostrar con claridad el uso del presente método $y$ antes de aplicarlo a una línea experimental, se considerará primero una línea RPE hipotética la cual es registrada, como usualmente se hace, como la primera derivada del perfil de absorción. Esta línea hipotética tendrá además un perfil bien definido y con una asimetría muy pequeña. En primer lugar es necesario obtener los datos DISPA de la línea. Para ésto, debe obtenerse una lista de alturas de la línea vs campo magnético (o frecuencia); esto se hace a través de un proceso de digitización sobre el perfil que representa a la primera derivada de la absorción. Este proceso permite obtener $N$ puntos-dato igualmente espaciados que corresponden a un cierto intervalo de campo (o de frecuencia) $[-\vee, v]$ asociado con el campo magnético aplicado. En vista de que la posición de la línea no es de interés para los propósitos del presente método, se supondrá que la linea está centrada en $v_{0}=0$. Puesto que la línea se registra en el espectrómetro como la primera derivada del perfil de absorción, es necesario tomar la anti-derivada de los datos espectrales digitizados igualmente espaciados, para obtener el perfil de absorción. Este paso se omite en espectroscopías en las que se registra directamente el patrón de absorción en lugar de su derivada.

Enseguida se toma numéricamente la transformada de Hilbert de los datos de absorción para obtener el perfil de dispersión. Esto se hace usando la siguiente receta 
(Ref. [17], pp 17, 109) cuya base formal está contenida en el material presentado en la sección 5.2 del capítulo III, sobre el uso de la función signum para derivar las relaciones de Kramers-Krönig:

1).- Tómese la transformada inversa rápida de Fourier (IFFT) de los datos integrados para pasar al dominio del "tiempo". Este paso requiere primero que los datos de absorción sean completados con ceros a cada lado del pico hasta obtener $2^{n}$ puntos o canales, como lo requiere el algoritmo de la transformada rápida de Fourier; $n$ es un entero. El resultado de este paso puede representarse simbólicamente por una función compleja de la forma $f(t)=R(t)+i l(t)$.

2).- Multiplíquense las partes real e imaginaria del resultado por la función signum del tiempo sgln ( $($ ), ver sección 5.2 del capítulo III. Este paso puede representarse simbólicamente por una función compleja de la forma $\operatorname{sgn}(t) f(t)=\operatorname{sgn}(t) R(t)+i \operatorname{sgn}(t) l(t)$.

3).- Tómese la transformada rápida de Fourier (FFT) del producto complejo resultante con el objeto de obtener la función de dispersión en el dominio de la "frecuencia" (campo magnético). Usando la expresión (III-51), esta operación puede ser representada por una cantidad compleja proporcional a:

$$
i \frac{1}{\pi} P \int \frac{R\left(\omega^{\prime}\right) d \omega^{\prime}}{\omega-\omega^{\prime}}-\frac{1}{\pi} P \int \frac{l\left(\omega^{\prime}\right) d \omega^{\prime}}{\omega-\omega^{\prime}} .
$$

4).- Finalmente tómese la parte real del resultado.

Del resultado obtenido en el cuarto paso de esta receta puede observarse que se cumple con lo indicado por la expresión (III-65), ya que $I \mathrm{~m}(\omega)$ representa un perfil de absorción cuya transformada de Hilbert produce el correspondiente perfil de dispersión.

De acuerdo con las expresiones ( $\mathrm{V}-1)$, los conjuntos de datos de absorción y dispersión asi obtenidos deben ser normalizados dividiendo ambos conjuntos de datos entre el valor máximo de la absorción. Procediendo en esta forma, se dispone de los 
datos normalizados de absorción y dispersión (datos DISPA). Nótese que con este procedimiento la variable física $v$. la variable voigtiana $v$, $v$ el número de canal $c h$, quedan todos relacionados por una correspondencia biunívoca. Con el objeto de que la variable adimensional $v$ quede centrada en $v=0$, ésta se escribe en términos del número de canal ch como sigue:

$$
u=\frac{\left(c h-2^{n-1}\right)}{f},
$$

donde el factor $f$ ha sido introducido para escalar $v$ con las unidades naturales del papel graduado sobre el cual se llevó a cabo el proceso de digitización. En esta forma los $2^{n}$ valores de la variable $u$, centrados en $u_{0}=0$, quedan colocados en el intervalo adimensional $[-u, v]$ correspondiente al nuevo intervalo de frecuencias $[-v, v$, ] extendido, como se indicó antes, por los ceros agregados a cada lado del pico.

Con la información producida por el procedimiento descrito y suponiendo que el perfil hipotético es del tipo voigtiano con una pequeña contribución gaussiana, el espectroscopista está listo para obtener los valores de $\Gamma_{L}$ y $\Gamma_{G}$ como se indica a continuación:

Se toma cualquier par de valores $(v, v)$, de la lista de datos y substituyéndolo en la expresión (II-4a), se obtiene inmediatamente el valor de $\Gamma_{C}$.

A continuación, se toma de la lista de datos, el valor $v_{\max }$ correspondiente al máximo valor de $D(u, a)$ elegido de acuerdo con el criterio basado en el significado de las expresiones ( $V-3$ ) como se indica en la siguiente sección.

\section{2.- CRITERIO PARA ELEGIR $v_{\max }$ DE LOS DATOS DISPA}

El criterio para elegir $v_{\max }$ a partir de los datos DISPA de una línea espectral experimental debe ser basado en el significado de las expresiones (V-3). 
El máximo de la gráfica DISPA para una línea voigtiana con pequeña contribución gaussiana, está corrido hacia arriba y hacia la derecha con respecto al máximo de la gráfica DISPA para una lorentziana pura. Esto significa que de los datos experimentales normalizados, $v_{\max }$ se determina al considerarse el punto $\left(H_{N}, D_{N}\right)$ que satisfaga las tres condiciones siguientes:

a).- El valor de las coordenadas $H_{N}$ y $D_{N}$, debe ser mayor que 0.5.

b).- El valor de $H_{N}$ debe ser mayor que el valor de $D_{N}$, como se establece por las expresiones ( $V-3)$.

c).- Debe buscarse el mayor de los valores de $D_{N}$ que sea consistente con las condiciones a) y b).

En esta forma, el valor para $u_{\max }$ puede ser localizado en la colección de datos. En seguida el valor de $u=v_{\max }^{2}$ se substituye en la expresión (V-2), cuya solución produce el valor de $a$ que caracteriza la línea. Finalmente, este valor de $a$ y el valor de $\Gamma_{G}$ obtenido con anterioridad se substituyen en la expresión (II-4b) para obtener el valor de $\Gamma_{L}$ para la línea.

El otro valor de $v_{\max }$ puede ser obtenido a partir del mínimo de la gráfica DISPA el cual está colocado en la parte inferior de la misma donde los valores de $D_{N}$ son negativos. Por lo tanto, para encontrar el otro valor de $v_{\max }$, las condiciones sobre las coordenadas $\left(H_{N}, D_{N}\right)$ son las mismas que arriba pero esta vez debe considerarse el valor absoluto $\left|D_{N}\right|$.

El nuevo método propuesto en este capitulo para obtener los valores $\Gamma_{G}$ y $\Gamma_{L}$ de una linea experimental voigtiana, ha sido desarrollado suponiendo que el perfil de la línea experimental bajo estudio no está distorsionado por cualquier otro mecanismo perturbador y que no está tomado en cuenta en el modelo de línea representado por la expresión (III-77). Sin embargo, más adelante se mostrará que, aun en el caso de que la línea esté distorsionada por esos mecanismos, todavia se puede obtener información valiosa usando el presente método. 


\section{3.- APLICACION DEL METODO A UNA LINEA EXPERIMENTAL REAL}

En esta sección se aplicará el método descrito a los datos espectrales experimentales de la línea RPE producida por una solución de potasio en amonio. La elección de esta linea se debe a su perfil bien definido y a su pequeño grado de asimetría. Esta línea fué encontrada en la literatura $[57,58]$ y está reportada como la primera derivada de la absorción. Sin embargo, como se verá de su gráfica DISPA, la línea presenta una distorsión probablemente del tipo de ensanchamiento por modulación [40, p-298]. La Figura V-1 muestra los perfiles de absorción y dispersión obtenidos como se indicó anteriormente. En esta figura, la variable física $\vee$ (NU en la figura), la variable voigtiana $v$ y el número de canal ch se muestran explícitamente sobre los ejes de abscisas, con el objeto de mostrar su correspondencia mutua.

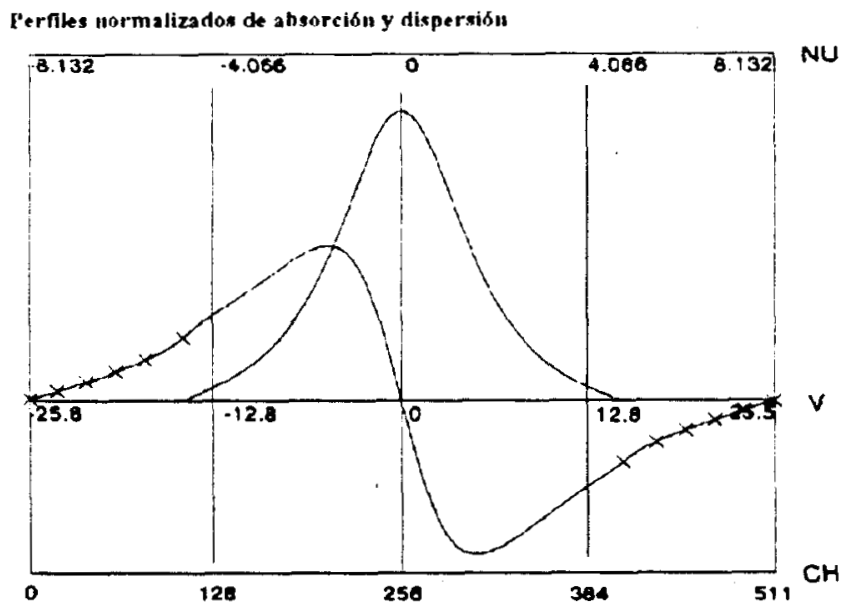

Figura V-1.- Perfiles normalizados de absorción y dispersión de la línea RPE de potasio-amonio. Los perfiles están normalizados de acuerdo con las expresiones ( $\mathrm{V}-1 \mathrm{a}$, b). Las zonas del perfil de dispersión que tienen cruces corresponden a las partes de los datos que se completaron con ceros. Las abscisas representan: el número de canal ch, la variable adimensional $v$ y la variable física $v$, indicada por NU en la figura, en unidades de 0.05 Gauss.

Los datos DISPA de la línea se obtienen conforme a la manera descrita previamente y se hace así: por medio de un proceso de digitización, se extrae un 
conjunto de puntos-dato de la línea que representa la primera derivada del perfil de absorción. El proceso de digitización consiste simplemente en leer las abscisas y las ordenadas de varios puntos igualmente espaciados del perfil. La lectura se puede realizar con facilidad despues de acomodar cuidadosamente sobre la gráfica amplificada de la línea espectral una hoja de papel milimétrico transparente. Entre más puntos se lean es estadísticamente mejor. Por supuesto que es posible realizar más eficientemente esta operación mediante el uso de un "scanner". Puesto que se usó papel milimétrico para esta operación, el factor $f$ de la expresión ( $V-4)$ asume el valor de 10, ya que la unidad natural es $1 \mathrm{~cm}$. En el presente caso se tomaron 311 puntos-dato igualmente espaciados de la variable física $v$, correspondientes al intervalo de campo magnético [-4.924, 4.956], en unidades de 0.05 Gauss. Los 311 puntos-dato se completan con ceros a cada lado del pico para completar $2^{9}=512$ puntos (o canales), como lo requiere el algoritmo de la transformada rápida de Fourier. En esta forma se obtienen 512 valores de la variable $u$, centrados en $v=0 \quad y$ acomodados en el intervalo adimensional $[-25.6,25.5]$, correspondiente al nuevo intervalo de "frecuencias" $[-8.101,8.133]$, en unidades de 0.05 Gauss, extendido por la adición de ceros en ambos lados del pico. El siguiente paso es obtener la antiderivada numérica de esos datos para obtener la forma verdadera del pico de absorción. Hecho ésto se obtiene numéricamente la transformada de Hilbert de los datos de absorción, de acuerdo con la receta descrita con anterioridad para obtener los datos del perfil de dispersión. Finalmente se normalizan los datos de absorción y de dispersión. Esto se hace dividiendo ambos conjuntos de datos entre el máximo valor de la absorción consistente con el criterio para elegir $v_{\max }$, como se estableció antes. En esta forma se obtienen los datos DISPA de la línea bajo estudio. Con los datos DISPA se puede ahora trazar la gráfica DISPA de la línea.

La Figura V-2 muestra la gráfica DISPA para la línea experimental de potasio-amonio $y$, para referencia, se muestra también la gráfica DISPA para una lorentziana pura. De la figura se deduce que la línea de potasio-amonio no es simétrica ya que su gráfica DISPA muestra una ligera asimetría con respecto al eje horizontal. 
La figura también muestra que el máximo esta ligeramente corrido hacia arriba y hacia la izquierda y el mínimo hacia abajo y hacia la izquierda, ambos con respecto al máximo y al mínimo del círculo perfecto asociado a la gráfica DISPA de una lorentziana. Esta línea experimental ha sido reportada en la literatura como como una línea con pequeño contenido gaussiano comparado con el lorentziano $[57,58]$. Lo anterior ha sido confirmado por un reciente análisis numérico realizado por Jiménez-Domínguez et al. [26]. Sin embargo las coordenadas DISPA del máximo y del mínimo observados en la Figura V-2, no obedecen el criterio establecido por las expresiones (V-3). Este comportamiento, denominado anómalo si se consideran las expresiones ( $V$ - 3 ), puede ser debido a otro mecanismo que está actuando durante la resonancia y que modifica al perfil de la línea. Este mecanismo puede ser el llamado ensanchamieto por modulación que no se toma en cuenta en el modelo simple de linea espectral propuesto en el capítulo (III) y que está representado por las expresiones (III-77) y (III-78), (cf. [17], p. 119).

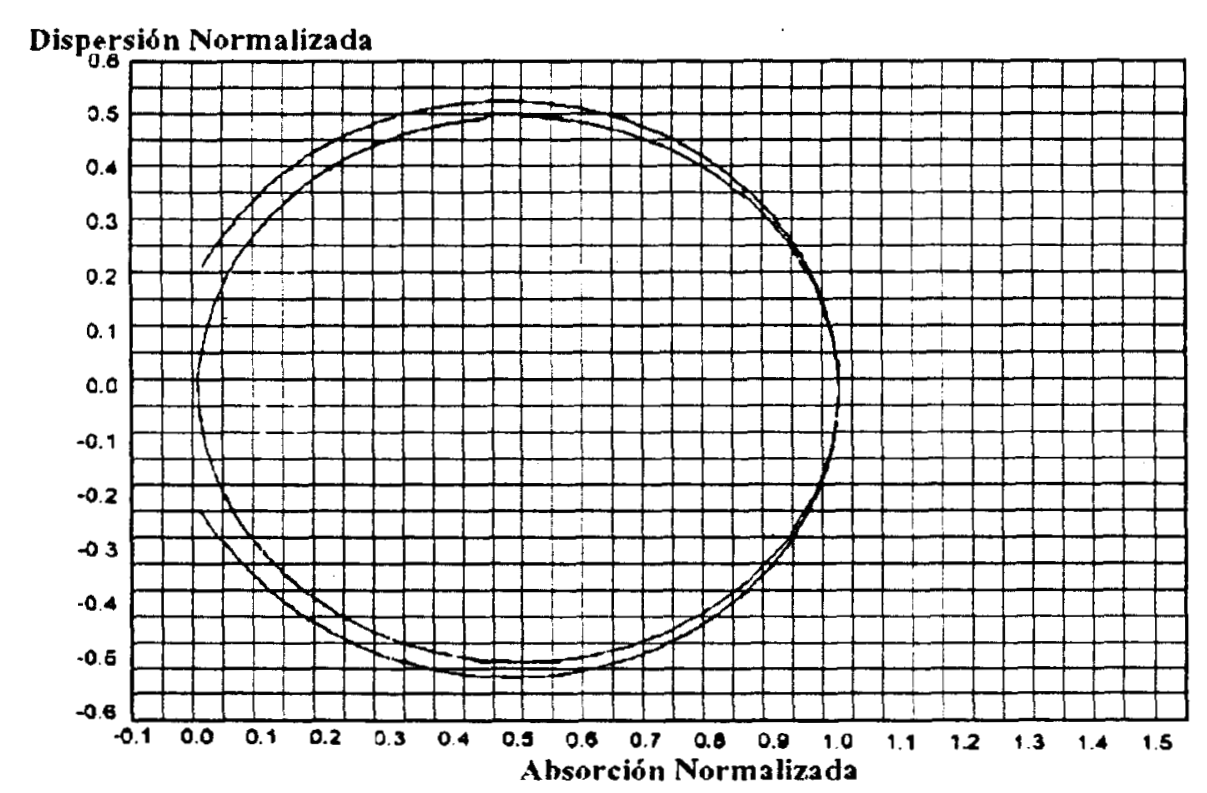

Figura V-2.- La curva exterior muestra la gráfica DISPA de la línea RPE de potasio-amonio. Para referencia, la curva interior muestra el círculo perfecto que corresponde a la gráfica DISPA de un par absorción-dispersión lorentziano. El máximo y el mínimo de la curva exterior muestra un pequeño corrimiento anómalo hacia la 
izquierda en lugar de ser hacia la derecha (ver texto relacionado con las expresiones (V-3)1. Sin embargo los cálculos para encontrar el valor de $u_{\max }$ se realizaron de acuerdo con lo descrito en el texto.

\section{4.- RESULTADOS Y DISCUSION}

A pesar de que la gráfica DISPA de la línea bajo estudio muestra aspectos que no obedecen el criterio establecido por las expresiones (V-3), en esta sección se extraerá alguna información inherente de la línea, empleando el método propuesto en este capítulo.

Los valores resultantes de $\Gamma_{L}$ y $\Gamma_{G}$ obtenidos por medio del presente método se muestran en la Tabla $V-2$, junto con los obtenidos en una operación numérica de ajuste empleando el perfil de Voigt exacto y un perfil de Voigt aproximado [26]. En esta referencia se usaron las derivadas de los perfiles de Voigt, con respecto a la variable de campo magnético, de los perfiles de Voigt aproximado [33] y exacto [II-6]. Los tres conjuntos de valores en esta Tabla se dan en unidades de 0.05 Gauss.

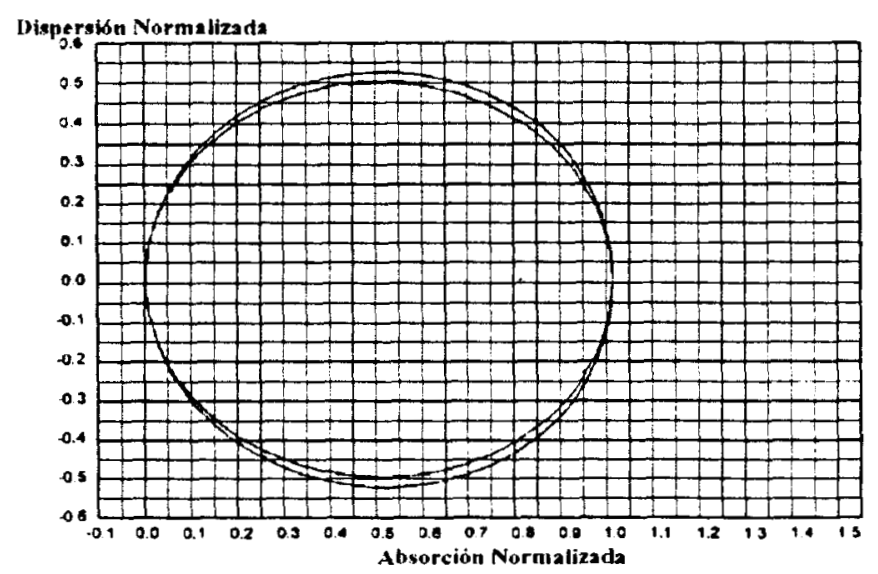

Figura V-3.- La curva externa muestra la gráfica DISPA teórica obtenida mediante el uso de las expresiones (III-76) y (III-78) en las que se introdujeron los valores de $\Gamma_{L}$ y $\Gamma_{G}$ encontrados con el presente método y que están reportados en la segunda columna de la Tabla II. Para referencia la curva interior muestra la gráfica DISPA para una lorentziana. 
Por comparación, la Figura V-3 muestra la gráfica DISPA teórica correspondiente a las expresiones (III-77) y (III-78), alimentadas con los valores de $\Gamma_{L}$ y $\Gamma_{G}$ obtenidos por medio del presente método y reportados en la segunda columna de la Tabla V-2. Para referencia en la Figura V-3 se muestra también la gráfica DISPA de una lorentziana pura (círculo interior).

TABLA V-2

\begin{tabular}{|c|c|c|c|c|}
\hline & $\begin{array}{c}\text { PRESENTE } \\
\text { METODO } \\
(P . M .)\end{array}$ & $\begin{array}{c}\text { EXPRESION } \\
(111-77)\end{array}$ & $\begin{array}{c}\text { EXPRESION } \\
(I I-6)\end{array}$ & $\frac{\Gamma_{P . M}-\Gamma_{(111-77)}(11-6)}{\Gamma_{(111-77)}(11-6)}$ \\
\hline$\Gamma_{L}$ & 3.080 & 3.299 & 3.282 & $-7.0,-6.2$ \\
\hline$\Gamma_{G}$ & 0.529 & 0.606 & 0.665 & $-13.0,-20.5$ \\
\hline
\end{tabular}

TABLA V-2.- Valores de $\Gamma_{L}$ y $\Gamma_{C}$ obtenidos con el presente método (P.M.) (segunda columna). Se muestran también los valores de esos mismos parámetros obtenidos ajustando los datos de absorción con el perfil aproximado (III-77) (tercera columna) y con el perfil de Voigt (II-6) (cuarta columna). La última columna muestra los porcentajes de desviación de los valores en la segunda columna con respecto a los de la tercera y cuarta columna, respectivamente.

El procedimiento aqui empleado supone que la línea es simétrica, de manera que los extremos de la función de dispersión son iguales en valor absoluto y están localizados a la misma distancia del centro de la línea. Sin embargo, este no es el caso general ya que errores de lectura, ruido, presencia de líneas vecinas modifican el perfil de una línea haciéndola no simétrica. Si este es el caso, debe tomarse un promedio de los valores de $\Gamma_{L}$ y $\Gamma_{G}$ obtenidos de cada lado de la línea de dispersión, siempre y cuando el grado de asimetría no sea muy elevado. Si la asimetria de la línea es grande, cualquier estimación de los valores de $\Gamma_{L}$ y $\Gamma_{G}$ por medio del presente método es inütil.

Puede verse de la Tabla V-2 que los valores de los parámetros $\Gamma_{L}$ y $\Gamma_{G}$ obtenidos con el presente método difieren ligeramente de los valores correspondientes obtenidos 
por ajuste de datos con el perfil aproximado (III-76) y con el perfil exacto (II-6), aún en presencia de los corrimientos hacia la izquierda (que deberian ser a la derecha de acuerdo con las expresiones $(V-3))$ que se aprecian en la Figura $V-2$. Sin embargo, al juzgar esos resultados deben enfatizarse y tomarse en cuenta tres aspectos. En primer lugar, el método propuesto en este capítulo es muy simple y no requiere el intenso tratamiento numérico de datos como es el caso de los métodos de ajuste. En segundo lugar, en vista de que la línea RPE de potasio-amonio es sólo levemente gaussiana, el error al calcular la contribución gaussiana $\Gamma_{G}$, comparado con el producido con los métodos de ajuste, puede ser ligeramente mayor al usar un método tan simple como el presente. Este no es el caso para la gran contribución lorentziana $\Gamma_{L}$, cuyos valores obtenidos de las tres maneras tienen una correspondencia muy cercana. En tercer lugar, en vista de que los extremos que aparecen en la gráfica DISPA de la Figura V-2 muestran un corrimiento anómalo hacia la izquierda con respecto a la línea vertical $H_{N}=0.5$, las estimaciones de $\Gamma_{L}$ y $\Gamma_{G}$ pueden ser afectadas por esta fuente de error adicional. No obstante, esta estimación puede ser hecha de acuerdo con el criterio mencionado antes y que está basado en el significado de las expresiones ( $V-3)$.

A continuación se enfatizan tres caracteristicas importantes del presente método:

1).- Los valores de $D_{N}(u, a)$ o $H_{V}(u, a)$ son usados sólamente como referencia. No se realizan cálculos numéricos con ellos.

2).- No se requiere ajuste numérico de curvas para hacer la determinación cuantitativa de los parámetros $\Gamma_{L}$ y $\Gamma_{G}$ de la línea, ya que se dispone de suficiente información analítica para tratar los datos experimentales y hacer los cálculos. En efecto, las expresiones (II-4a), (II-4b) y (V-2) junto con los datos experimentales DISPA constituyen suficiente información para calcular los parámetros de línea $\Gamma_{L} Y \Gamma_{G}$.

31.- En contraposición con las técnicas numéricas las cuales consumen mucho tiempo de máquina, el presente método no requiere estimaciones iniciales de los parámetros de interés. 
Finalmente, con el presente ejemplo se obtuvo buena información acerca de la estructura de la línea RPE de potasio-amonio, aun cuando otros mecanismos perturbadores y modificadores del perfil de línea actuaron durante la resonancia. En particular, un exceso de modulación parece estar presente durante el registro de la línea EPR de potasio-amonio.

En este capítulo se presentó un nuevo método para hacer la deconvolución de una línea lorentziana, levemente convolucionada por una gaussiana. El método requiere solamente la obtención de los datos DISPA de la línea y una expresión analítica que relaciona el valor de a con los puntos en los cuales la dispersión $D(v, a)$ adquiere sus valores extremos. Se presenta como ejemplo la deconvolución del perfil experimental de la línea EPR de una solución de potasio en amonio. La deconvolución se realiza en una forma rápida y sencilla sin necesidad de hacer ajuste numérico.

En el ejemplo se ha visto que el método tiende a subestimar los valores de $\Gamma_{\iota}$ Y $\Gamma_{G}$ por lo que sus resultados pueden ser considerados como una estimación por abajo de las contribuciones lorentziana y gaussiana a la línea, aun en presencia del corrimiento anómalo de los puntos extremos de la gráfica DISPA. Este corrimiento puede deberse a un efecto de modulación el cual no está considerado en el modelo de línea sobre el cual está basado el método. Si bien el método fué aplicado a un caso de espectroscopía RPE, puede ser usado en cualquier espectroscopia en la cual las líneas pueden ser representadas por voigtianas. De los resultados encontrados en este capítulo se puede concluir que el presente método simple tiene el potencial para ser considerado como uno complementario a los procedimientos numéricos existentes para analizar la estructura de líneas voigtianas en varias espectroscopias. Queda por realizar trabajo adicional para considerar los efectos de mecanismos asimetrizadores sobre líneas aisladas no translapadas para prolongar el estudio de estructura de líneas y gráficas DISPA en el caso de lineas asimétricas. Así mismo hace falta evaluar la 
capacidad del presente método en el manejo de casos más complicados tales como el de líneas translapadas. En el siguiente capítulo se manejará la FDP exacta para estudiar líneas asimétricas de absorción y dispersión. 
CAPITULO VI

\section{USO DE LA FUNCION DE DISPERSION DEL PLASMA PARA PROPONER UN MODELO DE LINEA ASIMETRICO Y PARA DESCRIBIR LA SUSCEPTIBILIDAD MAGNETICA COMPLEJA}

\section{1.- INTRODUCCION}

En este último capítulo se resume y generaliza la información expuesta en los anteriores. Por esta razón es que algunos de los conceptos relacionados con el tema central aparecen mencionados nuevamente. Puede decirse, por lo tanto, que el trabajo realizado en esta tesis está representado por este capítulo en el sentido de que los métodos desarrollados en los capitulos anteriores pueden ser generalizados mediante el uso de la FDP exacta. La aplicación se puede hacer a las lineas emitidas por sistemas cuánticos y a aquellos sistemas clásicos en los que la energía oscilatoria interacciona con la materia.

Los niveles cuánticos excitados decaen, en general, a niveles inferiores con la emisión de líneas espectrales de forma lorentziana. Sin embargo, las líneas emitidas por sistemas de interés pueden ser afectadas por perturbaciones internas y/o externas. Si estas perturbaciones tienen un orígen estocástico, pueden ser descritas por una distribución gaussiana y consecuentemente la línea registrada resulta ser la convolución de la forma lorentziana con la distribución gaussiana perturbadora. Este sería el caso de cualquier experimento en el que, por ejemplo, inestabilidades instrumentales, movimientos térmicos, orientaciones azarosas de ejes cristalográficos en muestras de polvos, o algún tipo de mecanismo interno de relajamiento, afectan la forma de las líneas emitidas produciendo un perfil convuelto. Lo anterior es válido también para las líneas de absorción y de dispersión en cualquier espectroscopia (RPE, RMN, Mössbauer, etc.). Se han encontrado varios trabajos en la literatura que están 
dedicados al análisis de esta clase de perfiles espectrales y a su aplicación con el objeto de separar, mediante deconvolución, los dos tipos de perfiles contenidos en las líneas registradas $[3,9,25,57,58,59]$.

En este capitulo se usará la información relacionada con la estructura de líneas espectrales aportada por Jiménez-Dominguez et al [19, 26]. asi como el conocimiento sobre la FDP para analizar la susceptibilidad magnética de un sistema de espines, cuando la excitación resonante de los estados de espin por absorción de microondas (espectroscopía de RPE) o de radiofrecuencia (espectroscopia de RMN), se produce en presencia de mecanismos ensanchadores de línea ya sean internos o externos, de origen aleatorio. Como se ha mencionado antes, la FDP contiene información acerca del relajamiento de ondas que conduce a un perfil lorentziano para el espectro de frecuencias. La FDP también contiene información gaussiana acerca de fenómenos azarosos, por ejemplo movimientos térmicos cuya función de distribución de velocidades altera el perfil lorentziano por convolución. Por otra parte, es sabido que en espectroscopias RPE y RMN, la susceptibilidad compleja se deriva de la solución de las ecuaciones de Bloch para la magnetización transversa en un sistema de espines $[60,61,62]$. Sin embargo, esta solución contiene solamente información del tipo lorentziano, mas no contiene información acerca de fenómenos estocásticos que pudieran tomar lugar en el sistema durante la resonancia. Por ello se mostrará en este capítulo que la susceptibilidad compleja puede ser generalizada para tomar en cuenta un parámetro relacionado con los efectos ensanchadores de orígen aleatorio. Esto se logrará mostrando que la susceptibilidad compleja puede ser escrita en términos de la FDP. En esta forma, las líneas espectrales que se producen durante la excitación resonante de estados de espin pueden ser representadas por perfiles del tipo voigtiano [3], en donde la parte imaginaria de esta susceptibilidad compleja, que representa la absorción de energía electromagnética, adopta una forma voigtiana; la parte real es la transformada de Hilbert de la parte imaginaria y representa la dispersión generalizada. Además, en vista de que en los sistemas de interés siempre están presentes 
mecanismos que producen algún grado de asimetría en líneas que de otra manera serían simétricas, se introducirá un parámetro de asimetría en la FDP para obtener su versión asimetrizada y para generalizar aún más la susceptibilidad compleja. Como antes, en este proceso de generalización no se asignará un significado físico al parámetro de asimetría, ya que distintos mecanismos en las diferentes espectroscopias pueden producir perfiles asimétricos.

Las componentes de absorción y dispersión de la expresión obtenida para la FDP - para la susceptibilidad magnética compleja asimetrizadas, constituyen además un nuevo modelo de línea [63]. Este modelo puede ser útil en muchas espectroscopias para ajustar perfiles experimentales de absorción o dispersión, los cuales muestran en general algún grado de asimetría, para extraer información espectrocópica de interés. Esto se logra a partir de la deconvolución de los perfiles ajustados. El uso de este modelo puede también proporcionar información sobre los fenómenos estocásticos que acompañan los procesos de relajamiento involucrados, asi como sobre mecanismos asimetrizadores de linea que se pueden presentar durante la resonancia.

La expresión para la FDP asimetrizada que se presentará en este capítulo, no solo generaliza el trabajo realizado en capitulos anteriores, sino que también se le usa para obtener una fórmula para la susceptibilidad magnética compleja que toma en cuenta aspectos aleatorios ensanchadores que no son considerados en la solución de las ecuaciones de Bloch [60]. Como se recordará, en el capitulo anterior se asimetrizaron los dos primeros términos del desarrollo asintótico de la FDP.

\section{2.- RELACION ENTRE LA SUSCEPTIBILIDAD MAGNETICA COMPLEJA Y LA FUNCION DE DISPERSION DEL PLASMA}

Es necesario recordar primero que la susceptibilidad compleja de un sistema de spines, en ausencia de mecanismos perturbadores internos o externos de origen aleatorio, como función de un campo magnético externo espacialmente uniforme $h$, puede ser escrita como [60] 


$$
x(h)=x^{\cdot}(h)+i x^{\cdots}(h)
$$

donde $X^{\prime}(h)$ y $X^{\prime \prime}(h)$ son las susceptibilidades en fase $y$ fuera de fase, respectivamente. $X^{\prime}(h)$ corresponde a la parte dispersiva $y x^{\prime}(h)$ a la parte absortiva de la respuesta del sistema de espines a un campo de RF o de microondas aplicado externamente y que oscila en un plano perpendicular al vector del campo magnético uniforme. La ecuación (VI-1) puede ser escrita en términos de la frecuencia $\omega$ del campo de RF o de microondas. Sin embargo, en la práctica es útil explorar con el campo magnético el perfil de las partes absorptiva y dispersiva.

Nótese que la expresión (VI-1) es una función solamente de la variable $h$, asi como de parámetros que caracterizan al campo oscilante y a la relajación lorentziana del sistema; esos parámetros no se considerarán en el tratamiento que sigue. Otros factores asociados con ensanchamientos no lorentzianos de las lineas no se incluyen en (VI-1). La dependencia funcional en $h$ mostrada por la expresión (VI-1), no es suficiente para describir la respuesta del medio a la magnetización si ésta se produce en presencia de perturbaciones aleatorias como por ejemplo movimientos térmicos, orientaciones caóticas de ejes cristalográficos, etc. Esto se debe a que la estructura lorentziana original de (VI-1), que proviene de un sistema de spines mutuamente interactuantes, se convuelve con la distribución gaussiana que representa a esas perturbaciones azarosas, presentes en el sistema cuando ocurre la resonancia [60]. En efecto, estas condiciones perturbadoras no están tomadas en cuenta en las ecuaciones de Bloch, pero pueden ser fácilmente introducidas en (VI-1), de tal modo que la susceptibilidad magnética se vuelve una función más complicada de $h$.

Con el objeto de tomar en cuenta, de una manera fácil y conveniente, los mecanismos perturbadores mencionados antes, es útil recordar que, en presencia de perturbaciones aleatorias descritas por una distribución gaussiana, la amplitud de la absorción de una línea ensanchada es proporcional al perfil de Voigt $y$ se escribe de acuerdo con (II-5) como 


$$
l(u, a)=\frac{1}{\sqrt{2 \pi} \sigma} H(u, a)
$$

donde $\sigma$ es la desviación estandar de la gaussiana ensanchadora y $H(u, a)$, el perfil de Voigt, que está dado por (II-6) y $\backsim$ y $a$ por (II-4a) y (II-4b), respectivamente. Nótese que en espectroscopia sólo los valores $\Gamma_{L}, \Gamma_{G} \geq 0$ son de interés físico, por lo que el intervalo de $c$ es

$$
0 \leq a<\infty \text {. }
$$

La función $H(U, a)$ en (VI-2), puede ser escrita en términos de la FDP, $Z(z)$, como sigue

$$
H(u, a)=\frac{1}{\sqrt{\pi}} \ln Z(i \zeta)
$$

donde $\zeta=a+i v, \quad i=\sqrt{-1},[19]$.

Por otra parte, suponiendo que la absorción $I(U, a)$ en $(V I-2)$ es proporcional a la parte imaginaria de una susceptibilidad compleja generalizada entonces, con la ayuda de (VI-4), se sigue que

$$
\operatorname{lm} x(u, a)=x_{0} \ln Z(i \zeta)
$$

donde $x_{0}$ es una constante real que representa una susceptibilidad estática. Para los propósitos del presente capítulo, se puede demostrar que (VI-5) puede ser escrita en la siguiente forma ligeramente distinta pero un poco más sencilla

$$
\operatorname{Im} \chi(u, a)=\chi_{0} \operatorname{Im} Z(z),
$$

donde

$$
z=v+i a
$$

y

$$
Z(z)=\frac{1}{\sqrt{\pi}} \int_{-\infty}^{\infty} \frac{e^{-y^{2}} d y}{y-z}
$$


es la FDP [4] la cual puede ser calculada con alta precisión empleando un algoritmo desarrollado por Gautschi [20]. Sus partes real e imaginaria se transcriben de (II-42):

$$
\begin{aligned}
& \operatorname{ReZ}(u, a)=\frac{-1}{\sqrt{\pi}} \int_{-\infty}^{\infty} \frac{e^{-y^{2}}(u-y) d y}{(u-y)^{2}+a^{2}} . \\
& \operatorname{lm} Z(u, a)=\frac{a}{\sqrt{\pi}} \int_{-\infty}^{\infty} \frac{e^{-y^{2}} d y}{(u-y)^{2}+a^{2}} .
\end{aligned}
$$

El lado izquierdo de (VI-6) es la parte absortiva de la susceptibilidad compleja escrita en términos de la variable $v$ y del parámetro $a$.

Puesto que la absorción y la dispersión de energía deben tomar lugar bajo las mismas condiciones perturbadoras, en lo que sigue se obtendrá una relación análoga a (VI-6), entre las partes reales de $x y$ de $Z$. Para este fin, se hará uso de de las relaciones de Kramers-Krönig entre las partes real e imaginaria de la susceptibilidad transversa, asi como de las propiedades analíticas de la FDP, $Z(z)$, en el plano complejo. Además, puesto que las relaciones de Kramers-Krönig se cumplen sólamente para sistemas lineales, el desarrollo se dedicará a este tipo de sistemas; dicho de otra manera: no se consideran efectos de saturación. Nótese, por otra parte, que las relaciones de Kramers-Krönig tienen sentido para argumento real en la función compleja $x(v)$, es decir, $a=0$ en la variable compleja $z=v+i a$. Ver, por ejemplo, (III-7). Sin embargo, para este caso particular el perfil (VI-2) es puramente gaussiano (ver la definición de a dada por (II-4b)). Para el propósito de esta parte del capítulo, por lo tanto, es necesario manejar expresiones que sean lorentzianas cuando el argumento de la FDP sea puramente real. Entonces, con el objeto de obtener un perfil puramente lorentziano consistente con la solución de las ecuaciones de Bloch, deben hacerse las siguientes transformaciones en la variable compleja (VI-7). En lugar de " y $a$. debe escribirse respectivamente

$$
u=\frac{v(\ln 2)^{1 / 2}}{a}
$$




$$
\alpha=\frac{\ln 2}{a}
$$

Substituyendo (II-4a) y (II-4b) en (VI-11) y (VI-12), las nuevas variables $u$ y $a$ se escriben ahora respectivamente como

$$
u=\frac{2\left(h-h_{0}\right)(\ln 2)^{1 / 2}}{\Gamma_{L}}
$$

y

$$
\alpha=\frac{\Gamma_{c}(\ln 2)^{1 / 2}}{\Gamma_{L}}
$$

por lo que la nueva variable compleja es:

$$
\because \text { nueva }=u+i \alpha
$$

Obsérvese de los pares de variables (II-4a), (II-4b) y (VI-13), (VI-14) que los papeles de los parámetros de anchura $\Gamma_{L} Y \Gamma_{G}$ han sido intercambiados. Obsérvese también que debido a esas transformaciones, un perfil puramente lorentziano se caracteriza ahora por $\alpha=0$.

Escribiendo las variables $(v, a)$ en términos de las variables $(u, \alpha)$ y substituyendo el par resultante en (II-6), se obtiene la siguiente forma alterna del perfil de Voigt:

$$
H(u, \alpha)=\frac{(\ln 2)^{1 / 2}}{\pi} \int_{-\infty}^{\infty} \frac{\exp -\left[\frac{y^{2} \ln 2}{\alpha^{2}}\right] d y}{(u-y)^{2}+\ln 2}
$$

Si bien la expresión (VI-16) mantiene su forma voigtiana, ella es ligeramente más complicada que la expresión (II-6) porque la forma de las variables (II-4a) y (II-4b) con $\Gamma_{G}$ en el denominador es la "algebraicamente" correcta. La complicada forma (VI-16) del perfil voigtiano es el precio que debe pagarse por haber invertido los papeles $\Gamma_{L}$ 
y $\Gamma_{G}$ que dió como resultado al par de definiciones (VI-13) y (VI-14). Nótese que todos los perfiles $H(u, \alpha)$ pueden ser obtenidos a partir de $H(v, a)$ intercambiando los valores de $\Gamma_{L}, \Gamma_{G}$ y viceversa.

De lo dicho anteriormente, la versión puramente lorentziana de (VI-6) es:

$$
\ln x(u)=x_{0} \operatorname{lm} Z(u) \text {. }
$$

De acuerdo con una de las relaciones de Kramers-Krönig, las partes real e imaginaria de $\chi(u)$ estan ligadas por la expresión

$$
\operatorname{Rex}(u)=\frac{1}{\pi} P \int_{-\infty}^{\infty} \frac{\operatorname{lm} x(x) d x}{x-u}
$$

donde $x$ y $u$ son variables reales y la letra " $P$ " escrita antes del signo de integral significa que debe tomarse el valor principal de Cauchy. Substituyendo (VI-17) en (VI-18) se obtiene

$$
\operatorname{Rex}(u)=\frac{X_{0}}{\pi} P \int_{-\infty}^{\infty} \frac{\operatorname{lm} Z(x) d x}{x-u}
$$

Por otra parte, en vista de que la función $Z(\because)$ es analitica en todo el plano complejo incluyendo el eje real $[4,27]$, se sigue que ella satisface las relaciones de Kramers-Krönig:

$$
\begin{aligned}
& \operatorname{Re} Z(u)=\frac{1}{\pi} P \int_{-\infty}^{\infty} \frac{\ln Z(x) d x}{x-u} \\
& \ln Z(u)=-\frac{1}{\pi} P \int_{-\infty}^{\infty} \frac{\operatorname{Re} Z(x) d x}{x-u}
\end{aligned}
$$

como debe ser para funciones que obedecen el principio de causalidad; particularmente en el caso de la FDP la cual es una relación de dispersión. De (VI-19) y (VI-20) se sigue que

$$
\operatorname{Rex}(u)=\chi_{0} \operatorname{Re} Z(u) \text {. }
$$

Combinando (VI-17) y (VI-22) para construir la cantidad compleja $x(u)$, se obtiene la siguiente expresión 


$$
x(u)=x_{0}[\operatorname{Re} Z(u)+i \operatorname{lm} Z(u)]=x_{0} Z(u) .
$$

Puesto que la FDP de argumento real puede ser continuada analíticamente a todo el plano complejo de la variable (VI-15) [27], al hacerlo, se obtiene la siguiente forma generalizada para la susceptibilidad magnética compleja $\chi$, la cual ya incluye el factor de ensanchamiento de orígen estocástico implícito en el parámetro $\alpha$.

$$
\chi(u, \alpha)=x_{0} Z\left(z_{\text {nueva }}\right) \text {, }
$$

0 , puesto que los mismos perfiles pueden ser obtenidos a partir de $Z(\approx)$

$$
x(u, a)=x_{0} Z(z)
$$

Esto muestra que, cuando los procesos de magnetización en un sistema de espines toman lugar en presencia mecanismos aleatorios ensanchadores de linea internos o externos, la susceptibilidad magnética compleja del sistema puede ser escrita en términos de la FDP. Además los perfiles de absorción y dispersión contenidos en la expresión (VI-24) pueden ser útiles en muchas espectroscopias para ajuste de líneas experimentales. Este es el caso cuando el espectroscopista puede ajustar con voigtianas los perfiles experimentales de absorción y/o de dispersión, con el objeto de encontrar sus contenidos lorentziano y gaussiano respectivamente.

En forma similar, en vista de que la FDP incluye en forma natural un parámetro estocástico ensanchador de lineas, los perfiles de absorción y dispersión en otros campos de la ciencia y de la ingeniería pueden ser generalizados y escritos en términos de esta función.

En la siguiente sección se hará una generalización adicional de $Z(z)$ y consecuentemente de $\chi(v, a)$, consistente en la inclusión de un parámetro de asimetría. En esta forma el campo de aplicación de la FDP se puede expandir aún más y aplicarla en varias disciplinas y espectroscopias donde muchas líneas que tienen un alto contenido voigtiano, poseeen algún grado de asimetría. 


\section{3.- UNA GENERALIZACION SENCILLA DE LA FUNCION DE DISPERSION DEL PLASMA PARA INCLUIR UN PARAMETRO DE ASIMETRIA}

En el analisis previo, se ha encontrado una expresión, la (VI-24), mostrando que la susceptibilidad compleja de un sistema de spines, afectado por perturbaciones estocásticas internas y/o externas, puede ser escrita en términos de la FDP. En otras palabras, las partes real e imaginaria de (VI-24), son proporcionales a las respuestas de absorción y dispersión espectroscópicas de un sistema cuando este se encuentra bajo la acción de un campo de RF o de microondas en presencia de un campo magnético externo constante y de mecanismos ensanchadores de línea de orígen aleatorio.

Por otra parte, ocurre frecuentemente que los perfiles experimentales de absorción y dispersión en espectroscopias RPE, RMN, optica, Mössbauer, etc. presentan algún grado de asimetría. El orígen de esta asimetría puede deberse a diferentes mecanismos en cada caso. Sin embargo, para propósitos de ajuste en cualquier espectroscopía, sería conveniente representar empiricamente en una forma analítica sencilla tal carácter asimétrico. En el capitulo IV fué hecho un primer intento para tratar líneas no simétricas por medio de la modificación de perfiles voigtianos, asi como en el trabajo de Jiménez-Domínguez et al. [7], donde fueron considerados los dos primeros términos del desarrollo asintótico de la FDP. En esta sección se hará una generalización de la FDP incorporándole en una forma empírica un parámetro de asimetría. Se hace énfasis, además, que este proceso será realizado en tal forma que se preserve el carácter de relación de dispersión dela FDP asimetrizada; es decir, las partes real e imaginaria de la FDP asimetrizada resultante deben ser la transformada de Hilbert una de la otra.

Se usará una función asimetrizadora muy simple, como se hizo en las referencias [7, 42] y en el capítulo IV, la cual, sin embargo, deberá ser usada con precaución como se indicará más adelante. Esta función es: 


$$
e^{b u}
$$

en la cual no se asignará un significado físico al parámetro de asimetría $b$ ya que la asimetria de una línea espectral pude ser causada por diversos mecanismos.

El parámetro de asimetría $b$ tomará libremente valores positivos $y$ negativos en un intervalo que será encontrado más adelante. La parte absortiva asimetrizada de la FDP $Z(z)$ será escrita simplemente como

$$
\operatorname{lm} Z(u, a ; b)=e^{b u} \operatorname{lm} Z(u, a) .
$$

Como es de esperarse, la función asimetrizadora (VI-25), hace que la línea resultante se corra con respecto a $\operatorname{lm}(v, a)$ la cual está centrada en $v=0$. Sin embargo, si el espectroscopista está interesado solamente en la estructura de la línea pero no en su posición, este corrimiento no es de importancia.

Para completar el par absorción-dispersión, la parte dispersiva asimetrizada de la FDP se obtiene usando la relación de Kramers-Krönig dada por (VI-20):

$$
\operatorname{Re} Z(u, a ; b)=\frac{1}{\pi} P \int_{-\infty}^{\infty} \frac{e^{b v^{\prime}} \operatorname{lm} Z\left(v^{\prime} \cdot a\right) d v^{\prime}}{u^{\prime}-v} .
$$

o sea que $\operatorname{Re} Z(u, a ; b)$ debe ser la transformada de Hilbert de (VI-26). Nótese que, debido a la expresión (VI-24), la operación anterior tambien produce la parte dispersiva asimetrizada de la susceptibilidad magnética compleja.

Con el objeto de manejar la expresión (VI-27), es conveniente substituir en ella la forma explícita de $\operatorname{Im} Z(z)$ dada por (VI-10). Hecho esto, se obtiene la siguiente expresión para la parte real de la FDP generalizada:

$$
\operatorname{Re} Z(u, a ; b)=\frac{a}{\pi^{3 / 2}} P \int_{-\infty}^{\infty} \int_{-\infty}^{\infty} \frac{e^{-y^{2}} e^{b u^{\prime}} d y d u^{\prime}}{\left(u^{\circ}-u\right)\left[\left(u^{\circ}-y\right)^{2}+a^{2}\right]}
$$

Para continuar, es conveniente hacer primero la integral sobre " en (VI-28). Para este fin se realizará una integración compleja por medio de un procedimiento similar al usado para efectuar la integral (IV-15) y después se tomará la parte real del resultado, 
puesto que el valor principal de Cauchy es una cantidad real. Como en el caso de (IV-15), para la presente integración compleja, se elegirá un contorno en el plano complejo de $v^{\prime}$ que incluya al eje real $y$ un semicírculo en el semiplano superior. La parte del contorno a lo largo del eje real se deformará para excluir el polo en $v^{\prime}=v$.

El polo contenido dentro del contorno es entonces $v^{\prime}=y+i a$. Para ilustrar este contorno de integración véase la Figura III-3 en la que sólo es necesario excluir el polo sobre el eje real por medio de una pequeña semicircunferencia recorrida en el sentido de las manecillas del reloj. En seguida se impondrá la siguiente condición sobre los valores positivos de $b$, con el objeto de impedir que la integral sobre el semicirculo en el infinito diverja conforme $\left|u^{\prime}\right| \rightarrow \infty$.

$$
0 \leq b<1
$$

Bajo esta condición, la parte principal de (VI-28) se escribe simbólicamente como

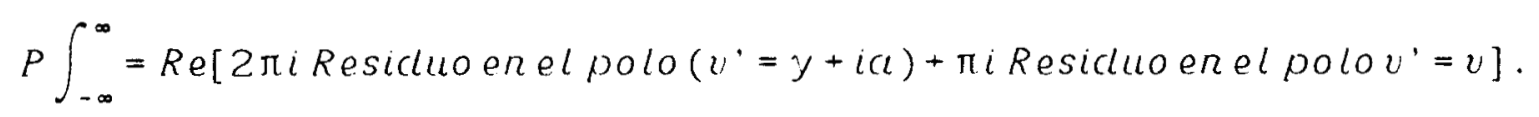

Después de ejecutar la integración sobre $v^{\prime} \mathrm{y}$ hacer uso de la expresión (VI-10), se obtiene la siguiente función compleja antes de tomar la parte real

$$
\begin{gathered}
F(u, a ; b)=\frac{-\cos (a b)}{\sqrt{\pi}} \int_{-\infty}^{\infty} \frac{e^{-\left(y^{2}-b y\right)}(u-y) d y}{(u-y)^{2}+a^{2}}+ \\
+\frac{a \operatorname{Sen}(a b)}{\sqrt{\pi}} \int_{-\infty}^{\infty} \frac{e^{-\left(y^{2}-b y\right)} d y}{(u-y)^{2}+a^{2}}+ \\
+i\left[\frac{a e^{b u}}{\sqrt{\pi}} \int_{-\infty}^{\infty} \frac{e^{-y^{2}} d y}{(u-y)^{2}+a^{2}}-\frac{a \cos (a b)}{\sqrt{\pi}} \int_{-\infty}^{\infty} \frac{e^{-\left(y^{2}-b y\right)} d y}{(u-y)^{2}+a l^{2}}+\right. \\
\left.-\frac{\operatorname{sen}(a b)}{\sqrt{\pi}} \int_{-\infty}^{\infty} \frac{e^{-\left(y^{2}-b y\right)}(u-y) d y}{(u-y)^{2}+a l^{2}}\right] .
\end{gathered}
$$

Análogamente al tratamiento del caso de la asimetrzación del perfil aproximado de Voigt en el capitulo IV, nótese que si se pone $b=0$ en la expresión (VI-30), la parte imaginaria se cancela y se recupera la forma original de $I m Z(v, c)$ dada por 
la expresión (V1-10). En vista de que sólo debe mantenerse la parte real de (VI-30), después de un poco de álgebra se obtiene la siguiente expresión para la parte real de la FDP asimetrizada

$$
\operatorname{Re} Z(v, a ; b)=e^{\frac{b^{2}}{4}}\left[\frac{-\cos (a b)}{\sqrt{\pi}} \int_{-\infty}^{\infty} \frac{e^{-(y-b / 2)^{2}}(u-y) d y}{(u-y)^{2}+a^{2}}+\frac{a \operatorname{Sen}(a b)}{\sqrt{\pi}} \int_{-\infty}^{\infty} \frac{e^{-(y-b / 2)^{2}} d y}{(u-y)^{2}+a^{2}}\right],
$$

la cual, después de hacer el cambio de variable $y=x+b / 2$ y usar las definiciones (VI-9) y (VI-10), se escribe como

$$
\operatorname{Re} Z(u, a ; b)=e^{\frac{0^{2}}{4}}[\operatorname{Cos}(a b) \operatorname{Re} Z(u-b / 2, a)+\operatorname{Sen}(a b) \operatorname{lm} Z(u-b / 2, a)] \text {, }
$$

en la cual ambos perfiles dentro del paréntesis cuadrado, están corridos por una cantidad $b / 2$. El sentido de corrimiento será determinado por el signo que asuma el parámetro $b$. Por lo tanto, la expresión para la FDP asimetrizada es

$$
Z(u, c ; b)=\operatorname{Re} Z(u, a ; b)+i I m Z(u, a ; b)
$$

donde $\operatorname{Re} Z(v, a ; b)$ y $\operatorname{Im} Z(v, a ; b)$ están dadas por las expresiones (VI-31) y (VI-26), respectivamente. Como consecuencia, la expresión para la susceptibilidad compleja generalizada es

$$
x(u, a ; b)=x_{0} Z(u, a ; b)
$$

en la cual el intervalo de $b$ será definido a continuación.

Como fué mencionado antes, debe tenerse cuidado al considerar los valores del parámetro b puesto que no hay garantía de que el perfil representado por la expresión (VI-26) tenga extremos para todos los valores de $b$ contenidos en el intervalo (VI-29) y los correspondientes valores negativos. Este problema será resuelto demandando que los extremos de (VI-26) existan. Tomando la derivada de (VI-26) con respecto a la variable $v$ e igualándola a cero, los extremos de (VI-26) existen si se cumple la siguiente condición 


$$
a^{2} b^{2} \leq 1 .
$$

la que, como era de esperarse, es igual a la exigida para los mismos fines en el caso de la asimetrización del perfil aproximado de Voigt, véase (IV-18).

Sin embargo, como el parámetro a puede tomar valores positivos solamente, de acuerdo con la expresión (VI-3), esta condición puede ser escrita como:

$$
a|b| \leq 1 \text {. }
$$

Esta condición indica que los parámetros $a$ y $b$ no son independientes uno del otro. Por ejemplo, para valores grandes de a (contenido gaussiano muy pequeño), sólo se permiten valores muy pequeños de $b$ (un grado muy pequeño de asimetría). La forma de los perfiles se detetrmina de acuerdo con el siguiente esquema

$$
\begin{aligned}
& b>0 \text { : asimetria por el lado derecho. } \\
& b=0: \text { per fil simétrico. } \\
& b<0 \text { : asimetria por el lado iz:quierdo. }
\end{aligned}
$$

Nótese que la condición (V1-34) sobre los parámetros $a$ y $b$ es de un carácter general para lineas espectrales voigtianas cuya asimetría se origina en factores externos no especificados. De (VI-34) es posible deducir que el grado de asimetría inducido sobre la forma de línea original no puede exceder su deformación, representada por el ensanchamiento gaussiano dividido entre $\Gamma_{L} \sqrt{\ln 2}$, sin destruir toda la información espectral.

Si se requiere hacer una estimación de la amplitud del intervalo para $|b|$ en la expresión (VI-34), deben hacerse las siguientes consideraciones. La condición (VI-34) surge debido a que las integrales involucradas se ejecutan en el intervalo $-\infty<v<\infty$ en el cual la expresión (VI-26) debe tener valores extremos. Sin embargo en una situación experimental, solamente un intervalo finito de $v$ es suficiente para describir un perfil (ver por ejemplo las Figuras VI-1 y VI-2); el más amplio intervalo requerido 
es para la lorentziana pura la cual tiene amplias alas y valores grandes del parámetro a. Por ejemplo, para $a=10$, la componente gaussiana es tan pequeña que el perfil puede considerarse lorentziano para propósitos prácticos. Por lo que si este valor de a se introduce en (VI-34), la estimación para la amplitud del intervalo de asimetría es

$$
|6| \leq 0.1 \text {. }
$$

Debe mencionarse que, para propósitos de ajuste y de análisis de estructura de líneas, ni el factor de escala $e^{b^{2 / 4}}$ ni el corrimiento de la variable $\iota$ que aparecen en la expresión (VI-31) son necesarios por lo que, para esas tareas, la expresión (VI-31) que representa la dispersión, puede ser escrita como

$$
\operatorname{Re} Z(u \cdot a: b)=\operatorname{Cos}(a b) \operatorname{Re} Z(u \cdot a)+\operatorname{Sen}(a b) \operatorname{lm} Z(u \cdot a) \cdot \quad(V /-36)
$$

mientras que la expresión (V1-26) puede ser usada como tal, para hacer ajustes.

Un aspecto interesante que debe ser resaltado de las expresiones (VI-26) y (VI-36) es la simplicidad con la que una función tan complicada como la FDP permite ser asimetrizada por medio de la función (VI-25).

En la Figura VI-1 se muestran gráficas para el perfil de absorción representado por la expresión (VI-26) para $\Gamma_{L}=10, \Gamma_{G}=9$ y $b=0, \pm 0.1$. En la Figura VI-2 se muestran gráficas para los perfiles de dispersión representados por la expresión (VI-36) para los mismos valores de los parámetros $\Gamma_{L}, \Gamma_{G}, b$. Todas las gráficas en estas figuras tienen la intensidad normalizada. En estas figuras se manifiesta claramente el efecto producido por el parámetro $b$ sobre los perfiles de absorción y dispersión. Nótese también que la amplitud del intervalo del parámetro $b$. dado por la expresión (VI-35), es mayor que la del intervalo indicado por la expresión (IV-20). Esto significa que las expresiones para el par absorción-dispersión representado por las expresiones (VI-26) y (VI-36), escritas en términos de la FDP exacta, son capaces de manejar grados mayores de asimetría. 


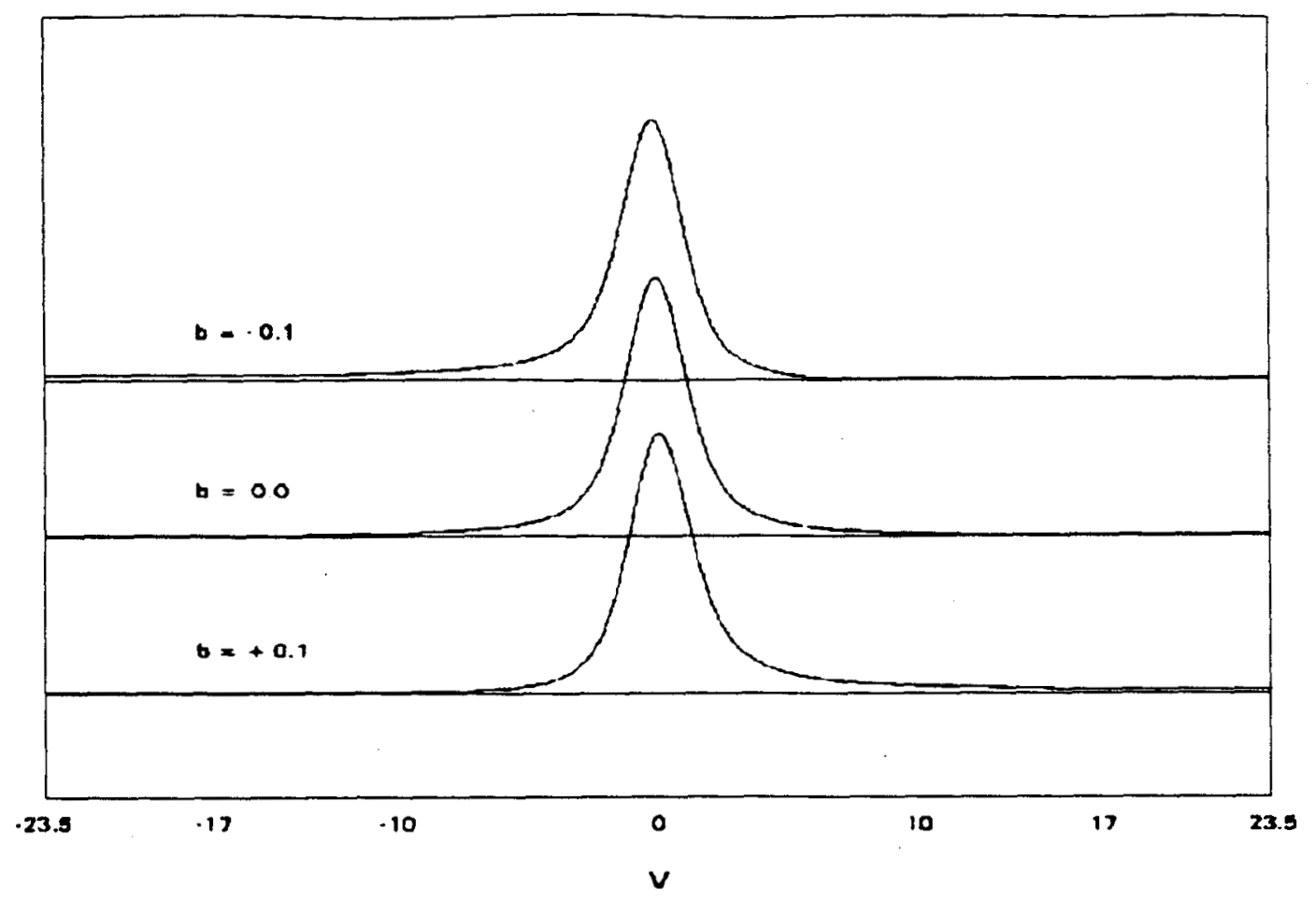

Figura VI-1.- Perfiles de absorción representados por la expresión (VI-26) para $\Gamma_{L}=10, \Gamma_{C}=9$ y $b=0, \pm 0.1$. Se manifiesta claramente el efecto de asimetría causado por el parámetro $b$. Las dimensiones de $\Gamma_{L} y \Gamma_{G}$ dependen del tipo de espectroscopía considerado: canales, frecuencia, campo magnético, etc.

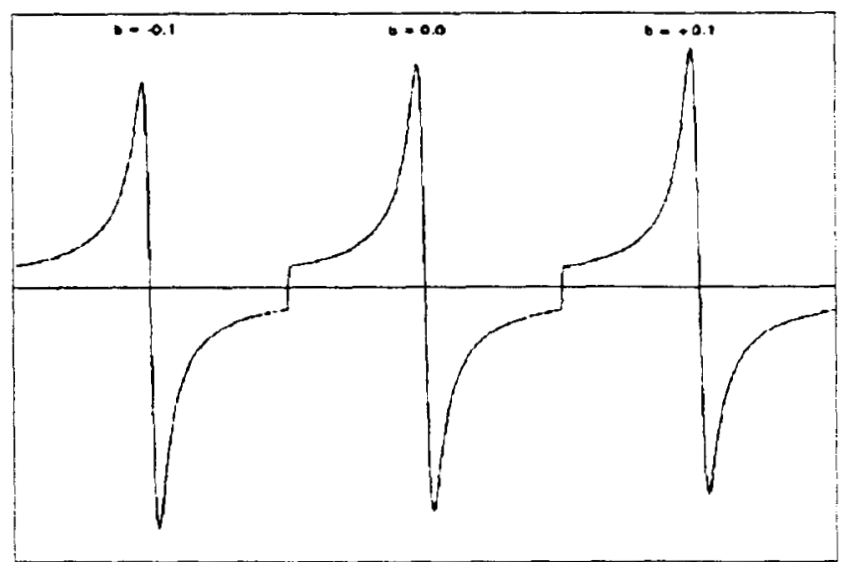

Figura VI-2.- Perfiles de dispersión representados por la expresión (VI-36) para $\Gamma_{L}=10, \Gamma_{C}=9$ y $b=0,0.1$. . Se manifiesta claramente el efecto de asimetría causado por el parámetro $b$. Las dimensiones de $\Gamma_{L} y \Gamma_{C}$ dependen del tipo de espectroscopía considerado: canales, frecuencia, campo magnético, etc. 


\section{4.- APLICACION DEL MODELO A UNA LINEA EXPERIMENTAL}

En esta sección se presentará un ejemplo sobre el uso del modelo basado en la FDP, para ajustar una linea espectral experimental. Para esto se usará una línea RPE de absorción de una solución de potasio en amonio tomada de la literatura $[57,58]$. Ahora bien, como se trata de una línea de absorción y no de dispersión, para realizar el ajuste se debe usar la parte imaginaria asimetrizada de la FDP dada por la expresión (VI-26). Sin embargo, en vista de que es conocido que la línea elegida tiene un contenido gaussiano pequeño (un valor grande del parámetro a) $[57,58$ ], en lugar de usar la expresión (VI-26) se usará una forma aproximada de ella para valores grandes de $a$. Esta forma está representada por la expresión (IV-21) y será suficiente para ajustar la línea.

Además, para complementar el ejemplo, se usará tambien el modelo de línea propuesto por Losev [42] para ajustar la línea experimental, con el objeto de hacer una comparación gráfica entre ambos ajustes.

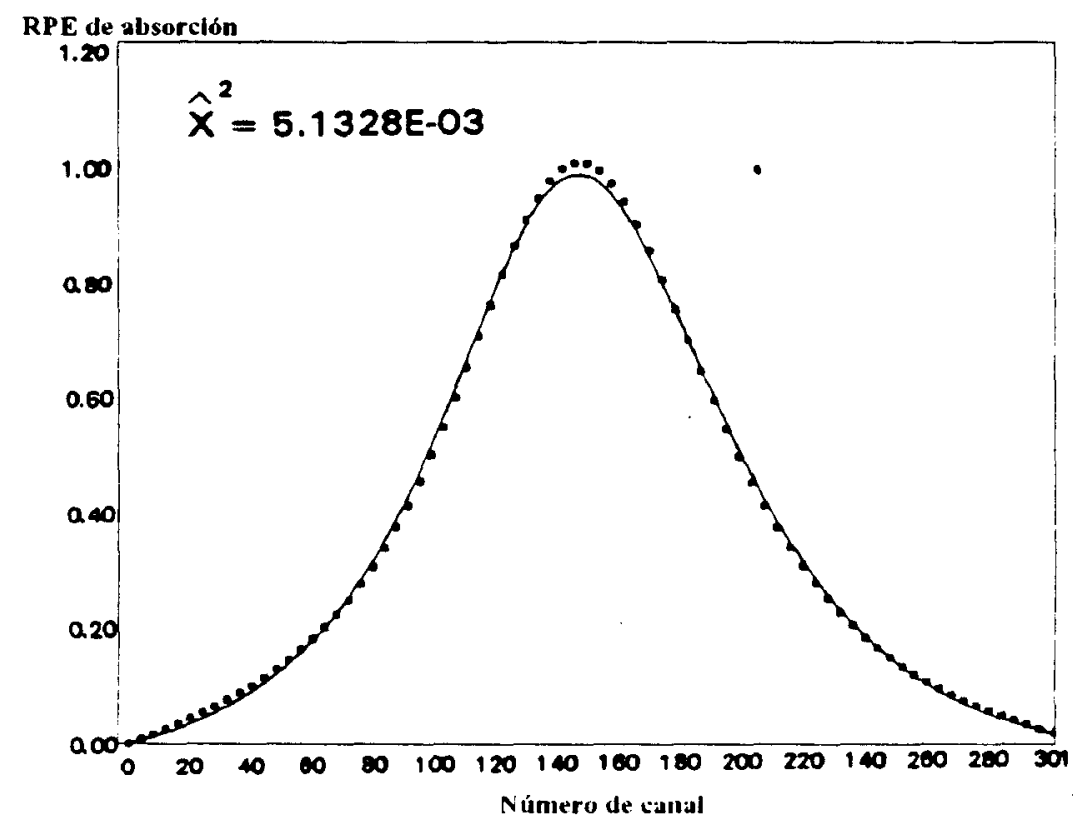

Figura VI-3.- La línea contínua representa el ajuste de datos con el modelo de línea de Losev [42] a la línea experimental de potasio-amonio tomada de la literatura. 


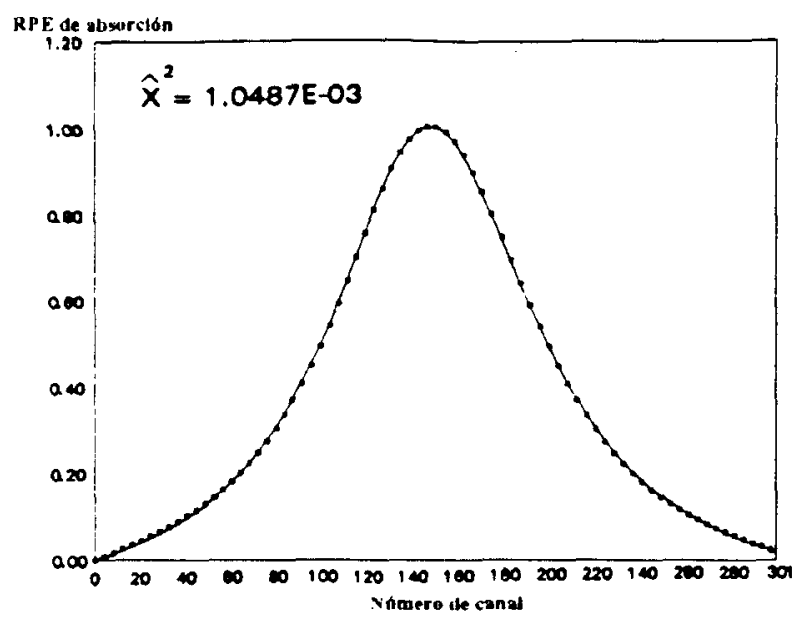

Figura VI-4.- La linea continua representa el ajuste de datos con el modelo de línea representado por la expresión (IV-21), a la línea experimental de potasio-amonio tomada de la literatura.

Las Figuras VI-3 y VI-4 muestran, respectivamente, el mejor ajuste a los puntos experimentales, obtenido con el modelo de Losev y con el modelo basado en la FDP. Se puede ver de estas figuras que el mejor ajuste se obtiene con el modelo basado en la FDP. Los valores de los parámetros $\Gamma_{L}, \quad \Gamma_{G}$ encontrados por medio del ajuste con el modelo basado en la FDP son: $\Gamma_{L}=3.418, \Gamma_{G}=0.582$, en unidades de 0.05 Gauss. El valor del parámetro adimensional de asimetría $b$ es $1.3513 \times 10^{-3}$. Los valores para $\Gamma_{L}, \Gamma_{G}$ aquí obtenidos son congruentes con los obtenidos en las referencias [8] y [26,], donde se usó la versión simétrica aproximada (III-76), de la expresión (VI-26). Es necesario hacer notar, sin embargo, que los valores de $\Gamma_{L}$ y $\Gamma_{C}$ obtenidos en este ejemplo, difieren un poco de los obtenidos en [8] y [26], i. e. el primero es más grande y el segundo es más pequeño, respectivamente, que los citados. Pero esto no es sorprendente puesto que el factor de asimetría incorporado en la FDP permite hacer una descripción más realista de la estructura de la línea. En efecto, la asimetría de la línea es considerada en el nuevo modelo en forma independiente de su naturaleza gaussiana y lorentziana. Debe notarse tambien que si bien el ajuste de los puntos experimentales usando el modelo de Losev no es malo, 
de todas maneras se logra una mejora significativa cuando se usa el modelo propuesto en este capítulo, con la ventaja adicional de obtener información sobre la estructura de la línea a través de sus anchos lorentziano y gaussiano.

En este capítulo ha sido mostrado que cuando el fenómeno de resonancia magnética está acompañado por procesos perturbadores de origen estocástico, la susceptibilidad magnética compleja puede ser expresada en términos de la conocida FDP. En esta forma la susceptibilidad original de Bloch la cual tiene una naturaleza lorentziana, adopta una voigtiana. Además, se introdujo un parámetro de asimetría en la FDP, y consecuentemente en la susceptibilidad compleja, para simular el efecto de mecanismos asimetrizadores de línea que pueden ocurrir en la muestra durante la resonancia. Esta generalización de la FDP y de la susceptibilidad magnética compleja produce un nuevo modelo espectroscópico de línea para absorción y dispersión, basado en un perfil voigtiano. Este nuevo modelo está representado por las expresiones (VI-26) y (VI-36) y puede ser útil para ajustar líneas espectrales asimétricas y obtener información valiosa sobre anchuras lorentziana y gaussiana, asi como sobre mecanismos ensanchadores estocásticos y asimetrizadores de líneas. En este capitulo se encontró también una relación importante de carácter general entre los parámetros $a$ y $b$ de la cual se puede deducir que el grado de asimetría inducido sobre la línea por factores no especificados, no puede exceder su ensanchamiento gaussiano normalizado a $\Gamma_{L} \sqrt{\ln 2}$, sin obstruir toda información espectral. Debe mencionarse que este concepto era conocido intuitivamente en espectroscopia y ahora se confirma formalmente en este tratamiento por medio de la expresión (VI-34). Este concepto se manifestó ya en el capítulo IV al tratar el problema de la asimetrización del perfil aproximado de Voigt, ver Ec (IV-18), sin embargo en este capitulo dicho concepto se refuerza al tratar el problema de la asimetrización de la FDP exacta.

Es necesario mencionar una vez más que los modelos asimétricos presentados en este capitulo y en el IV, pueden ser usados en cualquier espectroscopía para ajustar y analizar líneas experimentales de absorción y dispersión que presenten asimetria. 
Como un último comentario debe enfatizarse que mientras otros modelos de línea en la literatura consisten sólamente del perfil de absorción, los nuevos modelos presentado en este trabajo consiste del par absorción-dispersión obtenido de tal manera que sus miembros forman una relación de dispersión, la cual contiene el concepto físico de causalidad. Este hecho proporciona un soporte formal a los modelos presentados en esta tesis. 


\section{CONCLUSION}

En el protocolo de tesis se describe el programa de la investigación a realizar. El programa se cumplió realizando una investigación basada en una idea general que fué la de extender el dominio de aplicación de la función de dispersión del plasma al campo de la espectroscopía. La meta era proponer un nuevo modelo para representar analíticamente los perfiles de absorción y dispersión que se producen durante la interacción de energía oscilatoria con la materia. Dentro de la misma idea y del mismo campo, se buscaba también escribir la susceptibilidad magnética compleja de un sistema de espines en términos de la misma FDP, con el objeto de tomar en cuenta mecanismos de ensanchamiento de origen aleatorio, aspecto no considerado en las ecuaciones de Bloch.

Esa idea surge de reconocer que el perfil de Voigt, usado desde hace mucho tiempo con buenos resultados en varias espectroscopias para ajustar líneas espectrales de absorción o emisión, es proporcional a la parte imaginaria de la FDP. La parte real de la FDP es proporcional al perfil de dispersión el cual es la transformada de Hilbert del de absorción.

En este trabajo se demostró que la FDP al contener informaciones lorentziana (forma "natural" de línea) y gaussiana (mecanismos perturbadores de orígen aleatorio), puede ser usada para simular y ajustar líneas espectrales experimentales de absorción y dispersión, en varias espectroscopias. En este marco se obtuvieron varios resultados interesantes.

Un primer resultado de la investigación fué proponer un nuevo método para estimar la temperatura y densidad electrónicas de un plasma maxwelliano por medio de la deconvolución de la parte imaginaria de la FDP. En efecto, una onda electromagnética con frecuencia $\omega_{0}$ y de pequeña amplitud que se hace propagar en un plasma, queda sujeta simultáneamente a dos efectos: uno de amortiguamiento 
sobre su amplitud (absorción de la energía de la onda por parte de los electrones) que hace que el espectro de frecuencias de la onda adquiera una forma lorentziana $y$ un efecto de ensanchamiento de este perfil debido al movimiento caótico de los electrones cuyas velocidades están descritas por la distribución de Maxwell. El resultado de ambos efectos es que el espectro de frecuencias de la onda adquiere un perfil que es la convolución de la curva lorentziana con la distribución de tipo gaussiano que caracteriza las velocidades de los electrones en el plasma. Este es el llamado perfil de Voigt. En este contexto la palabra deconvolución significa que el espectroscopista debe determinar los parámetros que caracterizan a los perfiles lorentziano y gaussiano que se convolucionan para formar el perfil de la línea observada.

En ocasiones el espectroscopista conoce a priori que su espectro contiene una mayor contribución lorentziana que gaussiana o viceversa. En esos casos es práctico disponer de formas aproximadas para los perfiles de absorción y de dispersión los cuales permiten una estimación rápida de los parámetros de línea. Parte de los resultados de este trabajo es un nuevo par absorción-dispersión, para el caso de pequeña contribución gaussiana, obtenido bajo la demanda de que éste debe constituir una relación de dispersión; es decir, que los miembros del par satisfagan las relaciones de Kramers-Krönig. Por otra parte, en virtud de que la aparición de líneas espectrales asimétricas es muy frecuente, se introduce un factor de asimetría en este par absorción-dispersión, otra vez bajo la demanda de que las nuevas expresiones constituyan una relación de dispersión. En estas condiciones se obtuvieron nuevas formas de línea asimétricas (del lado derecho o del izquierdo) para simular o ajustar líneas espectrales experimentales. Como un ejemplo de aplicación, se usó el perfil de absorción asimétrico para la determinación de la temperatura de la componente neutra de un plasma de helio producido por microondas. El valor obtenido fué congruente con los valores reportados en la literatura para este tipo de plasmas. 
Otro resultado reportado en este trabajo es un nuevo y sencillo método para deconvolucionar líneas espectrales simétricas con pequeña contribución gaussiana. El método se vale de los datos DISPA de las líneas y su característica principal es que no se basa en técnicas numéricas de ajuste. El método fué probado para deconvolucionar una línea RPE de una solución de potasio en amonio la cual tiene poca contribución gaussiana y un pequeño grado de asimetría. Los resultados fueron congruentes con los obtenidos anteriormente por éste y otros autores.

Finalmente, se maneja la FDP $\sin$ aproximaciones y se le asimetriza por medio de un método similar al anteriormente usado, dando como resultado un nuevo $y$ elegante par absorción-dispersión que también constituye un nuevo modelo de línea espectral. En términos de estas expresiones se escribe la susceptibilidad magnética compleja de un sistema de spines que puede ser de utilidad en espectroscopía RPE. Pero quizá el resultado más importante en este trabajo es el de dar una explicación teórica a un concepto ya conocido en la práctica espectroscópica de que el grado de asimetría inducido sobre un perfil de línea por factores no especificados, no puede sobrepasar su anchura gaussiana sin obstruir toda información espectral.

Estos resultados pudieron ser logrados merced a un tratamiento de tipo general, basado en la exigencia de que cada par absorción-dispersión fuera obtenido de tal manera que constituyera una relación de dispersión.

De acuerdo con lo descrito, resulta pertinente mencionar que los objetivos planteados en el protocolo de tesis fueron superados. Se publicaron tres artículos en revistas de alto impacto $y$ se presentaron dos trabajos en sendos congresos internacionales. 


\section{APENDICE \\ SOLUCION DE VLASOV}

El sistema de ecuaciones (II-30) y (II-31) fué resuelto por Vlasov [22] para el caso de longitudes de onda grandes y en forma general por Landau [23], como un problema de valor inicial. El método de Landau se basa en el empleo de una variable compleja para representar la frecuencia de oscilación y de la transformada de Fourier-Laplace de las funciones $f_{1}(\overrightarrow{\mathbf{r}}, \overrightarrow{\mathbf{v}}, t)$ y $\phi_{1}(\overrightarrow{\mathbf{r}}, t)$.

Siguiendo el tratamiento de Landau se definen las siguientes transformadas espaciales de Fourier:

$$
\begin{aligned}
& f_{1}(\overrightarrow{\mathbf{k}}, \overrightarrow{\mathbf{v}}, t)=\int f_{1}(\overrightarrow{\mathbf{r}}, \overrightarrow{\mathbf{v}}, t) e^{i \overrightarrow{\mathbf{k}} \cdot \vec{r}} d \overrightarrow{\mathbf{r}}, \\
& \phi_{1}(\overrightarrow{\mathbf{k}}, \overrightarrow{\mathbf{v}}, t)=\int \phi_{1}(\overrightarrow{\mathbf{r}}, \overrightarrow{\mathbf{v}}, t) e^{i \overrightarrow{\mathbf{k}} \cdot \vec{r}} d \overrightarrow{\mathbf{r}},
\end{aligned}
$$

y sus transformadas de Laplace:

$$
\begin{array}{ll}
f_{1}(\overrightarrow{\mathbf{k}}, \overrightarrow{\mathbf{v}}, s)=\int_{0}^{\infty} e^{-s t} f_{1}(\overrightarrow{\mathbf{k}}, \overrightarrow{\mathbf{v}}, t) d t, & \operatorname{Re}[s]>x_{0},(A-3) \\
\phi_{1}(\overrightarrow{\mathbf{k}}, \overrightarrow{\mathbf{v}}, s)=\int_{0}^{\infty} e^{-s t} \phi_{1}(\overrightarrow{\mathbf{k}}, \overrightarrow{\mathbf{v}}, t) d t, & \operatorname{Re}[s]>x_{0} . \quad(A-4)
\end{array}
$$

En $(A-3,4) x_{0}$ representa una línea vertical paralela al eje imaginario, en el plano complejo $s$, situada a la derecha de todas las singularidades de $f(\vec{k}, \vec{v}, s)$, ver Figura A-1.

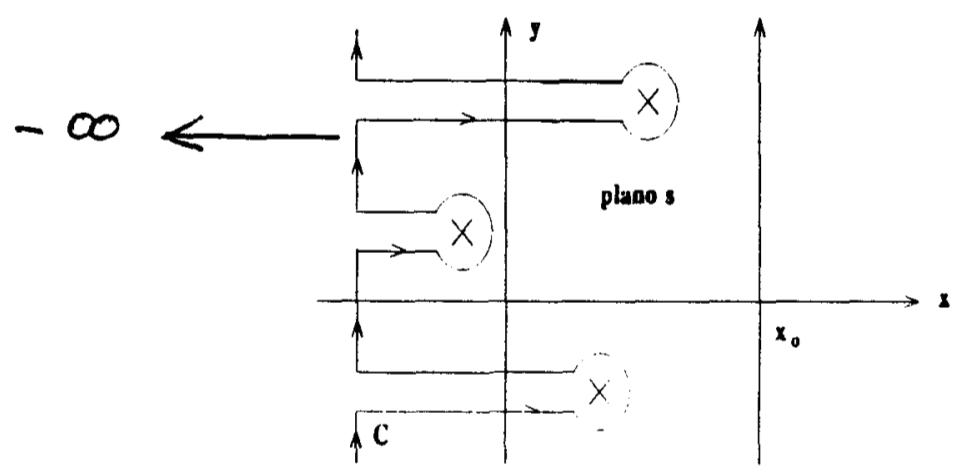

Figura A-1.- La línea vertical $x=x_{0}$ es el contorno de Bromwich a la derecha de todas las singularidades de $\phi_{1}(k, s)$ en el plano complejo $s$. El desplazamiento del polo 
en la Fig. A-2 para realizar la integración sobre la variable $u$ a lo largo del contorno de Landau, produce la continuación analítica de $\phi_{1}(k, s)$ en la zona comprendida entre el contorno $x=x_{0}$ y el contorno $c$ que indenta a todos los polos de $\phi_{1}(k, s)$.

Las transformadas de Fourier-Laplace de (II-30) y (II-31) son, entonces:

$$
\begin{gathered}
(s+i \overrightarrow{\mathbf{k}} \cdot \overrightarrow{\mathbf{v}}) f_{1}(\overrightarrow{\mathbf{k}}, \overrightarrow{\mathbf{v}}, s)-g(\overrightarrow{\mathbf{k}}, \overrightarrow{\mathbf{v}})=\frac{i e}{m} \phi_{1}(\overrightarrow{\mathbf{k}}, s) \overrightarrow{\mathbf{k}} \cdot \nabla_{v} f_{0}(\overrightarrow{\mathbf{v}}),(A-5) \\
k^{2} \phi_{1}(\overrightarrow{\mathbf{k}}, s)=+\pi e \int f_{1}(\overrightarrow{\mathbf{k}}, \overrightarrow{\mathbf{v}}, s) d \overrightarrow{\mathbf{v}}, \quad(t-6)
\end{gathered}
$$

donde:

$$
g(\vec{k}, \vec{v})=f_{1}(\vec{k}, \vec{v}, t=0),
$$

es la transformada de Fourier, de la perturbación inicial en la función de distribución.

Combinando $(A-5)$ y $(A-6)$, se obtiene la transformada de Fourier-Laplace del potencial de perturbación $\phi_{1}(\overrightarrow{\mathbf{r}}, t)$ :

$$
\phi_{1}(\vec{k}, s)=\frac{-i \frac{4 \pi e}{k^{2}} \int_{c} \frac{g(k, u)}{k u-i s} d u}{1-\frac{4 \pi e^{2}}{m k} \int_{c} \frac{d F_{0}(u) / d u}{k u-i s} d u}, \quad(A-8)
$$

donde el eje $\because y$ el vector $\vec{k}$ se han elegido codireccionales; además

$$
g(k, u)=\int g(\vec{k}, \vec{v}) d v_{x} d v_{y}
$$

y

$$
F_{0}(u)=\int f_{0}(\vec{v}) d v_{x} d v_{y} .
$$

En las integrales de (A-8), el contorno de integración $c$ en el plano $u$, llamado contorno de Landau, se muestra en la Figura A-2. 


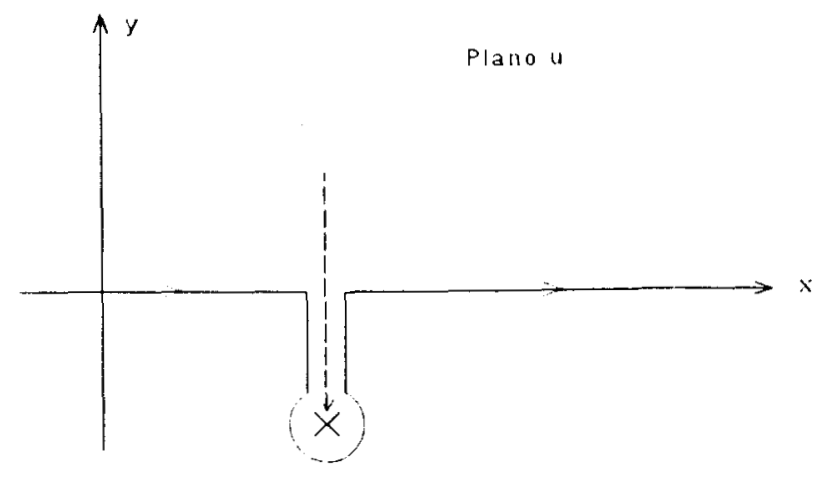

Figura A-2.- Contorno de Landau en el plano complejo «

Este contorno en el plano complejo « tiene la virtud de realizar la continuación analítica de $\phi_{1}(k, s)$ en el plano complejo $s$, desde $\operatorname{Re}[s]>x_{0}$, como se indica en $(A-3,4)$ hasta $\operatorname{Re}[s]<0$ en tal forma que queden indentados todos los polos de $\phi_{1}(k, s)$. En estas condiciones la solución $\phi_{1}(k, t)$ que normalmente tomaría la forma

$$
\phi_{1}(k, t)=\frac{1}{2 \pi i} \int_{B r o \text { mwich }} \phi_{1}(k, s) e^{s t} d s, \quad \operatorname{Re}[s]>x_{0}, \quad(A-9)
$$

se puede escribir equivalente y convenientemente como

$$
\phi_{1}(k, t)=\sum_{i} e^{s_{i} t} \text { Residuo }\left[\phi_{1}(k, s)\right]_{s=s_{i}}, \quad(A-10)
$$

donde $s_{i}$ representa a los polos de $\phi_{1}(k, s)$ en el plano complejo $s$. En la Figura A-1 se muestran el contorno de Bromwich y el que, despues de la continuación analítica de $\phi_{1}(k, s)$, se usa para manejar la expresión (A-10).

Para determinar cómo las integrales en (A-8) contribuyen a las singularidades de $\phi_{1}(k, s)$, considérese primero el numerador de (A-8). Si se supone que la perturbación 
inicial, $g(k, u)$, no es singular en el eje real, el numerador de (A-8) contribuye sólo con polos en los que él decae en el tiempo. En efecto, si $R e[s]>0$. la integral en el numerador se puede escribir

$$
\int_{-\infty}^{\infty} \frac{g(k, u)}{k u+\beta-i \alpha} d u
$$

donde $s=\alpha+i \beta$; por lo que la integral no es singularya que la trayectoria de integración no pasa por el polo.

Si $\operatorname{Re}[s]=0$, entonces el numerador de $(A-8)$ se escribe

$$
P \int_{-\infty}^{\infty} \frac{g(k, u)}{k u+\beta} d u+\pi i g\left(k,-\frac{\beta}{k}\right),
$$

donde $P$ indica que debe tomarse la parte principal de la integral. Puesto que $g(k, u)$ no es singular en el eje real, el primer término puede ser, a lo más, logarítmicamente infinito debido a la presencia del denominador $k u+\beta$ en el primer término. Sin embargo, para distribuciones $g(\kappa, \iota)$ bien comportadas, ese peligro no existe puesto que $g(k, u= \pm \infty)=0$. La conclusión es que el numerador de (A-8) puede contribuir sólamente con polos tales que $\operatorname{Re}\left[s_{1}\right]<0$, lo cual da soluciones $(A-10)$ que decaen con el tiempo.

En vista de que el denominador de (A-8) depende de la distribución a orden cero y no de los detalles de la perturbación inicial, los polos del denominador de (A-8) constituyen las "frecuencias naturales" del sistema. Por esta razón a la expresión

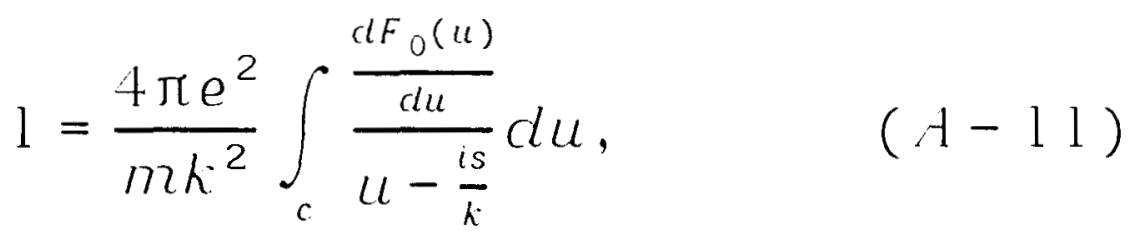

se le denomina la ecuación de dispersión de ondas electrostáticas de pequeña amplitud en el plasma. 


\section{REFERENCIAS}

1.- $\quad$ R. B. Leighton, Principles of Modern Physics. McGraw-Hill, New York 1959.

2.- $\quad$ F. Casco Sánchez y M. López Villaseñor, Análisis Espectral. UAM-I, 1993.

3.- W. Voigt and S. B. Bayer, Akad. Wiss. 603-20 (1912).

4.- $\quad$ B. D. Fried and S. D. Conte, The Plasma Dispersion Function, Academic Press, New York, 1961.

5.- M. Abramowitz and I. A. Stegun. Handbook of Mathematical Functions. Dover, 1965 , p. 297.

6.- H. Jiménez-Dominguez, A. Cabral-Prieto, L. Meléndez-Lugo, R. López-Callejas, S. Colunga-Sánchez, R. Valencia-Alvarado, S. Cruz-Jiménez, E. Gaytán-Gallardo and E. CHávez-Alarcón.

International Conference on Plasma Physics. Joint Conference of the 9th Kiev International Conference on Plasma Theory and the 9 th International Conference on Wave and Instabilities in Plasmas combined with the 19th EPS Conference on Controlled Fusion and Plasma Physics.

Innsbruck, 29 june - 3 July, 1992.

Editors: W. Freysinger, K. Lackner, R. Schrittwieser and W. Lindincer.

Vol. 16C Part III, p III-1947.

7.- H. Jiménez-Domínguez, S. Cruz-Jiménez and A, Cabral-Prieto, Spectrochim. Acta B51, 165 (1996).

8.- H. Jiménez-Domínguez, S. Cruz-Jiménez and A. Cabral-Prieto Appl. Spectrosc. $51(1997) 37$.

9.- D. W. Posener, Astr. J. Phys. 12, 184 (1959).

10.- H. S. Carlslaw and J. C. Jaeger. Operational Methods in Applied Mathematics. Dover, 1963, p. 252.

11.- L. I. Schiff, Quantum Mechanics, McGraw-Hill, third edition, pág. 415. 
12.- J. Ramirez-Torres, J. López-Monroy, A. Cabral-Prieto y H. Jiménez-Domínguez, Rev. Mex. Fis. 38, Supl. 1, 271, 1992.

13.- G. Arfken, Mathematical Methods for Physicists, 3d Edition, Academic Press, 1985, p-811.

14.- A. Cabral-Prieto, H. Jiménez-Domínguez, L. González-Tovany, S. Galindo and M. Torres-Valderrama. J. Magn. Reson. 96, 473 (1992).

15.- H. M. Nussenzveig, Causality and Dispersion Relations. Academic Press 1972. Vol. 95 in Mathematics and Engineering Series.

16.- I. S. Gradshteyn and I. M. Ryzhik. Tables of Integrals, Series and Products. Academic Press, 1965. p. 472

17.- A. G. Marshall, Editor. Fourier, Hadamard and Hilbert Transforms in Chemistry. Plenum Press, 1982, P. 108.

18.- A. Erdélyi, Editor. Tables of Integral Transforms Vol II. McGraw-Hill, 1954. p 253.

19.- H. Jiménez-Domínguez, H. Flores-LLamas, A. Cabral-Prieto and A. Bravo-Ortega. Nucl. Instr. and Meth. A278, 625 (1989).

20.- W. Gautschi, SIAM J. Numer. Anal. 7 (1970) 187.

21.- A. Simon, Linear Oscillations in a Collisionless Plasma. In: Plasma Physics, edited by OIEA, 1965.

22.- A. A. Vlasov, J. Phys. USSR, 9, 25 (1945).

23.- L. Landau, J. Phys. USSR. 10, 25 (1946).

24.- D. R. Nicholson, Introduction to Plasama Theory, John Wiley, 1983.

25.- A. Cabral-Prieto, H. Jiménez-Dominguez, L. González-Tovany, S. Galindo, H. Flores-LLamas and M. Torres-Valderrama. J. Magn. Reson. 89, 568 (1990).

26.- H. Jiménez-Domínguez and A. Cabral-Prieto. J. Magn. Reson. 93, 178 (1991).

27.- P. C. Clemmow and J. P. Dougherty. Electrodynamics of Particles and Plasmas, Addison-Wesley, 1969, p. 255.

28.- R. de L. Krönig, J. Opt. Soc. Amer. Rev. Sci. Instrum. 12, 547 (1926). 
29.- H. A. Kramers, Atti. Congr. Int. Fis. Como 2, 545 (1927).

30.- B. D. Fried, C. L. Hedrick and J. McCune. Phys. Fluids. 11, 249 (1968).

31.- P. Martin and M. A. González. Phys. Fluids. 22, 1413 (1979).

32.- P. A. Robinson and D. L. Newman. J. Plasma Phys. 40, 553 (1988).

33.- $H$. Flores-LLamas, A. Cabral-Prieto, $H$. Jiménez-Dominguez and $M$. Torres-Valderrama. Nuc. Instr. Meth. A300, 159 (1991).

34.- R. N. Bracewell. The Fourier Transform and its Applications. McGraw-Hill, 2nd. Edition, 1986, p-418.

35.- H. W. Wyld. Mathematical Methods for Physics. Benjamín, 1976.

36.- J. D. Jackson. Classical Electrodynamics. John Wiley, Second Edition, 1975.

37.- Ben Yu-Kuang Hu. Am. J. Phys. 57, 821, 1989.

38.- A. G. Marshall and D. C. Roe. Anal. Chem. 50, 756 (1978).

39.- D. C. Roe and A. G. Marshall. Anal. Chem. 50, 764 (1978).

40.- F. G. Herring, A. G. Marshall, P. S. Phillips and D. G. Roe. J. Magn. Reson. 37, 293 (1980).

41.- W. Magnus, F. Oberhettinger and F. G. Tricomi. "Tables of Integral Transforms", Volume II, A. Erdelyi Editor, p 243, McGraw-Hill, New York, 1954.

42.- A. Losev, Appl. Spectrosc. 48, 1289 (1994).

43.- E. Camps, O. Olea, C. Gutiérrez-Tapia and M. Villagrán, Rev. Sci. Instrum. 66, 3219 (1995).

44.- A. R. Striganov and N. S. Sventitskii, Tables of Spectral Lines of Neutral and Ionized Atoms, IFI/Plenum New York, 1968, p 78.

45.- Charlotte E. Moore, Atomic Levels Vol. I, United States Department of Commerce, National Bureau of Standards. Circular 467, issued 15 June 1949, p. 5.

46.- R. S. Huddlestone and S. L. Leonard (eds). Plasma Diagnostic Techniques, Academic Press, 1965, p. 265.

47.- H. R. Griem, Plasma Spectroscopy, McGraw Hill, New York, 1964 p. 101. 
48.- F. James and M. Roos, Comput. Phys. Commun. 10, 343 (1975).

49.- J. Hopwood and J. Asmussen, Appl. Phys. Lett. 58, 2473 (1991).

50.- J. Talpe and L. Van Gerven, Phys. Rev. 145, 718 (1966).

51.- Alan G. Marshall and D. Christopher Roe, Anal. Chem. 50, 756 (1978).

52.- D. Christopher Roe and Alan G. Marshall Anal Chem. 50, 764 (1978).

53.- Alan G. Marshall, editor, Fourier, Hadamard and Hilbert Transforms in Chemistry. Plenum Press, New York, 1982, p 99.

54.- M. Miglierini and J. Sitek, Phys. Stat. Sol. a93, 627 (1986).

55.- M. Miglierini, Nuc. Instrum. Meth. B36, 475 (1989).

56.- E. C. Craig and A. G. Marshall, J. Magn. Reson. 76, 458 (1988).

57.- C. A. Hutchison Jr., J. Phys. Chem. 57, 546 (1953).

58.- R. S. Alger, "Electron Paramagnetic Resonance: Techniques and Applications", Interscience, New York, 1968, p 304.

59.- C. J. Batty, S. D. Hoath and B. L. Roberts, Nucl. Instrum. Meth. 137, 179 (1976).

60.- F. Bloch, Phys Rev. 70, 37 (1946).

61.- G. E. Pake, Am. J. Phys. 18, 438 (1950).

62.- A. Abragam and B. Bleaney, "Electron Paramagnetic Resonance of Transition lons", Dover Publications, New York 1970. p-116.

63.- H.Jiménez Domínguez, S. Cruz Jiménez and A. Cabral-Prieto, Por publicarse en la revista Spectrochimica. Acta part B Atomic Spectroscopy en (1997). 